\title{
Dynamical and current-induced Dzyaloshinskii-Moriya interaction: Role for damping, gyromagnetism, and current-induced torques in noncollinear magnets
}

\author{
Frank Freimuth ${ }^{1,2}$ * Stefan Blügel ${ }^{1}$, and Yuriy Mokrousov ${ }^{1,2}$ \\ ${ }^{1}$ Peter Grünberg Institut and Institute for Advanced Simulation, \\ Forschungszentrum Jülich and JARA, 52425 Jülich, Germany and \\ 2 Institute of Physics, Johannes Gutenberg University Mainz, 55099 Mainz, Germany
}

\begin{abstract}
Both applied electric currents and magnetization dynamics modify the Dzyaloshinskii-Moriya interaction (DMI), which we call current-induced DMI (CIDMI) and dynamical DMI (DDMI), respectively. We report a theory of CIDMI and DDMI. The inverse of CIDMI consists in charge pumping by a time-dependent gradient of magnetization $\partial^{2} \boldsymbol{M}(\boldsymbol{r}, t) / \partial \boldsymbol{r} \partial t$, while the inverse of DDMI describes the torque generated by $\partial^{2} \boldsymbol{M}(\boldsymbol{r}, t) / \partial \boldsymbol{r} \partial t$. In noncollinear magnets CIDMI and DDMI depend on the local magnetization direction. The resulting spatial gradients correspond to torques that need to be included into the theories of Gilbert damping, gyromagnetism, and current-induced torques (CITs) in order to satisfy the Onsager reciprocity relations. CIDMI is related to the modification of orbital magnetism induced by magnetization dynamics, which we call dynamical orbital magnetism (DOM), and spatial gradients of DOM contribute to charge pumping. We present applications of this formalism to the CITs and to the torque-torque correlation in textured Rashba ferromagnets.
\end{abstract}

\section{INTRODUCTION}

Since the Dzyaloshinskii-Moriya interaction (DMI) controls the magnetic texture of domain walls and skyrmions, methods to tune this chiral interaction by external means have exciting prospects. Application of gate voltage [1 [3] or laser pulses [4] are promising ways to modify DMI. Additionally, theory predicts that in magnetic trilayer structures the DMI in the top magnetic layer can be controlled by the magnetization direction in the bottom magnetic layer [5]. Moreover, methods to generate spin currents may be used to induce DMI, which is predicted by the relations between the two [6, 7]. Recent experiments show that also electric currents modify DMI in metallic magnets, which leads to large changes in the domain-wall velocity $[8,9]$. However, a rigorous theoretical formalism for the investigation of current-induced DMI (CIDMI) in metallic magnets has been lacking so far, and the development of such a formalism is one goal of this paper.

Recently, a Berry phase theory of DMI [6, 10, 11] has been developed, which formally resembles the modern theory of orbital magnetization [12 14]. Orbital magnetism is modified by the application of an electric field, which is known as the orbital magnetoelectric response [15]. In the case of insulators it is straightforward to derive the expressions for the magnetoelectric response directly. However, in metals it is much easier to derive expressions instead for the inverse of the magnetoelectric response, i.e., for the generation of electric currents by time-dependent magnetic fields [16]. The inverse current-induced DMI (ICIDMI) consists in charge pumping by time-dependent gradients of magnetization. Due to the analogies between orbital magnetism and the Berry phase theory of DMI one may expect that in metals it is convenient to obtain expressions for ICIDMI, which can then be used to describe the CIDMI by exploiting the reciprocity between CIDMI and ICIDMI. We will show in this paper that this is indeed the case.

In noncentrosymmetric ferromagnets spin-orbit interaction (SOI) generates torques on the magnetization the so-called spin-orbit torques (SOTs) - when an electric current is applied [17]. The Berry phase theory of DMI [6, 10, 11] establishes a relation to SOTs. The formal analogies between orbital magnetism and DMI have been shown to be a very useful guiding principle in the development of the theory of SOTs driven by heat currents [18]. In particular, it is fruitful to consider the DMI coefficients as a spiralization, which is formally analogous to magnetization. In the theory of thermoelectric effects in magnetic systems the curl of magnetization describes a bound current, which cannot be measured in transport experiments and needs to be subtracted from the Kubo linear response in order to obtain the measurable current [19 21]. Similarly, in the theory of the thermal spin-orbit torque spatial gradients of the DMI spiralization, which result from the temperature gradient together with the temperature dependence of DMI, need to be subtracted in order to obtain the measurable torque and to satisfy a Mott-like relation [10, 18]. In noncollinear magnets the question arises whether gradients of the spiralization that are due to the magnetic texture correspond to torques like those from thermal gradients. We will show that indeed the spatial gradients of CIDMI need to be included into the theory of current-induced torques (CITs) in noncollinear magnets in order to satisfy the Onsager reciprocity relations 22.

When the system is driven out of equilibrium by magnetization dynamics rather than electric current one may expect DMI to be modified as well. The inverse effect of this dynamical DMI (DDMI) consists in the generation of torques by time-dependent magnetization gradients. In noncollinear magnets the DDMI spiralization varies in space. We will show that the resulting gradient cor- 
responds to a torque that needs to be considered in the theory of Gilbert damping and gyromagnetism in noncollinear magnets.

This paper is structured as follows. In section $\amalg A$ we give an overview of CIT in noncollinear magnets and introduce the notation. In section IIB we describe the formalism used to calculate the response of electric current to time-dependent magnetization gradients. In section IC we show that current-induced DMI (CIDMI) and electric current driven by time-dependent magnetization gradients are reciprocal effects. This allows us to obtain an expression for CIDMI based on the formalism of section IIB. In section IID we discuss that timedependent magnetization gradients generate additionally torques on the magnetization and show that the inverse effect consists in the modification of DMI by magnetization dynamics, which we call dynamical DMI (DDMI). In section IIE we demonstrate that magnetization dynamics induces orbital magnetism, which we call dynamical orbital magnetism (DOM) and show that DOM is related to CIDMI. In section IF we explain how the spatial gradients of CIDMI and DOM contribute to the direct and to the inverse CIT, respectively. In section IG we discuss how the spatial gradients of DDMI contribute to the torque-torque correlation. In section IIH we complete the formalism used to calculate the CIT in noncollinear magnets by adding the chiral contribution of the torquevelocity correlation. In section III we finalize the theory of the inverse CIT by adding the chiral contribution of the velocity-torque correlation. In section IIJ we finish the computational formalism of gyromagnetism and damping by adding the chiral contribution of the torquetorque correlation and the response of the torque to the time-dependent magnetization gradients. In section III we discuss the symmetry properties of the response to time-dependent magnetization gradients. In section IV A we present the results for the chiral contributions to the direct and the inverse CIT in the Rashba model and show that both the perturbation by the time-dependent magnetization gradient and the spatial gradients of CIDMI and DOM need to be included to ensure that they are reciprocal. In section IVB we present the results for the chiral contribution to the torque-torque correlation in the Rashba model and show that both the perturbation by the time-dependent magnetization gradient and the spatial gradients of DDMI need to be included to ensure that it satisfies the Onsager symmetry relations. This paper ends with a summary in section $\mathrm{V}$.

\section{FORMALISM}

\section{A. Direct and inverse current-induced torques in noncollinear magnets}

Even in collinear magnets the application of an electric field $\boldsymbol{E}$ generates a torque $\boldsymbol{T}^{\mathrm{CIT} 1}$ on the magnetization when inversion symmetry is broken [17, 23]:

$$
T_{i}^{\mathrm{CIT} 1}=\sum_{j} t_{i j}(\hat{\boldsymbol{M}}) E_{j}
$$

where $t_{i j}(\hat{M})$ is the torkance tensor, which depends on the magnetization direction $\hat{M}$. This torque is called spin-orbit torque (SOT), but we denote it here CIT1, because it is one contribution to the current-induced torques (CITs) in noncollinear magnets. Inversely, magnetization dynamics pumps a charge current $\boldsymbol{J}^{\text {ICIT1 ac- }}$ cording to [24]

$$
J_{i}^{\mathrm{ICIT} 1}=\sum_{j} t_{j i}(-\hat{\boldsymbol{M}}) \hat{\boldsymbol{e}}_{j} \cdot\left[\hat{\boldsymbol{M}} \times \frac{\partial \hat{\boldsymbol{M}}}{\partial t}\right]
$$

where $\hat{\boldsymbol{e}}_{j}$ is a unit vector that points into the $j$-th spatial direction. Generally, $\boldsymbol{J}^{\text {ICIT1 }}$ can be explained by the inverse spin-orbit torque [24] or the magnonic charge pumping 25]. We denote it here by ICIT1, because it is one contribution to the inverse CIT in noncollinear magnets. In the special case of magnetic bilayers one important mechanism responsible for $\boldsymbol{J}^{\text {ICIT1 }}$ arises from the combination of spin pumping and the inverse spin Hall effect [26, 27].

In noncollinear magnets there is a second contribution to the CIT, which is proportional to the spatial derivatives of magnetization [28]:

$$
T_{i}^{\mathrm{CIT} 2}=\sum_{j k l} \chi_{i j k l}^{\mathrm{CIT} 2} E_{j} \hat{\boldsymbol{e}}_{k} \cdot\left[\hat{\boldsymbol{M}} \times \frac{\partial \hat{\boldsymbol{M}}}{\partial r_{l}}\right] .
$$

The description of noncollinearity by the derivatives $\partial \hat{\boldsymbol{M}} / \partial r_{l}$ is only applicable when the magnetization direction changes slowly in space like in magnetic skyrmions with large radius and in wide magnetic domain walls. In order to treat noncollinear magnets such as $\mathrm{Mn}_{3} \mathrm{Sn}$ [29], where the magnetization direction varies strongly on the scale of one unit cell, Eq. (3) needs to be modified, which is beyond the scope of the present paper. The adiabatic and the non-adiabatic [30] spin transfer torques are two important contributions to $\chi_{i j k l}^{\mathrm{CIT} 2}$, but the interplay between broken inversion symmetry, SOI, and noncollinearity can lead to a large number of additional mechanisms 22, 31]. Similarly, the current pumped by magnetization dynamics contains a contribution that is proportional to the spatial derivatives of magnetiza- 
tion [22, 32, 33]:

$$
J_{i}^{\mathrm{ICIT} 2}=\sum_{j k l} \chi_{i j k l}^{\mathrm{ICIT} 2} \hat{\boldsymbol{e}}_{j} \cdot\left[\hat{\boldsymbol{M}} \times \frac{\partial \hat{\boldsymbol{M}}}{\partial t}\right] \hat{\boldsymbol{e}}_{k} \cdot\left[\hat{\boldsymbol{M}} \times \frac{\partial \hat{\boldsymbol{M}}}{\partial r_{l}}\right] .
$$

$T_{i}^{\mathrm{CIT} 2}$ and $J_{i}^{\mathrm{ICIT} 2}$ can be considered as chiral contributions to the CIT and to the ICIT, respectively, because they distinguish between left- and right-handed spin spirals. Due to the reciprocity between direct and inverse CIT 22, 24] the coefficients $\chi_{i j k l}^{\mathrm{ICIT} 2}$ and $\chi_{j i k l}^{\mathrm{CIT} 2}$ are related according to

$$
\chi_{i j k l}^{\mathrm{ICIT2} 2}(\hat{\boldsymbol{M}})=\chi_{j i k l}^{\mathrm{CIT} 2}(-\hat{\boldsymbol{M}}) .
$$

\section{B. Response of electric current to time-dependent magnetization gradients}

In order to compute $\boldsymbol{J}^{\text {ICIT2 }}$ based on the Kubo linear response formalism it is necessary to split it into two contributions, $\boldsymbol{J}^{\text {ICIT2a }}$ and $\boldsymbol{J}^{\text {ICIT2b }}$. While $\boldsymbol{J}^{\text {ICIT2a }}$ is obtained as linear response to the perturbation by a time-dependent magnetization gradient in a collinear ferromagnet, $\boldsymbol{J}^{\text {ICIT2b }}$ is obtained as linear response to the perturbation by magnetization dynamics in a noncollinear ferromagnet. Therefore, as will become clear below, $\boldsymbol{J}^{\text {ICIT2a }}$ can be expressed by a correlation function of two operators, because it describes the response of the current to a time-dependent magnetization gradient: A time-dependent magnetization gradient is a single perturbation, which is described by a single perturbing operator. In contrast, $\boldsymbol{J}^{\text {ICIT2b }}$ involves the correlation of three operators, because it describes the response of the current to magnetization dynamics in the presence of perturbation by noncollinearity. These are two perturbations: One perturbation by the magnetization dynamics, and a second perturbation to describe the noncollinearity. In the Kubo formalism the expressions for the response one the one hand to a time-dependent magnetization gradient, which is described by a single perturbing operator, and the response on the other hand to a time-dependent magnetization in the presence of a magnetization gradient, which is described by two perturbing operators, are different. Therefore, we split $\boldsymbol{J}^{\text {ICIT2 }}$ into these two contributions, which we call $\boldsymbol{J}^{\mathrm{ICIT2a}}$ and $\boldsymbol{J}^{\mathrm{ICIT2b}}$. In the remainder of this section we discuss the calculation of the contribution $\boldsymbol{J}^{\text {ICIT2a }}$. The contribution $\boldsymbol{J}^{\text {ICIT2b }}$ is discussed in section III below.

$\boldsymbol{J}^{\text {ICIT2a }}$ is determined by the second derivative of magnetization with respect to time and space variables and can be written as

$$
J_{i}^{\mathrm{ICIT} 2 \mathrm{a}}=\sum_{j k} \chi_{i j k}^{\mathrm{ICIT2a}} \frac{\partial^{2} \hat{M}_{j}}{\partial r_{k} \partial t} .
$$

A nonzero second derivative $\frac{\partial^{2} \hat{M}_{j}}{\partial r_{k} \partial t}$ is what we refer to as a time-dependent magnetization gradient. We will show be- low that in special cases $\frac{\partial^{2} \hat{M}_{j}}{\partial r_{k} \partial t}$ can be expressed in terms of the products $\frac{\partial \hat{M}_{l}}{\partial r_{k}} \frac{\partial \hat{M}_{l}}{\partial t}$, which will allow us to rewrite $J_{i}^{\mathrm{ICIT2a}}$ in the form of Eq. (4) in the cases relevant for the chiral ICIT. However, as will become clear below, Eq. (6) is the most general expression for the response to time-dependent magnetization gradients, and it cannot generally be rewritten in the form of Eq. (44): This is only possible when it describes a contribution to the chiral ICIT.

$\boldsymbol{J}^{\text {ICIT2a }}$ occurs in two different situations, which need to be distinguished. In one case the magnetization gradient varies in time like $\sin (\omega t)$ everywhere in space. An example is

$$
\hat{\boldsymbol{M}}(\boldsymbol{r}, t)=\left(\begin{array}{c}
\eta \sin (\boldsymbol{q} \cdot \boldsymbol{r}) \sin (\omega t) \\
0 \\
1
\end{array}\right),
$$

where $\eta$ is the amplitude and the derivatives at $t=0$ and $\boldsymbol{r}=0$ are

$$
\left.\frac{\partial \hat{\boldsymbol{M}}(\boldsymbol{r}, t)}{\partial r_{i}}\right|_{\boldsymbol{r}=t=0}=\left.\frac{\partial \hat{\boldsymbol{M}}(\boldsymbol{r}, t)}{\partial t}\right|_{\boldsymbol{r}=t=0}=0
$$

and

$$
\left.\frac{\partial^{2} \hat{\boldsymbol{M}}(\boldsymbol{r}, t)}{\partial r_{i} \partial t}\right|_{\boldsymbol{r}=t=0}=\left(\begin{array}{c}
\eta q_{i} \omega \\
0 \\
0
\end{array}\right) .
$$

In the other case the magnetic texture varies like a propagating wave, i.e., proportional to $\sin (\boldsymbol{q} \cdot \boldsymbol{r}-\omega t)$. An example is given by

$$
\hat{\boldsymbol{M}}(\boldsymbol{r}, t)=\left(\begin{array}{c}
\eta \sin (\boldsymbol{q} \cdot \boldsymbol{r}-\omega t) \\
0 \\
1-\frac{\eta^{2}}{2} \sin ^{2}(\boldsymbol{q} \cdot \boldsymbol{r}-\omega t)
\end{array}\right),
$$

where the derivatives at $t=0$ and $\boldsymbol{r}=0$ are

$$
\begin{aligned}
& \left.\frac{\partial \hat{\boldsymbol{M}}(\boldsymbol{r}, t)}{\partial r_{i}}\right|_{\boldsymbol{r}=t=0}=\left(\begin{array}{c}
\eta q_{i} \\
0 \\
0
\end{array}\right), \\
& \left.\frac{\partial \hat{\boldsymbol{M}}(\boldsymbol{r}, t)}{\partial t}\right|_{\boldsymbol{r}=t=0}=\left(\begin{array}{c}
-\eta \omega \\
0 \\
0
\end{array}\right)
\end{aligned}
$$

and

$$
\left.\frac{\partial^{2} \hat{\boldsymbol{M}}(\boldsymbol{r}, t)}{\partial r_{i} \partial t}\right|_{\boldsymbol{r}=t=0}=\left(\begin{array}{c}
0 \\
0 \\
\eta^{2} q_{i} \omega
\end{array}\right) .
$$

In the latter example, Eq. (10), the second derivative, Eq. (13), is along the magnetization $\hat{M}(\boldsymbol{r}=0, t=0)$, while in the former example, Eq. (7), the second derivative, Eq. (9), is perpendicular to the magnetization when $\boldsymbol{r}=0$ and $t=0$. 
We assume that the Hamiltonian is given by

$$
\begin{aligned}
H(\boldsymbol{r}, t)= & -\frac{\hbar^{2}}{2 m_{e}} \Delta+V(\boldsymbol{r})+\mu_{\mathrm{B}} \hat{\boldsymbol{M}}(\boldsymbol{r}, t) \cdot \boldsymbol{\sigma} \Omega^{\mathrm{xc}}(\boldsymbol{r})+ \\
& +\frac{1}{2 e c^{2}} \mu_{\mathrm{B}} \boldsymbol{\sigma} \cdot[\nabla V(\boldsymbol{r}) \times \boldsymbol{v}]
\end{aligned}
$$

where the first term describes the kinetic energy, the second term is a scalar potential, $\Omega^{\mathrm{xc}}(\boldsymbol{r})$ in the third term is the exchange field, and the last term describes the spinorbit interaction. Around $t=0$ and $\boldsymbol{r}=0$ we can decompose the Hamiltonian as $H(\boldsymbol{r}, t)=H_{0}+\delta H(\boldsymbol{r}, t)$, where $H_{0}$ is obtained from $H(\boldsymbol{r}, t)$ by replacing $\hat{\boldsymbol{M}}(\boldsymbol{r}, t)$ by $\hat{M}(\boldsymbol{r}=0, t=0)$ and

$$
\begin{aligned}
\delta H(\boldsymbol{r}, t) & =\frac{\partial H_{0}}{\partial \hat{M}_{x}} \eta \sin (\boldsymbol{q} \cdot \boldsymbol{r}) \sin (\omega t) \\
& =\mu_{\mathrm{B}} \Omega^{\mathrm{xc}}(\boldsymbol{r}) \sigma_{x} \eta \sin (\boldsymbol{q} \cdot \boldsymbol{r}) \sin (\omega t)
\end{aligned}
$$

in the case of the first example, Eq. (77). In the case of the second example, Eq. (10),

$$
\begin{aligned}
\delta H(\boldsymbol{r}, t) & \simeq \frac{\partial H}{\partial \hat{M}_{x}} \eta \sin (\boldsymbol{q} \cdot \boldsymbol{r}-\omega t) \\
& +\frac{\partial H}{\partial \hat{M}_{z}} \eta^{2} \sin (\boldsymbol{q} \cdot \boldsymbol{r}) \sin (\omega t),
\end{aligned}
$$

where for small $\boldsymbol{r}$ and $t$ only the second term on the righthand side contributes to $\frac{\partial^{2} H(\boldsymbol{r}, t)}{\partial r_{k} \partial t}$. We consider here only the time-dependence of the exchange field direction and ignore the time-dependence of the exchange field magnitude $\Omega^{\mathrm{xc}}(\boldsymbol{r})$ that is induced by the time-dependence of the exchange field direction. While the variation of the exchange field magnitude drives currents and torques as well, as shown in Ref. [34], the variation of the exchange field magnitude is a small response and therefore these secondary responses are suppressed in magnitude when compared to the direct primary responses of the current and torque to the variation in the exchange field direction. We will use the perturbations Eq. (15) and Eq. (16) in order to compute the response of current and torque within the Kubo response formalism. An alternative approach for the calculation of the response to timedependent fields is variational linear-response, which has been applied to the spin susceptibility by Savrasov [35].

The perturbation by the time-dependent gradient can be written as

$$
\delta H=\frac{\partial H}{\partial \hat{M}} \cdot \frac{\partial^{2} \hat{M}}{\partial r_{i} \partial t} \frac{\sin \left(q_{i} r_{i}\right)}{q_{i}} \frac{\sin (\omega t)}{\omega},
$$

which turns into Eq. (15) when Eq. (9) is inserted. When Eq. (13) is inserted it turns into the second term in Eq. (16).

In Appendix $\mathrm{A}$ we derive the linear response to perturbations of the type of Eq. (17) and show that the corresponding coefficient $\chi_{i j k}^{\mathrm{ICIT2a}}$ in Eq. (6) can be expressed as

$$
\begin{aligned}
\chi_{i j k}^{\mathrm{ICIT} 2 \mathrm{a}}= & \frac{i e}{4 \pi \hbar^{2}} \int \frac{\mathrm{d}^{d} k}{(2 \pi)^{d}} \int d \mathcal{E} f(\mathcal{E}) \operatorname{Tr}[ \\
& v_{i} R v_{k} R R \mathcal{O}_{j} R+v_{i} R R v_{k} R \mathcal{O}_{j} R+ \\
& -v_{i} R R \mathcal{O}_{j} R v_{k} R-v_{i} R v_{k} R \mathcal{O}_{j} A A \\
& +v_{i} R \mathcal{O}_{j} A v_{k} A A+v_{i} R \mathcal{O}_{j} A A v_{k} A \\
& -v_{i} R v_{k} R R \mathcal{O}_{j} A-v_{i} R R v_{k} R \mathcal{O}_{j} A \\
& +v_{i} R R \mathcal{O}_{j} A v_{k} A+v_{i} A v_{k} A \mathcal{O}_{j} A A \\
& \left.-v_{i} A \mathcal{O}_{j} A v_{k} A A-v_{i} A \mathcal{O}_{j} A A v_{k} A\right]
\end{aligned}
$$

where $R=G_{\boldsymbol{k}}^{\mathrm{R}}(\mathcal{E})$ and $A=G_{\boldsymbol{k}}^{\mathrm{A}}(\mathcal{E})$ are shorthands for the retarded and advanced Green's functions, respectively, and $\mathcal{O}_{j}=\partial H / \partial \hat{M}_{j}$. $e>0$ is the positive elementary charge.

In the case of the perturbation of the type Eq. (7) the second derivative $\frac{\partial^{2} \hat{M}}{\partial r_{i} \partial t}$ is perpendicular to $\boldsymbol{M}$. In this case it is convenient to rewrite Eq. (6) as

$$
J_{i}^{\mathrm{ICIT} 2 \mathrm{a}}=\sum_{j k} \chi_{i j k}^{\mathrm{ICIDMI}} \hat{\boldsymbol{e}}_{j} \cdot\left[\hat{\boldsymbol{M}} \times \frac{\partial^{2} \hat{\boldsymbol{M}}}{\partial r_{k} \partial t}\right]
$$

where the coefficients $\chi_{i j k}^{\mathrm{ICIDMI}}$ are given by

$$
\begin{aligned}
\chi_{i j k}^{\mathrm{ICIDMI}}= & \frac{i e}{4 \pi \hbar^{2}} \int \frac{\mathrm{d}^{d} k}{(2 \pi)^{d}} \int d \mathcal{E} f(\mathcal{E}) \operatorname{Tr}[ \\
& v_{i} R v_{k} R R \mathcal{T}_{j} R+v_{i} R R v_{k} R \mathcal{T}_{j} R+ \\
& -v_{i} R R \mathcal{T}_{j} R v_{k} R-v_{i} R v_{k} R \mathcal{T}_{j} A A \\
& +v_{i} R \mathcal{T}_{j} A v_{k} A A+v_{i} R \mathcal{T}_{j} A A v_{k} A \\
& -v_{i} R v_{k} R R \mathcal{T}_{j} A-v_{i} R R v_{k} R \mathcal{T}_{j} A \\
& +v_{i} R R \mathcal{T}_{j} A v_{k} A+v_{i} A v_{k} A \mathcal{T}_{j} A A \\
& \left.-v_{i} A \mathcal{T}_{j} A v_{k} A A-v_{i} A \mathcal{T}_{j} A A v_{k} A\right]
\end{aligned}
$$

and

$$
\mathcal{T}=\hat{M} \times \frac{\partial H}{\partial \hat{M}}
$$

is the torque operator. In Sec. IC we will explain that $\chi_{i j k}^{\text {ICIDMI }}$ describes the inverse of current-induced DMI (ICIDMI).

In the case of the perturbation of the type of Eq. (10) the second derivative $\frac{\partial^{2} \hat{M}_{j}}{\partial r_{k} \partial t}$ may be rewritten as product of the first derivatives $\frac{\partial \hat{M}_{l}}{\partial t}$ and $\frac{\partial \hat{M}_{l}}{\partial r_{k}}$. This may be seen 
as follows:

$$
\begin{aligned}
& \frac{\partial H}{\partial \hat{\boldsymbol{M}}} \cdot \frac{\partial^{2} \hat{\boldsymbol{M}}}{\partial r_{i} \partial t}=\frac{\partial^{2} H}{\partial t \partial r_{i}}= \\
& =\frac{\partial}{\partial t}\left[\left(\hat{\boldsymbol{M}} \times \frac{\partial H}{\partial \hat{M}}\right) \cdot\left(\hat{\boldsymbol{M}} \times \frac{\partial \hat{\boldsymbol{M}}}{\partial r_{i}}\right)\right]= \\
& =\left[\left(\frac{\partial \hat{\boldsymbol{M}}}{\partial t} \times \frac{\partial H}{\partial \hat{\boldsymbol{M}}}\right) \cdot\left(\hat{\boldsymbol{M}} \times \frac{\partial \hat{\boldsymbol{M}}}{\partial r_{i}}\right)\right]= \\
& =\left[\left(\left(\hat{\boldsymbol{M}} \times \frac{\partial \hat{\boldsymbol{M}}}{\partial t}\right) \times \hat{\boldsymbol{M}}\right) \times \frac{\partial H}{\partial \hat{\boldsymbol{M}}}\right] \cdot\left[\hat{\boldsymbol{M}} \times \frac{\partial \hat{\boldsymbol{M}}}{\partial r_{i}}\right]= \\
& =-\left[\hat{\boldsymbol{M}} \times \frac{\partial \hat{\boldsymbol{M}}}{\partial t}\right] \cdot\left[\hat{\boldsymbol{M}} \times \frac{\partial \hat{\boldsymbol{M}}}{\partial r_{i}}\right]\left[\hat{\boldsymbol{M}} \cdot \frac{\partial H}{\partial \hat{\boldsymbol{M}}}\right]= \\
& =-\frac{\partial \hat{\boldsymbol{M}}}{\partial t} \cdot \frac{\partial \hat{\boldsymbol{M}}}{\partial r_{i}}\left[\hat{\boldsymbol{M}} \cdot \frac{\partial H}{\partial \hat{\boldsymbol{M}}}\right] \text {. }
\end{aligned}
$$

This expression is indeed satisfied by Eq. (11), Eq. (12) and Eq. (13):

$$
\frac{\partial \hat{\boldsymbol{M}}}{\partial r_{i}} \cdot \frac{\partial \hat{\boldsymbol{M}}}{\partial t}=-\frac{\partial^{2} \hat{\boldsymbol{M}}}{\partial r_{i} \partial t} \cdot \hat{\boldsymbol{M}}
$$

at $\boldsymbol{r}=0, t=0$. Consequently, Eq. (6) can be rewritten as

$$
\begin{aligned}
J_{i}^{\mathrm{ICIT} 2 \mathrm{a}} & =\sum_{j k} \chi_{i j k}^{\mathrm{ICIT} 2 \mathrm{a}} \frac{\partial^{2} \hat{M}_{j}}{\partial r_{k} \partial t}= \\
& =-\sum_{j k l} \chi_{i j k}^{\mathrm{ICIT} 2 \mathrm{a}} \frac{\partial \hat{M}_{l}}{\partial r_{k}} \frac{\partial \hat{M}_{l}}{\partial t}\left[1-\delta_{j l}\right] \\
& =\sum_{j k l} \chi_{i j k l}^{\mathrm{ICIT} 2 \mathrm{a}} \hat{\boldsymbol{e}}_{j} \cdot\left[\hat{\boldsymbol{M}} \times \frac{\partial \hat{\boldsymbol{M}}}{\partial t}\right] \hat{\boldsymbol{e}}_{k} \cdot\left[\hat{\boldsymbol{M}} \times \frac{\partial \hat{\boldsymbol{M}}}{\partial r_{l}}\right],
\end{aligned}
$$

where

$$
\chi_{i j k l}^{\mathrm{ICIT2a}}=-\sum_{m} \chi_{i m l}^{\mathrm{ICIT} 2 \mathrm{a}}\left[1-\delta_{j m}\right] \delta_{j k} .
$$

Thus, Eq. (24) and Eq. (25) can be used to express $J_{i}^{\mathrm{ICIT} 2 \mathrm{a}}$ in the form of Eq. (4).

\section{Direct and inverse CIDMI}

Eq. (20) describes the response of the electric current to time-dependent magnetization gradients of the type Eq. (15). The reciprocal process consists in the currentinduced modification of DMI. This can be shown by expressing the DMI coefficients as [10]

$$
\begin{aligned}
D_{i j} & =\frac{1}{V} \sum_{n} f\left(\mathcal{E}_{\boldsymbol{k} n}\right) \int d^{3} r\left(\psi_{\boldsymbol{k} n}(\boldsymbol{r})\right)^{*} \mathcal{D}_{i j} \psi_{\boldsymbol{k} n}(\boldsymbol{r}) \\
& =\frac{1}{V} \sum_{n} f\left(\mathcal{E}_{\boldsymbol{k} n}\right) \int d^{3} r\left(\psi_{\boldsymbol{k} n}(\boldsymbol{r})\right)^{*} \mathcal{T}_{i}(\boldsymbol{r}) r_{j} \psi_{\boldsymbol{k} n}(\boldsymbol{r}),
\end{aligned}
$$

where we defined the DMI-operator $\mathcal{D}_{i j}=\mathcal{T}_{i} r_{j}$. Using the Kubo formalism the current-induced modification of DMI may be written as

$$
D_{i j}^{\mathrm{CIDMI}}=\sum_{k} \chi_{k i j}^{\mathrm{CIDMI}} E_{k}
$$

with

$$
\chi_{k i j}^{\mathrm{CIDMI}}=\frac{1}{V} \lim _{\omega \rightarrow 0}\left[\frac{e}{\hbar \omega} \operatorname{Im}\left\langle\left\langle\mathcal{D}_{i j} ; v_{k}\right\rangle\right\rangle^{\mathrm{R}}(\hbar \omega)\right],
$$

where

$$
\left\langle\left\langle\mathcal{D}_{i j} ; v_{k}\right\rangle\right\rangle^{\mathrm{R}}(\hbar \omega)=-i \int_{0}^{\infty} d t e^{i \omega t}\left\langle\left[\mathcal{D}_{i j}(t), v_{k}(0)\right]_{-}\right\rangle
$$

is the Fourier transform of a retarded function and $V$ is the volume of the unit cell.

Since the position operator $r$ in the DMI operator $\mathcal{D}_{i j}=\mathcal{T}_{i} r_{j}$ is not compatible with Bloch periodic boundary conditions, we do not use Eq. (28) for numerical calculations of CIDMI. However, it is convenient to use Eq. (28) in order to demonstrate the reciprocity between direct and inverse CIDMI.

Inverse CIDMI (ICIDMI) describes the electric current that responds to the perturbation by a time-dependent magnetization gradient according to

$$
J_{k}^{\mathrm{ICIDMI}}=\sum_{i j} \chi_{k i j}^{\mathrm{ICIDMI}} \hat{\boldsymbol{e}}_{i} \cdot\left[\hat{\boldsymbol{M}} \times \frac{\partial^{2} \hat{\boldsymbol{M}}}{\partial t \partial r_{j}}\right] .
$$

The perturbation by a time-dependent magnetization gradient may be written as

$$
\begin{aligned}
\delta H & =-\sum_{j} \boldsymbol{m} \cdot \frac{\partial^{2} \hat{\boldsymbol{M}}}{\partial t \partial r_{j}} r_{j} \Omega^{\mathrm{xc}}(\boldsymbol{r}) \frac{\sin (\omega t)}{\omega}= \\
& =\sum_{j} \mathcal{T} \cdot\left[\hat{\boldsymbol{M}} \times \frac{\partial^{2} \hat{\boldsymbol{M}}}{\partial t \partial r_{j}}\right] r_{j} \frac{\sin (\omega t)}{\omega} \\
& =\sum_{i j} \mathcal{D}_{i j} \hat{\boldsymbol{e}}_{i} \cdot\left[\hat{\boldsymbol{M}} \times \frac{\partial^{2} \hat{\boldsymbol{M}}}{\partial t \partial r_{j}}\right] \frac{\sin (\omega t)}{\omega} .
\end{aligned}
$$

Consequently, the coefficient $\chi_{k i j}^{\mathrm{ICIDMI}}$ is given by

$$
\chi_{k i j}^{\mathrm{ICIDMI}}=\frac{1}{V} \lim _{\omega \rightarrow 0}\left[\frac{e}{\hbar \omega} \operatorname{Im}\left\langle\left\langle v_{k} ; \mathcal{D}_{i j}\right\rangle\right\rangle^{\mathrm{R}}(\hbar \omega)\right] .
$$

Using

$$
\left\langle\left\langle\mathcal{D}_{i j} ; v_{k}\right\rangle\right\rangle^{\mathrm{R}}(\hbar \omega, \hat{\boldsymbol{M}})=-\left\langle\left\langle v_{k} ; \mathcal{D}_{i j}\right\rangle\right\rangle^{\mathrm{R}}(\hbar \omega,-\hat{\boldsymbol{M}})
$$

we find that CIDMI and ICIDMI are related through the equations

$$
\chi_{k i j}^{\mathrm{CIDMI}}(\hat{\boldsymbol{M}})=-\chi_{k i j}^{\mathrm{ICIDMI}}(-\hat{\boldsymbol{M}}) .
$$

In order to calculate CIDMI we use Eq. (20) for ICIDMI and then use Eq. (34) to obtain CIDMI. 
The perturbation Eq. (16) describes a different kind of time-dependent magnetization gradient, for which the reciprocal effect consists in the modification of the expectation value $\left\langle\boldsymbol{\sigma} \cdot \hat{\boldsymbol{M}} r_{j}\right\rangle$. However, while the modification of $\left\langle\mathcal{T}_{i} r_{j}\right\rangle$ by an applied current can be measured [8, 9] from the change of the DMI constant $D_{i j}$, the quantity $\left\langle\boldsymbol{\sigma} \cdot \hat{\boldsymbol{M}} r_{j}\right\rangle$ has not been considered so far in ferromagnets. In noncollinear magnets the quantity $\left\langle\boldsymbol{\sigma} r_{j}\right\rangle$ can be used to define spin toroidization [36]. Therefore, while the perturbation of the type of Eq. (15) is related to CIDMI and ICIDMI, which are both accessible experimentally [8, 9], in the case of the perturbation of the type of Eq. (16) we expect that only the effect of driving current by the time-dependent magnetization gradient is easily accessible experimentally, while its inverse effect is difficult to measure.

\section{Direct and inverse dynamical DMI}

Not only applied electric currents modify DMI, but also magnetization dynamics, which we call dynamical DMI (DDMI). DDMI can be expressed as

$$
D_{i j}^{\mathrm{DDMI}}=\sum_{k} \chi_{k i j}^{\mathrm{DDMI}} \hat{\boldsymbol{e}}_{k} \cdot\left[\hat{\boldsymbol{M}} \times \frac{\partial \hat{\boldsymbol{M}}}{\partial t}\right] .
$$

In Sec. IIG we will show that the spatial gradient of DDMI contributes to damping and gyromagnetism in noncollinear magnets. The perturbation used to describe magnetization dynamics is given by [24]

$$
\delta H=\frac{\sin (\omega t)}{\omega}\left(\hat{\boldsymbol{M}} \times \frac{\partial \hat{\boldsymbol{M}}}{\partial t}\right) \cdot \mathcal{T} .
$$

Consequently, the coefficients $\chi_{k i j}^{\mathrm{DDMI}}$ may be written as

$$
\chi_{k i j}^{\mathrm{DDMI}}=-\frac{1}{V} \lim _{\omega \rightarrow 0}\left[\frac{1}{\hbar \omega} \operatorname{Im}\left\langle\left\langle\mathcal{D}_{i j} ; \mathcal{T}_{k}\right\rangle\right\rangle^{\mathrm{R}}(\hbar \omega)\right] .
$$

Since the position operator in $\mathcal{D}_{i j}$ is not compatible with Bloch periodic boundary conditions, we do not use Eq. (37) for numerical calculations of DDMI, but instead we obtain it from its inverse effect, which consists in the generation of torques on the magnetization due to timedependent magnetization gradients. These torques can be written as

$$
T_{k}^{\mathrm{IDDMI}}=\sum_{i j} \chi_{k i j}^{\mathrm{IDDMI}} \hat{\boldsymbol{e}}_{i} \cdot\left[\hat{\boldsymbol{M}} \times \frac{\partial^{2} \hat{\boldsymbol{M}}}{\partial t \partial r_{j}}\right],
$$

where the coefficients $\chi_{k i j}^{\mathrm{IDDMI}}$ are

$$
\chi_{k i j}^{\mathrm{IDDMI}}=\frac{1}{V} \lim _{\omega \rightarrow 0}\left[\frac{1}{\hbar \omega} \operatorname{Im}\left\langle\left\langle\mathcal{T}_{k} ; \mathcal{D}_{i j}\right\rangle\right\rangle^{\mathrm{R}}(\hbar \omega)\right],
$$

because the perturbation by the time-dependent gradient can be expressed in terms of $\mathcal{D}_{i j}$ according to Eq. (31) and because the torque on the magnetization is described by $-\mathcal{T}[23]$. Consequently, DDMI and IDDMI are related by

$$
\chi_{k i j}^{\mathrm{DDMI}}(\hat{\boldsymbol{M}})=-\chi_{k i j}^{\mathrm{IDDMI}}(-\hat{\boldsymbol{M}}) .
$$

For numerical calculations of IDDMI we use

$$
\begin{aligned}
\chi_{i j k}^{\mathrm{IDDMI}}= & \frac{i}{4 \pi \hbar^{2}} \int \frac{\mathrm{d}^{d} k}{(2 \pi)^{d}} \int d \mathcal{E} f(\mathcal{E}) \operatorname{Tr}[ \\
& \mathcal{T}_{i} R v_{k} R R \mathcal{T}_{j} R+\mathcal{T}_{i} R R v_{k} R \mathcal{T}_{j} R+ \\
& -\mathcal{T}_{i} R R \mathcal{T}_{j} R v_{k} R-\mathcal{T}_{i} R v_{k} R \mathcal{T}_{j} A A \\
& +\mathcal{T}_{i} R \mathcal{T}_{j} A v_{k} A A+\mathcal{T}_{i} R \mathcal{T}_{j} A A v_{k} A \\
& -\mathcal{T}_{i} R v_{k} R R \mathcal{T}_{j} A-\mathcal{T}_{i} R R v_{k} R \mathcal{T}_{j} A \\
& +\mathcal{T}_{i} R R \mathcal{T}_{j} A v_{k} A+\mathcal{T}_{i} A v_{k} A \mathcal{T}_{j} A A \\
& \left.-\mathcal{T}_{i} A \mathcal{T}_{j} A v_{k} A A-\mathcal{T}_{i} A \mathcal{T}_{j} A A v_{k} A\right]
\end{aligned}
$$

which is derived in Appendix $\mathrm{A}$. In order to obtain DDMI we calculate IDDMI from Eq. (41) and use the reciprocity relation Eq. (40).

Eq. (38) is valid for time-dependent magnetization gradients that lead to perturbations of the type of Eq. (15). Perturbations of the second type, Eq. (16), will induce torques on the magnetization as well. However, the inverse effect is difficult to measure in that case, because it corresponds to the modification of the expectation value $\left\langle\boldsymbol{\sigma} \cdot \hat{\boldsymbol{M}} r_{j}\right\rangle$ by magnetization dynamics. Therefore, while in the case of Eq. (15) both direct and inverse response are expected to be measurable and correspond to IDDMI and DDMI, respectively, we expect that in the case of Eq. (16) only the direct effect, i.e., the response of the torque to the perturbation, is easy to observe.

\section{E. Dynamical orbital magnetism (DOM)}

Magnetization dynamics does not only induce DMI, but also orbital magnetism, which we call dynamical orbital magnetism (DOM). It can be written as

$$
M_{i j}^{\mathrm{DOM}}=\sum_{k} \chi_{k i j}^{\mathrm{DOM}} \hat{\boldsymbol{e}}_{k} \cdot\left[\hat{\boldsymbol{M}} \times \frac{\partial \hat{\boldsymbol{M}}}{\partial t}\right],
$$

where we introduced the notation

$$
M_{i j}^{\mathrm{DOM}}=\frac{e}{V}\left\langle v_{i} r_{j}\right\rangle^{\mathrm{DOM}},
$$

which defines a generalized orbital magnetization, such that

$$
M_{i}^{\mathrm{DOM}}=\frac{1}{2} \sum_{j k} \epsilon_{i j k} M_{j k}^{\mathrm{DOM}}
$$


corresponds to the usual definition of orbital magnetization. The coefficients $\chi_{k i j}^{\mathrm{DOM}}$ are given by

$$
\chi_{k i j}^{\mathrm{DOM}}=-\frac{1}{V} \lim _{\omega \rightarrow 0}\left[\frac{e}{\hbar \omega} \operatorname{Im}\left\langle\left\langle v_{i} r_{j} ; \mathcal{T}_{k}\right\rangle\right\rangle^{\mathrm{R}}(\hbar \omega)\right],
$$

because the perturbation by magnetization dynamics is described by Eq. (36). We will discuss in Sec. IF that the spatial gradient of DOM contributes to the inverse CIT. Additionally, we will show below that DOM and CIDMI are related to each other.

In order to obtain an expression for DOM it is convenient to consider the inverse effect, i.e., the generation of a torque by the application of a time-dependent magnetic field $\boldsymbol{B}(t)$ that acts only on the orbital degrees of freedom of the electrons and not on their spins. This torque can be written as

$$
\mathcal{T}_{k}^{\mathrm{IDOM}}=\frac{1}{2} \sum_{i j l} \chi_{k i j}^{\mathrm{IDOM}} \epsilon_{i j l} \frac{\partial B_{l}}{\partial t},
$$

where

$$
\chi_{k i j}^{\mathrm{IDOM}}=-\frac{1}{V} \lim _{\omega \rightarrow 0}\left[\frac{e}{\hbar \omega} \operatorname{Im}\left\langle\left\langle\mathcal{T}_{k} ; v_{i} r_{j}\right\rangle\right\rangle^{\mathrm{R}}(\hbar \omega)\right],
$$

because the perturbation by the time-dependent magnetic field is given by

$$
\delta H=-\frac{e}{2} \sum_{i j k} \epsilon_{i j k} v_{i} r_{j} \frac{\partial B_{k}}{\partial t} \frac{\sin (\omega t)}{\omega} .
$$

Therefore, the coefficients of DOM and IDOM are related by

$$
\chi_{k i j}^{\mathrm{DOM}}(\hat{\boldsymbol{M}})=-\chi_{k i j}^{\mathrm{IDOM}}(-\hat{\boldsymbol{M}})
$$

In Appendix $\mathrm{A}$ we show that the coefficient $\chi_{i j k}^{\mathrm{IDOM}}$ can be expressed as

$$
\begin{aligned}
\chi_{i j k}^{\mathrm{IDOM}}= & \frac{-i e}{4 \pi \hbar^{2}} \int \frac{\mathrm{d}^{d} k}{(2 \pi)^{d}} \int d \mathcal{E} f(\mathcal{E}) \operatorname{Tr}[ \\
& \mathcal{T}_{i} R v_{k} R R v_{j} R+\mathcal{T}_{i} R R v_{k} R v_{j} R+ \\
- & \mathcal{T}_{i} R R v_{j} R v_{k} R-\mathcal{T}_{i} R v_{k} R v_{j} A A \\
+ & \mathcal{T}_{i} R v_{j} A v_{k} A A+\mathcal{T}_{i} R v_{j} A A v_{k} A \\
- & \mathcal{T}_{i} R v_{k} R R v_{j} A-\mathcal{T}_{i} R R v_{k} R v_{j} A \\
+ & \mathcal{T}_{i} R R v_{j} A v_{k} A+\mathcal{T}_{i} A v_{k} A v_{j} A A \\
& \left.-\mathcal{T}_{i} A v_{j} A v_{k} A A-\mathcal{T}_{i} A v_{j} A A v_{k} A\right]
\end{aligned}
$$

Eq. (50) and Eq. (20) differ only in the positions of the two velocity operators and the torque operator between the Green functions. As a consequence, IDOM are ICIDMI are related. In Table I and Table II we list the relations between IDOM and ICIDMI for the Rashba model Eq. (83). We will explain in Sec. III that IDOM and ICIDMI are zero in the Rashba model when the magnetization is along the $z$ direction. Therefore, we discuss in Table@ the case where the magnetization lies in the $x z$ plane, and in Table we discuss the case where the mag-

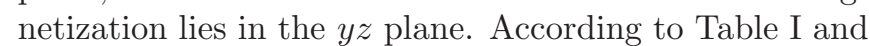
Table III the relation between IDOM and ICIDMI is of the form $\chi_{i j k}^{\mathrm{IDOM}}= \pm \chi_{j i k}^{\mathrm{ICIDMI}}$. This is expected, because the index $i$ in $\chi_{i j k}^{\mathrm{IDOM}}$ is connected to the torque operator, while the index $j$ in $\chi_{i j k}^{\mathrm{ICIDMI}}$ is connected to the torque operator.

TABLE I: Relations between the inverse of the magnetizationdynamics induced orbital magnetism (IDOM) and inverse current-induced DMI (ICIDMI) in the $2 \mathrm{~d}$ Rashba model when $\hat{M}$ lies in the $z x$ plane. The components of $\chi_{i j k}^{\mathrm{IDOM}}$ (Eq. (50)) and $\chi_{i j k}^{\mathrm{ICIDMI}}$ (Eq. 20) are denoted by the three indices $(i j k)$.

\begin{tabular}{c|c}
\hline \hline ICIDMI & IDOM \\
\hline$(211)$ & $(121)$ \\
\hline$(121)$ & $(211)$ \\
\hline$-(221)$ & $(221)$ \\
\hline$(112)$ & $(112)$ \\
\hline$-(212)$ & $(122)$ \\
\hline$-(122)$ & $(212)$ \\
\hline$(222)$ & $(222)$ \\
\hline$(231)$ & $(321)$ \\
\hline$(132)$ & $(312)$ \\
\hline$-(232)$ & $(322)$ \\
\hline
\end{tabular}

TABLE II: Relations between IDOM and ICIDMI in the 2d Rashba model when $\hat{\boldsymbol{M}}$ lies in the $y z$ plane.

\begin{tabular}{c|c}
\hline \hline ICIDMI & IDOM \\
\hline$(111)$ & $(111)$ \\
\hline$-(211)$ & $(121)$ \\
\hline$-(121)$ & $(211)$ \\
\hline$(221)$ & $(221)$ \\
\hline$-(112)$ & $(112)$ \\
\hline$(212)$ & $(122)$ \\
\hline$(122)$ & $(212)$ \\
\hline$-(131)$ & $(311)$ \\
\hline$(231)$ & $(321)$ \\
\hline$(132)$ & $(312)$ \\
\hline \hline
\end{tabular}

\section{F. Contributions from CIDMI and DOM to direct and inverse CIT}

In electronic transport theory the continuity equation determines the current only up to a curl field [37]. The curl of magnetization corresponds to a bound current that cannot be measured in electron transport experiments such that

$$
\boldsymbol{J}=\boldsymbol{J}^{\mathrm{Kubo}}-\nabla \times M
$$

has to be used to extract the transport current $\boldsymbol{J}$ from the current $\boldsymbol{J}^{\text {Kubo }}$ obtained from the Kubo linear response. 
The subtraction of $\boldsymbol{\nabla} \times \boldsymbol{M}$ has been shown to be important when calculating the thermoelectric response 37. and the anomalous Nernst effect [20]. Similarly, in the theory of the thermal spin-orbit torque [10, 18] the gradients of the DMI spiralization have to be subtracted in order to obtain the measurable torque:

$$
T_{i}=T_{i}^{\mathrm{Kubo}}-\sum_{j} \frac{\partial}{\partial r_{j}} D_{i j},
$$

where the spatial derivative of the spiralization arises from its temperature dependence and the temperature gradient.

Since CIDMI and DOM depend on the magnetization direction, they vary spatially in noncollinear magnets. Similar to Eq. (52) the spatial derivatives of the currentinduced spiralization need to be included into the theory of CIT. Additionally, the gradients of DOM correspond to currents that need to be considered in the theory of the inverse CIT, similar to Eq. (51). In section IV we explicitly show that Onsager reciprocity is violated if spatial gradients of DOM and CIDMI are not subtracted from the Kubo response expressions. By trial-and-error we find that the following subtractions are necessary to obtain response currents and torques that satisfy this fundamental symmetry:

$$
J_{i}^{\mathrm{ICIT}}=J_{i}^{\mathrm{Kubo}}-\frac{1}{2} \sum_{j} \frac{\partial \hat{\boldsymbol{M}}}{\partial r_{j}} \cdot \frac{\partial M_{i j}^{\mathrm{DOM}}}{\partial \hat{\boldsymbol{M}}}
$$

and

$$
T_{i}^{\mathrm{CIT}}=T_{i}^{\mathrm{Kubo}}-\frac{1}{2} \sum_{j} \frac{\partial \hat{\boldsymbol{M}}}{\partial r_{j}} \cdot \frac{\partial D_{i j}^{\mathrm{CIDMI}}}{\partial \hat{\boldsymbol{M}}},
$$

where $J_{i}^{\mathrm{ICIT}}$ is the current driven by magnetization dynamics, and $T_{i}^{\mathrm{CIT}}$ is the current-induced torque.

Interestingly, we find that also the diagonal elements $M_{i i}^{\mathrm{DOM}}$ are nonzero. This shows that the generalized definition Eq. (43) is necessary, because the diagonal elements $M_{i i}^{\mathrm{DOM}}$ do not contribute in the usual definition of $M_{i}$ according to Eq. (44). These differences in the symmetry properties between equilibrium and nonequilibrium orbital magnetism can be traced back to symmetry breaking by the perturbations. Also in the case of the spiralization tensor $D_{i j}$ the nonequilibrium correction $\delta D_{i j}$ has different symmetry properties than the equilibrium part (see Sec. III).

The contribution of DOM to $\chi_{i j k l}^{\mathrm{ICIT} 2}$ can be written as

$$
\chi_{i j k l}^{\mathrm{ICIT2c}}=-\frac{1}{2} \hat{\boldsymbol{e}}_{k} \cdot\left[\hat{\boldsymbol{M}} \times \frac{\partial \chi_{j i l}^{\mathrm{DOM}}}{\partial \hat{\boldsymbol{M}}}\right]
$$

and the contribution of CIDMI to $\chi_{i j k l}^{\mathrm{CIT} 2}$ is given by

$$
\chi_{i j k l}^{\mathrm{CIT} 2 \mathrm{~b}}=-\frac{1}{2} \hat{\boldsymbol{e}}_{k} \cdot\left[\hat{\boldsymbol{M}} \times \frac{\partial \chi_{j i l}^{\mathrm{CIDMI}}}{\partial \hat{\boldsymbol{M}}}\right] .
$$

\section{G. Contributions from DDMI to gyromagnetism and damping}

The response to magnetization dynamics that is described by the torque-torque correlation function consists of torques that are related to damping and gyromagnetism [24]. The chiral contribution to these torques can be written as

$$
T_{i}^{\mathrm{TT} 2}=\sum_{j k l} \chi_{i j k l}^{\mathrm{TT} 2} \hat{\boldsymbol{e}}_{j} \cdot\left[\hat{\boldsymbol{M}} \times \frac{\partial \hat{\boldsymbol{M}}}{\partial t}\right] \hat{\boldsymbol{e}}_{k} \cdot\left[\hat{\boldsymbol{M}} \times \frac{\partial \hat{\boldsymbol{M}}}{\partial r_{l}}\right],
$$

where the coefficients $\chi_{i j k l}^{\mathrm{TT} 2}$ satisfy the Onsager relations

$$
\chi_{i j k l}^{\mathrm{TT} 2}(\hat{\boldsymbol{M}})=\chi_{j i k l}^{\mathrm{TT} 2}(-\hat{\boldsymbol{M}}) .
$$

Since DDMI depends on the magnetization direction, it varies spatially in noncollinear magnets and the resulting gradients of DDMI contribute to the damping and to the gyromagnetic ratio:

$$
T_{i}^{\mathrm{TT}}=T_{i}^{\mathrm{Kubo}}-\frac{1}{2} \sum_{j} \frac{\partial \hat{\boldsymbol{M}}}{\partial r_{j}} \cdot \frac{\partial D_{i j}^{\mathrm{DDMI}}}{\partial \hat{\boldsymbol{M}}} .
$$

The resulting contribution of the spatial derivatives of DDMI to the coefficient $\chi_{i j k l}^{\mathrm{TT} 2}$ is

$$
\chi_{i j k l}^{\mathrm{TT} 2 \mathrm{c}}=-\frac{1}{2} \hat{e}_{k} \cdot\left[\hat{\boldsymbol{M}} \times \frac{\partial \chi_{j i l}^{\mathrm{DDMI}}(\hat{\boldsymbol{M}})}{\partial \hat{\boldsymbol{M}}}\right] .
$$

\section{H. Current-induced torque (CIT) in noncollinear magnets}

The chiral contribution to CIT consists of the spatial gradient of CIDMI, $\chi_{i j k l}^{\mathrm{CIT} 2 \mathrm{~b}}$ in Eq. (56), and the Kubo linear response of the torque to the applied electric field in a noncollinear magnet, $\chi_{i j k l}^{\text {CIT2a }}$ :

$$
\chi_{i j k l}^{\mathrm{CIT} 2}=\chi_{i j k l}^{\mathrm{CIT} 2 \mathrm{a}}+\chi_{i j k l}^{\mathrm{CIT} 2 \mathrm{~b}}
$$

In order to determine $\chi_{i j k l}^{\mathrm{CIT} 2 \mathrm{a}}$, we assume that the magnetization direction $\hat{\boldsymbol{M}}(\boldsymbol{r})$ oscillates spatially as described by

$$
\hat{\boldsymbol{M}}(\boldsymbol{r})=\left(\begin{array}{c}
\eta \sin (\boldsymbol{q} \cdot \boldsymbol{r}) \\
0 \\
1
\end{array}\right) \frac{1}{\sqrt{1+\eta^{2} \sin ^{2}(\boldsymbol{q} \cdot \boldsymbol{r})}}
$$

where we will take the limit $\boldsymbol{q} \rightarrow 0$ at the end of the calculation. Since the spatial derivative of the magnetization direction is

$$
\frac{\partial \hat{\boldsymbol{M}}(\boldsymbol{r})}{\partial r_{i}}=\left(\begin{array}{c}
\eta q_{i} \cos (\boldsymbol{q} \cdot \boldsymbol{r}) \\
0 \\
0
\end{array}\right)+\mathcal{O}\left(\eta^{3}\right),
$$


the chiral contribution to the CIT oscillates spatially proportional to $\cos (\boldsymbol{q} \cdot \boldsymbol{r})$. In order to extract this spatially oscillating contribution we multiply with $\cos (\boldsymbol{q} \cdot \boldsymbol{r})$ and integrate over the unit cell. The resulting expression for $\chi_{i j k l}^{\mathrm{CIT2a}}$ is

$$
\begin{aligned}
& \chi_{i j k l}^{\mathrm{CIT2} 2 \mathrm{a}}=-\frac{2 e}{V \eta} \lim _{\boldsymbol{q} \rightarrow 0} \lim _{\omega \rightarrow 0}[ \\
& \left.\frac{1}{q_{l}} \int \cos \left(q_{l} r_{l}\right) \frac{\operatorname{Im}\left\langle\left\langle\mathcal{T}_{i}(\boldsymbol{r}) ; v_{j}\left(\boldsymbol{r}^{\prime}\right)\right\rangle\right\rangle^{\mathrm{R}}(\hbar \omega)}{\hbar \omega} d^{3} r d^{3} r^{\prime}\right],
\end{aligned}
$$

where $V$ is the volume of the unit cell, and the retarded torque-velocity correlation function $\left\langle\left\langle\mathcal{T}_{i}(\boldsymbol{r}) ; v_{j}\left(\boldsymbol{r}^{\prime}\right)\right\rangle\right\rangle^{\mathrm{R}}(\hbar \omega)$ needs to be evaluated in the presence of the perturbation

$$
\delta H=\mathcal{T}_{k} \eta \sin (\boldsymbol{q} \cdot \boldsymbol{r})
$$

due to the noncollinearity (the index $k$ in Eq. 65) needs to match the index $k$ in $\chi_{i j k l}^{\mathrm{CIT} 2 \mathrm{a}}$ ).

In Appendix $\mathrm{B}$ we show that $\chi_{i j k l}^{\mathrm{CIT} 2 \mathrm{a}}$ can be written as

$$
\chi_{i j k l}^{\mathrm{CIT} 2 \mathrm{a}}=-\frac{2 e}{\hbar} \operatorname{Im}\left[\mathscr{W}_{i j k l}^{(\mathrm{surf})}+\mathscr{W}_{i j k l}^{(\mathrm{sea})}\right],
$$

where

$$
\begin{aligned}
\mathscr{W}_{i j k l}^{\text {(surf })} & =\frac{1}{4 \pi \hbar} \int \frac{\mathrm{d}^{d} k}{(2 \pi)^{d}} \int d \mathcal{E} f^{\prime}(\mathcal{E}) \operatorname{Tr}[ \\
& \mathcal{T}_{i} G_{\boldsymbol{k}}^{\mathrm{R}}(\mathcal{E}) v_{l} G_{\boldsymbol{k}}^{\mathrm{R}}(\mathcal{E}) v_{j} G_{\boldsymbol{k}}^{\mathrm{A}}(\mathcal{E}) \mathcal{T}_{k} G_{\boldsymbol{k}}^{\mathrm{A}}(\mathcal{E}) \\
+ & \mathcal{T}_{i} G_{\boldsymbol{k}}^{\mathrm{R}}(\mathcal{E}) v_{j} G_{\boldsymbol{k}}^{\mathrm{A}}(\mathcal{E}) v_{l} G_{\boldsymbol{k}}^{\mathrm{A}}(\mathcal{E}) \mathcal{T}_{k} G_{\boldsymbol{k}}^{\mathrm{A}}(\mathcal{E}) \\
& -\mathcal{T}_{i} G_{\boldsymbol{k}}^{\mathrm{R}}(\mathcal{E}) v_{j} G_{\boldsymbol{k}}^{\mathrm{A}}(\mathcal{E}) \mathcal{T}_{k} G_{\boldsymbol{k}}^{\mathrm{A}}(\mathcal{E}) v_{l} G_{\boldsymbol{k}}^{\mathrm{A}}(\mathcal{E}) \\
+ & \left.\frac{\hbar}{m_{e}} \delta_{j l} \mathcal{T}_{i} G_{\boldsymbol{k}}^{\mathrm{R}}(\mathcal{E}) G_{\boldsymbol{k}}^{\mathrm{A}}(\mathcal{E}) \mathcal{T}_{k} G_{\boldsymbol{k}}^{\mathrm{A}}(\mathcal{E})\right]
\end{aligned}
$$

is a Fermi surface term $\left(f^{\prime}(\mathcal{E})=d f(\mathcal{E}) / d \mathcal{E}\right)$ and

$$
\begin{aligned}
& \mathscr{W}_{i j k l}^{\text {(sea) }}=\frac{1}{4 \pi \hbar^{2}} \int \frac{\mathrm{d}^{d} k}{(2 \pi)^{d}} \int d \mathcal{E} f(\mathcal{E})[ \\
& -\operatorname{Tr}\left[\mathcal{T}_{i} R v_{l} R R v_{j} R \mathcal{T}_{k} R\right]-\operatorname{Tr}\left[\mathcal{T}_{i} R v_{l} R \mathcal{T}_{k} R R v_{j} R\right] \\
& -\operatorname{Tr}\left[\mathcal{T}_{i} R R v_{l} R v_{j} R \mathcal{T}_{k} R\right]-\operatorname{Tr}\left[\mathcal{T}_{i} R R v_{j} R v_{l} R \mathcal{T}_{k} R\right] \\
& +\operatorname{Tr}\left[\mathcal{T}_{i} R R v_{j} R \mathcal{T}_{k} R v_{l} R\right]+\operatorname{Tr}\left[\mathcal{T}_{i} R R \mathcal{T}_{k} R v_{j} R v_{l} R\right] \\
& +\operatorname{Tr}\left[\mathcal{T}_{i} R R \mathcal{T}_{k} R v_{l} R v_{j} R\right]-\operatorname{Tr}\left[\mathcal{T}_{i} R R v_{l} R \mathcal{T}_{k} R v_{j} R\right] \\
& -\operatorname{Tr}\left[\mathcal{T}_{i} R v_{l} R R \mathcal{T}_{k} R v_{j} R\right]+\operatorname{Tr}\left[\mathcal{T}_{i} R \mathcal{T}_{k} R R v_{j} R v_{l} R\right] \\
& +\operatorname{Tr}\left[\mathcal{T}_{i} R \mathcal{T}_{k} R R v_{l} R v_{j} R\right]+\operatorname{Tr}\left[\mathcal{T}_{i} R \mathcal{T}_{k} R v_{l} R R v_{j} R\right] \\
& -\frac{\hbar}{m_{e}} \delta_{j l} \operatorname{Tr}\left[\mathcal{T}_{i} R R R \mathcal{T}_{k} R\right]-\frac{\hbar}{m_{e}} \delta_{j l} \operatorname{Tr}\left[\mathcal{T}_{i} A A A \mathcal{T}_{k} A\right] \\
& \left.-\frac{\hbar}{m_{e}} \delta_{j l} \operatorname{Tr}\left[\mathcal{T}_{i} A A \mathcal{T}_{k} A A\right]\right]
\end{aligned}
$$

is a Fermi sea term.

\section{Inverse CIT in noncollinear magnets}

The chiral contribution $\boldsymbol{J}^{\mathrm{ICIT} 2}$ (see Eq. (4)) to the charge pumping is described by the coefficients

$$
\chi_{i j k l}^{\mathrm{ICITT} 2}=\chi_{i j k l}^{\mathrm{ICIT} 2 \mathrm{a}}+\chi_{i j k l}^{\mathrm{ICIT2} 2 \mathrm{~b}}+\chi_{i j k l}^{\mathrm{ICIT} 2 \mathrm{c}},
$$

where $\chi_{i j k l}^{\mathrm{ICIT} 2 \mathrm{a}}$ describes the response to the timedependent magnetization gradient (see Eq. (18), Eq. (25), and Eq. (24) ) and $\chi_{i j k l}^{\mathrm{ICIT} 2 \mathrm{c}}$ results from the spatial gradient of DOM (see Eq. (55)). $\chi_{i j k l}^{\mathrm{ICIT} 2 \mathrm{~b}}$ describes the response to the perturbation by magnetization dynamics in a noncollinear magnet. In order to derive an expression for $\chi_{i j k l}^{\mathrm{ICIT} 2 \mathrm{~b}}$ we assume that the magnetization oscillates spatially as described by Eq. (62). Since the corresponding response oscillates spatially proportional to $\cos (\boldsymbol{q} \cdot \boldsymbol{r})$, we multiply by $\cos (\boldsymbol{q} \cdot \boldsymbol{r})$ and integrate over the unit cell in order to extract $\chi_{i j k l}^{\mathrm{ICIT} 2 \mathrm{~b}}$ from the retarded velocitytorque correlation function $\left\langle\left\langle v_{i}(\boldsymbol{r}) ; \mathcal{T}_{j}\left(\boldsymbol{r}^{\prime}\right)\right\rangle\right\rangle^{\mathrm{R}}(\hbar \omega)$, which is evaluated in the presence of the perturbation Eq. 65). We obtain

$$
\begin{aligned}
\chi_{i j k l}^{\mathrm{ICIT} 2 \mathrm{~b}} & =\frac{2 e}{V \eta} \lim _{\boldsymbol{q} \rightarrow 0} \lim _{\omega \rightarrow 0}[ \\
& \left.\frac{1}{q_{l}} \int \cos \left(q_{l} r_{l}\right) \frac{\operatorname{Im}\left\langle\left\langle v_{i}(\boldsymbol{r}) ; \mathcal{T}_{j}\left(\boldsymbol{r}^{\prime}\right)\right\rangle\right\rangle^{\mathrm{R}}(\hbar \omega)}{\hbar \omega} d^{3} r d^{3} r^{\prime}\right],
\end{aligned}
$$

which can be written as (see Appendix B)

$$
\chi_{i j k l}^{\mathrm{ICIT2b}}=\frac{2 e}{\hbar} \operatorname{Im}\left[\mathscr{V}_{i j k l}^{(\text {surf })}+\mathscr{V}_{i j k l}^{(\mathrm{sea})}\right],
$$

where

$$
\begin{aligned}
& \mathscr{V}_{i j k l}^{\text {(surf) }}=\frac{1}{4 \pi \hbar} \int \frac{\mathrm{d}^{d} k}{(2 \pi)^{d}} \int d \mathcal{E} f^{\prime}(\mathcal{E}) \operatorname{Tr}[ \\
& \quad v_{i} G_{\boldsymbol{k}}^{\mathrm{R}}(\mathcal{E}) v_{l} G_{\boldsymbol{k}}^{\mathrm{R}}(\mathcal{E}) \mathcal{T}_{j} G_{\boldsymbol{k}}^{\mathrm{A}}(\mathcal{E}) \mathcal{T}_{k} G_{\boldsymbol{k}}^{\mathrm{A}}(\mathcal{E}) \\
& +v_{i} G_{\boldsymbol{k}}^{\mathrm{R}}(\mathcal{E}) \mathcal{T}_{j} G_{\boldsymbol{k}}^{\mathrm{A}}(\mathcal{E}) v_{l} G_{\boldsymbol{k}}^{\mathrm{A}}(\mathcal{E}) \mathcal{T}_{k} G_{\boldsymbol{k}}^{\mathrm{A}}(\mathcal{E}) \\
& \left.-v_{i} G_{\boldsymbol{k}}^{\mathrm{R}}(\mathcal{E}) \mathcal{T}_{j} G_{\boldsymbol{k}}^{\mathrm{A}}(\mathcal{E}) \mathcal{T}_{k} G_{\boldsymbol{k}}^{\mathrm{A}}(\mathcal{E}) v_{l} G_{\boldsymbol{k}}^{\mathrm{A}}(\mathcal{E})\right]
\end{aligned}
$$

is the Fermi surface term and

$$
\begin{aligned}
& \mathscr{V}_{i j k l}^{\text {(sea) }}=\frac{1}{4 \pi \hbar^{2}} \int \frac{\mathrm{d}^{d} k}{(2 \pi)^{d}} \int d \mathcal{E} f(\mathcal{E}) \operatorname{Tr}[ \\
& -\operatorname{Tr}\left[v_{i} R v_{l} R R \mathcal{T}_{j} R \mathcal{T}_{k} R\right]-\operatorname{Tr}\left[v_{i} R v_{l} R \mathcal{T}_{k} R R \mathcal{T}_{j} R\right] \\
& -\operatorname{Tr}\left[v_{i} R R v_{l} R \mathcal{T}_{j} R \mathcal{T}_{k} R\right]-\operatorname{Tr}\left[v_{i} R R \mathcal{T}_{j} R v_{l} R \mathcal{T}_{k} R\right] \\
& +\operatorname{Tr}\left[v_{i} R R \mathcal{T}_{j} R \mathcal{T}_{k} R v_{l} R\right]+\operatorname{Tr}\left[v_{i} R R \mathcal{T}_{k} R \mathcal{T}_{j} R v_{l} R\right] \\
& +\operatorname{Tr}\left[v_{i} R R \mathcal{T}_{k} R v_{l} R \mathcal{T}_{j} R\right]-\operatorname{Tr}\left[v_{i} R R v_{l} R \mathcal{T}_{k} R \mathcal{T}_{j} R\right] \\
& -\operatorname{Tr}\left[v_{i} R v_{l} R R \mathcal{T}_{k} R \mathcal{T}_{j} R\right]+\operatorname{Tr}\left[v_{i} R \mathcal{T}_{k} R R \mathcal{T}_{j} R v_{l} R\right] \\
& \left.+\operatorname{Tr}\left[v_{i} R \mathcal{T}_{k} R R v_{l} R \mathcal{T}_{j} R\right]+\operatorname{Tr}\left[v_{i} R \mathcal{T}_{k} R v_{l} R R \mathcal{T}_{j} R\right]\right]
\end{aligned}
$$

is the Fermi sea term.

In Eq. (70) we use the Kubo formula to describe the response to magnetization dynamics combined with perturbation theory to include the effect of noncollinearity. 
Thereby, the time-dependent perturbation and the perturbation by the magnetization gradient are separated and perturbations of the form of Eq. (15) or Eq. (16) are not automatically included. For example the flat cycloidal spin spiral

$$
\hat{\boldsymbol{M}}(x, t)=\left(\begin{array}{c}
\sin (q x-\omega t) \\
0 \\
\cos (q x-\omega t)
\end{array}\right)
$$

moving in $x$ direction with speed $\omega / q$ and the helical spin spiral

$$
\hat{\boldsymbol{M}}(y, t)=\left(\begin{array}{c}
\sin (q y-\omega t) \\
0 \\
\cos (q y-\omega t)
\end{array}\right)
$$

moving in $y$ direction with speed $\omega / q$ behave like Eq. (10) when $t$ and $\boldsymbol{r}$ are small. Thus, these moving domain walls correspond to the perturbation of the type of Eq. (10) and the resulting contribution $\boldsymbol{J}^{\text {ICIT2a }}$ from the timedependent magnetization gradient is not described by Eq. (70) and needs to be added, which we do by adding $\chi_{i j k l}^{\mathrm{ICIT2a}}$ in Eq. (69).

\section{J. Damping and gyromagnetism in noncollinear magnets}

The chiral contribution Eq. (57) to the torque-torque correlation function is expressed in terms of the coefficient

$$
\chi_{i j k l}^{\mathrm{TT}}=\chi_{i j k l}^{\mathrm{TT} 2 \mathrm{a}}+\chi_{i j k l}^{\mathrm{TT} 2 \mathrm{~b}}+\chi_{i j k l}^{\mathrm{TT} 2 \mathrm{c}},
$$

where $\chi_{i j k l}^{\mathrm{TT} 2 \mathrm{c}}$ results from the spatial gradient of DDMI (see Eq. (60) ), $\chi_{i j k l}^{\mathrm{TT} 2 \mathrm{a}}$ describes the response to a timedependent magnetization gradient in a collinear magnet, and $\chi_{i j k l}^{\mathrm{TT} 2 \mathrm{~b}}$ describes the response to magnetization dynamics in a noncollinear magnet.

In order to derive an expression for $\chi_{i j k l}^{\mathrm{TT} 2 \mathrm{~b}}$ we assume that the magnetization oscillates spatially according to Eq. (62). We multiply the retarded torque-torque correlation function $\left\langle\left\langle\mathcal{T}_{i}(\boldsymbol{r}) ; \mathcal{T}_{j}\left(\boldsymbol{r}^{\prime}\right)\right\rangle\right\rangle^{\mathrm{R}}(\hbar \omega)$ with $\cos \left(q_{l} r_{l}\right)$ and integrate over the unit cell in order to extract the part of the response that varies spatially proportional to $\cos \left(q_{l} r_{l}\right)$. We obtain:

$$
\begin{aligned}
\chi_{i j k l}^{\mathrm{TT} 2 \mathrm{~b}} & =\frac{2}{V \eta} \lim _{q_{l} \rightarrow 0} \lim _{\omega \rightarrow 0}[ \\
& \left.\frac{1}{q_{l}} \int \cos \left(q_{l} r_{l}\right) \frac{\operatorname{Im}\left\langle\left\langle\mathcal{T}_{i}(\boldsymbol{r}) ; \mathcal{T}_{j}\left(\boldsymbol{r}^{\prime}\right)\right\rangle\right\rangle^{\mathrm{R}}(\hbar \omega)}{\hbar \omega} d^{3} r d^{3} r^{\prime}\right] .
\end{aligned}
$$

In Appendix B we discuss how to evaluate Eq. (777) in first order perturbation theory with respect to the perturbation Eq. (65) and show that $\chi_{i j k l}^{\mathrm{TT} 2 \mathrm{~b}}$ can be expressed as

$$
\chi_{i j k l}^{\mathrm{TT} 2 \mathrm{~b}}=\frac{2}{\hbar} \operatorname{Im}\left[\mathscr{X}_{i j k l}^{(\mathrm{surf})}+\mathscr{X}_{i j k l}^{(\mathrm{sea})}\right]
$$

where

$$
\begin{aligned}
\mathscr{X}_{i j k l}^{(\text {surf })} & =\frac{1}{4 \pi \hbar} \int \frac{\mathrm{d}^{d} k}{(2 \pi)^{d}} \int d \mathcal{E} f^{\prime}(\mathcal{E}) \operatorname{Tr}[ \\
& \mathcal{T}_{i} G_{\boldsymbol{k}}^{\mathrm{R}}(\mathcal{E}) v_{l} G_{\boldsymbol{k}}^{\mathrm{R}}(\mathcal{E}) \mathcal{T}_{j} G_{\boldsymbol{k}}^{\mathrm{A}}(\mathcal{E}) \mathcal{T}_{k} G_{\boldsymbol{k}}^{\mathrm{A}}(\mathcal{E}) \\
+ & \mathcal{T}_{i} G_{\boldsymbol{k}}^{\mathrm{R}}(\mathcal{E}) \mathcal{T}_{j} G_{\boldsymbol{k}}^{\mathrm{A}}(\mathcal{E}) v_{l} G_{\boldsymbol{k}}^{\mathrm{A}}(\mathcal{E}) \mathcal{T}_{k} G_{\boldsymbol{k}}^{\mathrm{A}}(\mathcal{E}) \\
- & \left.\mathcal{T}_{i} G_{\boldsymbol{k}}^{\mathrm{R}}(\mathcal{E}) \mathcal{T}_{j} G_{\boldsymbol{k}}^{\mathrm{A}}(\mathcal{E}) \mathcal{T}_{k} G_{\boldsymbol{k}}^{\mathrm{A}}(\mathcal{E}) v_{l} G_{\boldsymbol{k}}^{\mathrm{A}}(\mathcal{E})\right]
\end{aligned}
$$

is a Fermi surface term and

$$
\begin{aligned}
\mathscr{X}_{i j k l}^{\text {(sea) }} & =\frac{1}{4 \pi \hbar^{2}} \int \frac{\mathrm{d}^{d} k}{(2 \pi)^{d}} \int d \mathcal{E} f(\mathcal{E}) \operatorname{Tr}[ \\
& -\left(\mathcal{T}_{i} R v_{l} R R \mathcal{T}_{j} R \mathcal{T}_{k} R\right)-\left(\mathcal{T}_{i} R v_{l} R \mathcal{T}_{k} R R \mathcal{T}_{j} R\right) \\
& -\left(\mathcal{T}_{i} R R v_{l} R \mathcal{T}_{j} R \mathcal{T}_{k} R\right)-\left(\mathcal{T}_{i} R R \mathcal{T}_{j} R v_{l} R \mathcal{T}_{k} R\right) \\
& +\left(\mathcal{T}_{i} R R \mathcal{T}_{j} R \mathcal{T}_{k} R v_{l} R\right)+\left(\mathcal{T}_{i} R R \mathcal{T}_{k} R \mathcal{T}_{j} R v_{l} R\right) \\
& +\left(\mathcal{T}_{i} R R \mathcal{T}_{k} R v_{l} R \mathcal{T}_{j} R\right)-\left(\mathcal{T}_{i} R R v_{l} R \mathcal{T}_{k} R \mathcal{T}_{j} R\right) \\
& -\left(\mathcal{T}_{i} R v_{l} R R \mathcal{T}_{k} R \mathcal{T}_{j} R\right)+\left(\mathcal{T}_{i} R \mathcal{T}_{k} R R \mathcal{T}_{j} R v_{l} R\right) \\
& \left.+\left(\mathcal{T}_{i} R \mathcal{T}_{k} R R v_{l} R \mathcal{T}_{j} R\right)+\left(\mathcal{T}_{i} R \mathcal{T}_{k} R v_{l} R R \mathcal{T}_{j} R\right)\right]
\end{aligned}
$$

is a Fermi sea term.

The contribution $\chi_{i j k l}^{\mathrm{TT} 2 \mathrm{a}}$ from the time-dependent gradients is given by

$$
\chi_{i j k l}^{\mathrm{TT} 2 \mathrm{a}}=-\sum_{m} \chi_{i m l}^{\mathrm{TT} 2 \mathrm{a}}\left[1-\delta_{j m}\right] \delta_{j k},
$$

where

$$
\begin{gathered}
\chi_{i m l}^{\mathrm{TT} 2 \mathrm{a}}=\frac{i}{4 \pi \hbar^{2}} \int \frac{\mathrm{d}^{d} k}{(2 \pi)^{d}} \int d \mathcal{E} f(\mathcal{E}) \operatorname{Tr}[ \\
\mathcal{T}_{i} R v_{l} R R \mathcal{O}_{m} R+\mathcal{T}_{i} R R v_{l} R \mathcal{O}_{m} R+ \\
-\mathcal{T}_{i} R R \mathcal{O}_{m} R v_{l} R-\mathcal{T}_{i} R v_{l} R \mathcal{O}_{m} A A \\
+\mathcal{T}_{i} R \mathcal{O}_{m} A v_{l} A A+\mathcal{T}_{i} R \mathcal{O}_{m} A A v_{l} A \\
-\mathcal{T}_{i} R v_{l} R R \mathcal{O}_{m} A-\mathcal{T}_{i} R R v_{l} R \mathcal{O}_{m} A \\
+\mathcal{T}_{i} R R \mathcal{O}_{m} A v_{l} A+\mathcal{T}_{i} A v_{l} A \mathcal{O}_{m} A A \\
\left.-\mathcal{T}_{i} A \mathcal{O}_{m} A v_{l} A A-\mathcal{T}_{i} A \mathcal{O}_{m} A A v_{l} A\right]
\end{gathered}
$$

with $\mathcal{O}_{m}=\partial H / \partial \hat{M}_{m}$ (see Appendix $\mathrm{A}$ ).

\section{SYMMETRY PROPERTIES}

In this section we discuss the symmetry properties of CIDMI, DDMI and DOM in the case of the magnetic Rashba model

$$
H_{\boldsymbol{k}}(\boldsymbol{r})=\frac{\hbar^{2}}{2 m_{e}} k^{2}+\alpha\left(\boldsymbol{k} \times \hat{\boldsymbol{e}}_{z}\right) \cdot \boldsymbol{\sigma}+\frac{\Delta V}{2} \boldsymbol{\sigma} \cdot \hat{\boldsymbol{M}}(\boldsymbol{r}) .
$$


Additionally, we discuss the symmetry properties of the currents and torques induced by time-dependent magnetization gradients of the form of Eq. (10).

We consider mirror reflection $\mathcal{M}_{x z}$ at the $x z$ plane, mirror reflection $\mathcal{M}_{y z}$ at the $y z$ plane, and c2 rotation around the $z$ axis. When $\Delta V=0$ these operations leave Eq. (83) invariant, but when $\Delta V \neq 0$ they modify the magnetization direction $\hat{M}$ in Eq. (83), as shown in Table III At the same time, these operations affect the torque $\boldsymbol{T}$ and the current $\boldsymbol{J}$ driven by the time-dependent magnetization gradients (see Table 【II). In Table【V and Table $\mathbf{V}$ we show how $\hat{\boldsymbol{M}} \times \partial \hat{\boldsymbol{M}} / \partial r_{k}$ is affected by the symmetry operations.

A flat cycloidal spin spiral with spins rotating in the $x z$ plane is mapped by a c2 rotation around the $z$ axis onto the same spin spiral. Similarly, a flat helical spin spiral with spins rotating in the $y z$ plane is mapped by a c2 rotation around the $z$ axis onto the same spin spiral. Therefore, when $\hat{M}$ points in $z$ direction, a c2 rotation around the $z$ axis does not change $\hat{\boldsymbol{M}} \times \partial \hat{\boldsymbol{M}} / \partial r_{i}$, but it flips the in-plane current $\boldsymbol{J}$ and the in-plane components of the torque, $T_{x}$ and $T_{y}$. Consequently, $\hat{\boldsymbol{M}} \times \partial^{2} \hat{\boldsymbol{M}} / \partial r_{i} \partial t$ does not induce currents or torques, i.e., ICIDMI, CIDMI, IDDMI and DDMI are zero, when $\hat{\boldsymbol{M}}$ points in $z$ direction. However, they become nonzero when the magnetization has an in-plane component (see Fig. 1).

Similarly, IDOM vanishes when the magnetization points in $z$ direction: In that case Eq. (83) is invariant under the $\mathrm{c} 2$ rotation. A time-dependent magnetic field along $z$ direction is invariant under the $\mathrm{c} 2$ rotation as well. However, $T_{x}$ and $T_{y}$ change sign under the $\mathrm{c} 2$ rotation. Consequently, symmetry forbids IDOM in this case. However, when the magnetization has an in-plane component, IDOM and DOM become nonzero (see Fig. 2).

That time-dependent magnetization gradients of the type of Eq. (7) do not induce in-plane currents and torques when $\hat{\boldsymbol{M}}$ points in $z$ direction can also be seen directly from Eq. (7): The c2 rotation transforms $\boldsymbol{q} \rightarrow-\boldsymbol{q}$ and $M_{x} \rightarrow-M_{x}$. Since $\sin (\boldsymbol{q} \cdot \boldsymbol{r})$ is odd in $\boldsymbol{r}$, Eq. (77) is invariant under $\mathrm{c} 2$ rotation, while the in-plane currents and torques induced by time-dependent magnetization gradients change sign under $\mathrm{c} 2$ rotation. In contrast, Eq. (10) is not invariant under $\mathrm{c} 2$ rotation, because $\sin (\boldsymbol{q} \cdot \boldsymbol{r}-\omega t)$ is not odd in $\boldsymbol{r}$ for $t>0$. Consequently, time-dependent magnetization gradients of the type of Eq. (10) induce currents and torques also when $\hat{M}$ points locally into the $z$ direction. These currents and torques, which are described by Eq. (24) and Eq. (82), respectively, need to be added to the chiral ICIT and the chiral torque-torque correlation. While CIDMI, DDMI, and DOM are zero when the magnetization points in $z$ direction, their gradients are not (see Fig. 11 and Fig. 22). Therefore, the gradients of CIDMI, DOM, and DDMI contribute to CIT, to ICIT and to the torque-torque correlation, respectively, even when $\hat{M}$ points locally into the $z$ direction.
TABLE III: Effect of mirror reflection $\mathcal{M}_{x z}$ at the $x z$ plane, mirror reflection $\mathcal{M}_{y z}$ at the $y z$ plane, and c2 rotation around the $z$ axis. The magnetization $\boldsymbol{M}$ and the torque $\boldsymbol{T}$ transform like axial vectors, while the current $\boldsymbol{J}$ transforms like a polar vector.

\begin{tabular}{c|c|c|c|c|c|c|c|c}
\hline \hline & $M_{x}$ & $M_{y}$ & $M_{z}$ & $J_{x}$ & $J_{y}$ & $T_{x}$ & $T_{y}$ & $T_{z}$ \\
\hline $\mathcal{M}_{x z}$ & $-M_{x}$ & $M_{y}$ & $-M_{z}$ & $J_{x}$ & $-J_{y}$ & $-T_{x}$ & $T_{y}$ & $-T_{z}$ \\
\hline $\mathcal{M}_{y z}$ & $M_{x}$ & $-M_{y}$ & $-M_{z}$ & $-J_{x}$ & $J_{y}$ & $T_{x}$ & $-T_{y}$ & $-T_{z}$ \\
\hline $\mathrm{c} 2$ & $-M_{x}$ & $-M_{y}$ & $M_{z}$ & $-J_{x}$ & $-J_{y}$ & $-T_{x}$ & $-T_{y}$ & $T_{z}$ \\
\hline \hline
\end{tabular}

TABLE IV: Effect of symmetry operations on the magnetization gradients. Magnetization gradients are described by three indices $(i j k)$. The first index denotes the magnetization direction at $\boldsymbol{r}=0$. The third index denotes the direction along which the magnetization changes. The second index denotes the direction of $\partial \hat{M} / \partial r_{k} \delta r_{k}$. The direction of $\hat{\boldsymbol{M}} \times \partial \hat{\boldsymbol{M}} / \partial r_{k}$ is specified by the number below the indices $(i j k)$.

\begin{tabular}{c|c|c|c|c|c|c}
\hline \hline & $(1,2,1)$ & $(1,3,1)$ & $(2,1,1)$ & $(2,3,1)$ & $(3,1,1)$ & $(3,2,1)$ \\
& 3 & -2 & -3 & 1 & 2 & -1 \\
\hline $\mathcal{M}_{x z}$ & $(-1,2,1)$ & $(-1,-3,1)$ & $(2,-1,1)$ & $(2,-3,1)$ & $(-3,-1,1)$ & $(-3,2,1)$ \\
& -3 & -2 & 3 & -1 & 2 & 1 \\
\hline $\mathcal{M}_{y z}$ & $(1,2,1)$ & $(1,3,1)$ & $(-2,-1,1)$ & $(-2,3,1)$ & $(-3,-1,1)$ & $(-3,2,1)$ \\
& 3 & -2 & -3 & -1 & 2 & 1 \\
\hline $\mathrm{c} 2$ & $(-1,2,1)$ & $(-1,-3,1)$ & $(-2,1,1)$ & $(-2,-3,1)$ & $(3,1,1)$ & $(3,2,1)$ \\
& -3 & -2 & 3 & 1 & 2 & -1 \\
\hline \hline
\end{tabular}

TABLE V: Continuation of Table IV

\begin{tabular}{c|c|c|c|c|c|c}
\hline \hline & $(1,2,2)$ & $(1,3,2)$ & $(2,1,2)$ & $(2,3,2)$ & $(3,1,2)$ & $(3,2,2)$ \\
& 3 & -2 & -3 & 1 & 2 & -1 \\
\hline $\mathcal{M}_{x z}$ & $(-1,-2,2)$ & $(-1,3,2)$ & $(2,1,2)$ & $(2,3,2)$ & $(-3,1,2)$ & $(-3,-2,2)$ \\
& 3 & 2 & -3 & 1 & -2 & -1 \\
\hline $\mathcal{M}_{y z}$ & $(1,-2,2)$ & $(1,-3,2)$ & $(-2,1,2)$ & $(-2,-3,2)$ & $(-3,1,2)$ & $(-3,-2,2)$ \\
& -3 & 2 & 3 & 1 & -2 & -1 \\
\hline $\mathrm{c} 2$ & $(-1,2,2)$ & $(-1,-3,2)$ & $(-2,1,2)$ & $(-2,-3,2)$ & $(3,1,2)$ & $(3,2,2)$ \\
& -3 & -2 & 3 & 1 & 2 & -1 \\
\hline \hline
\end{tabular}

\section{A. Symmetry properties of ICIDMI and IDDMI}

In the following we discuss how Table [II, Table[V] and Table $\square$ can be used to analyze the symmetry of ICIDMI and IDDMI. According to Eq. (19) the coefficient $\chi_{i j k}^{\text {ICIDMI }}$ describes the response of the current $J_{i}^{\mathrm{ICIT} 2 \mathrm{a}}$ to the timedependent magnetization gradient $\hat{\boldsymbol{e}}_{j} \cdot\left[\hat{\boldsymbol{M}} \times \frac{\partial^{2} \hat{\boldsymbol{M}}}{\partial r_{k} \partial t}\right]$. Since $\hat{\boldsymbol{M}} \times \frac{\partial^{2} \hat{M}}{\partial r_{k} \partial t}=\frac{\partial}{\partial t}\left[\hat{\boldsymbol{M}} \times \frac{\partial \hat{M}}{\partial r_{k}}\right]$ for time-dependent magnetization gradients of the type Eq. (7) the symmetry properties of $\chi_{i j k}^{\text {ICIDMI }}$ follow from the transformation behaviour of $\hat{\boldsymbol{M}} \times \frac{\partial \hat{M}}{\partial r_{k}}$ and $\boldsymbol{J}$ under symmetry operations.

We consider the case with magnetization in $x$ direction. The component $\chi_{132}^{\text {ICIDMI }}$ describes the current in $x$ direction induced by the time-dependence of a cycloidal magnetization gradient in $y$ direction (with spins rotating 
(a)
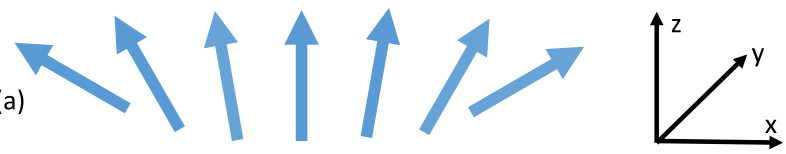

(b)

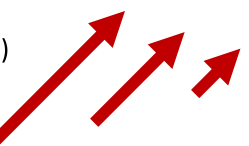

FIG. 1: ICIDMI in a noncollinear magnet. (a) Arrows illustrate the magnetization direction. (b) Arrows illustrate the current $J_{y}$ induced by a time-dependent magnetization gradient, which is described by $\chi_{221}^{\text {ICIDMI }}$. When $\hat{\boldsymbol{M}}$ points in $z$ direction, $\chi_{221}^{\text {ICIDMI }}$ and $J_{y}$ are zero. The sign of $\chi_{221}^{\text {ICIDMI }}$ and of $J_{y}$ changes with the sign of $M_{x}$.

(a)
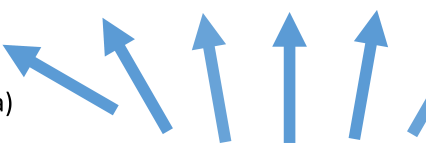

(b)

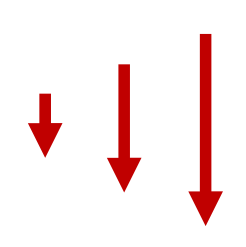

FIG. 2: DOM in a noncollinear magnet. (a) Arrows illustrate the magnetization direction. (b) Arrows illustrate the orbital magnetization induced by magnetization dynamics (DOM). When $\hat{M}$ points in $z$ direction, DOM is zero. The sign of DOM changes with the sign of $M_{x}$.

in the $x y$ plane). $\mathcal{M}_{y z}$ flips both $\hat{\boldsymbol{M}} \times \frac{\partial \hat{M}}{\partial y}$ and $J_{x}$, but it preserves $\hat{\boldsymbol{M}} . \mathcal{M}_{z x}$ preserves $\hat{\boldsymbol{M}} \times \frac{\partial \hat{M}}{\partial y}$ and $J_{x}$, but it flips $\hat{\boldsymbol{M}}$. A c2 rotation around the $z$ axis flips $\hat{\boldsymbol{M}} \times \frac{\partial \hat{M}}{\partial y}$, $\hat{\boldsymbol{M}}$ and $J_{x}$. Consequently, $\chi_{132}^{\mathrm{ICIDMI}}(\hat{\boldsymbol{M}})$ is allowed by symmetry and it is even in $\hat{\boldsymbol{M}}$. The component $\chi_{122}^{\mathrm{ICIDMI}}$ describes the current in $x$ direction induced by the timedependence of a helical magnetization gradient in $y$ direction (with spins rotating in the $x z$ plane). $\mathcal{M}_{y z}$ flips $\hat{\boldsymbol{M}} \times \frac{\partial \hat{M}}{\partial y}$ and $J_{x}$, but it preserves $\hat{\boldsymbol{M}} . \mathcal{M}_{z x}$ flips $\hat{\boldsymbol{M}} \times \frac{\partial \hat{M}}{\partial y}$ and $\hat{\boldsymbol{M}}$, but it preserves $J_{x}$. A c2 rotation around the $z$ axis flips $J_{x}$ and $\hat{\boldsymbol{M}}$, but it preserves $\hat{\boldsymbol{M}} \times \frac{\partial \hat{M}}{\partial y}$. Consequently, $\chi_{122}^{\mathrm{ICIDMI}}$ is allowed by symmetry and it is odd in $\hat{\boldsymbol{M}}$. The component $\chi_{221}^{\mathrm{ICIDMI}}$ describes the current in $y$ direction induced by the time-dependence of a cycloidal magnetization gradient in $x$ direction (with spins rotating in the $x z$ plane). $\mathcal{M}_{z x}$ preserves $\hat{M} \times \frac{\partial \hat{M}}{\partial x}$, but it flips
$J_{y}$ and $\hat{\boldsymbol{M}} . \mathcal{M}_{y z}$ preserves $\hat{\boldsymbol{M}}, J_{y}$, and $\hat{\boldsymbol{M}} \times \frac{\partial \hat{M}}{\partial x}$. The c2 rotation around the $z$ axis preserves $\hat{M} \times \frac{\partial \hat{M}}{\partial x}$, but it flips $\hat{\boldsymbol{M}}$ and $J_{y}$. Consequently, $\chi_{221}^{\mathrm{ICIDMI}}$ is allowed by symmetry and it is odd in $\hat{\boldsymbol{M}}$. The component $\chi_{231}^{\mathrm{ICIDMI}}$ describes the current in $y$ direction induced by the timedependence of a cycloidal magnetization gradient in $x$ direction (with spins rotating in the $x y$ plane). $\mathcal{M}_{z x}$ flips $\hat{\boldsymbol{M}} \times \frac{\partial \hat{M}}{\partial x}, \hat{M}$, and $J_{y} . \mathcal{M}_{y z}$ preserves $\hat{\boldsymbol{M}} \times \frac{\partial \hat{M}}{\partial x}, \hat{M}$ and $J_{y}$. The $\mathrm{c} 2$ rotation around the $z$ axis flips $\hat{\boldsymbol{M}} \times \frac{\partial \hat{M}}{\partial x}, J_{y}$, and $\hat{M}$. Consequently, $\chi_{231}^{\text {ICIDMI }}$ is allowed by symmetry and it is even in $\hat{\boldsymbol{M}}$.

These properties are summarized in Table VI, Due to the relations between CIDMI and DOM (see Table $\square$ and Table II), they can be used for DOM as well. When the magnetization lies at a general angle in the $x z$ plane or in the $y z$ plane several additional components of CIDMI and DOM are nonzero (see Table凹and Table【 respectively).

TABLE VI: Allowed components of $\chi_{i j k}^{\mathrm{ICIDMI}}$ when $\hat{\boldsymbol{M}}$ points in $x$ direction. + components are even in $\hat{\boldsymbol{M}}$, while - components are odd in $\hat{\boldsymbol{M}}$.

\begin{tabular}{c|c|c|c}
\hline \hline 132 & 122 & 221 & 231 \\
\hline+ & - & - & + \\
\hline \hline
\end{tabular}

Similarly, one can analyze the symmetry of DDMI. Table VII lists the components of DDMI, $\chi_{i j k}^{\mathrm{DDMI}}$, which are allowed by symmetry when $\hat{M}$ points in $x$ direction.

TABLE VII: Allowed components of $\chi_{i j k}^{\text {DDMI }}$ when $\hat{M}$ points in $x$ direction. + components are even in $\hat{M}$, while - components are odd in $\hat{\boldsymbol{M}}$.

\begin{tabular}{c|c|c|c}
\hline \hline 222 & 232 & 322 & 332 \\
\hline- & + & + & - \\
\hline \hline
\end{tabular}

B. Response to time-dependent magnetization gradients of the second type (Eq. (10)

According to Eq. (13) the time-dependent magnetization gradient is along the magnetization. Therefore, in contrast to the discussion in section IIIA we cannot use $\hat{\boldsymbol{M}} \times \frac{\partial^{2} \hat{M}}{\partial r_{k} \partial t}$ in the symmetry analysis. Eq. (24) and Eq. (25) show that $\chi_{i j j l}^{\mathrm{ICIT} 2 \mathrm{a}}$ describes the response of $J_{i}^{\mathrm{ICIT} 2 \mathrm{a}}$ to $\hat{\boldsymbol{e}}_{j} \cdot\left[\hat{\boldsymbol{M}} \times \frac{\partial \hat{\boldsymbol{M}}}{\partial t}\right] \hat{\boldsymbol{e}}_{j} \cdot\left[\hat{\boldsymbol{M}} \times \frac{\partial \hat{\boldsymbol{M}}}{\partial r_{l}}\right]$ while $\chi_{i j k l}^{\mathrm{ICIT2a}}=$ 0 for $j \neq k$. According to Eq. (23) the symmetry properties of $\left[\hat{M} \times \frac{\partial \hat{M}}{\partial t}\right] \cdot\left[\hat{M} \times \frac{\partial \hat{M}}{\partial r_{l}}\right]$ agree to the symmetry properties of $\hat{\boldsymbol{M}} \cdot \frac{\partial^{2} \hat{M}}{\partial r_{l} \partial t}$. Therefore, in order to understand the symmetry properties of $\chi_{i j j l}^{\mathrm{ICIT2a}}$ we consider the transformation of $\boldsymbol{J}$ and $\hat{\boldsymbol{M}} \cdot \frac{\partial^{2} \hat{\boldsymbol{M}}}{\partial r_{l} \partial t}$ under symmetry operations.

We consider the case where $\hat{M}$ points in $z$ direction. $\chi_{1 j j 1}^{\mathrm{ICIT2a}}$ describes the current driven in $x$ direction, when 
the magnetization varies in $x$ direction. $\mathcal{M}_{x z}$ flips $\hat{\boldsymbol{M}}$, but preserves $J_{x}$ and $\hat{\boldsymbol{M}} \cdot \partial^{2} \hat{\boldsymbol{M}} /(\partial x \partial t)$. $\mathcal{M}_{y z}$ flips $\hat{\boldsymbol{M}}, J_{x}$, and $\hat{\boldsymbol{M}} \cdot \partial^{2} \hat{\boldsymbol{M}} /(\partial x \partial t)$. c2 rotation flips $\hat{\boldsymbol{M}} \cdot \partial^{2} \hat{\boldsymbol{M}} /(\partial x \partial t)$ and $J_{x}$, but preserves $\hat{M}$. Consequently, $\chi_{1 j j 1}^{\text {ICIT2a }}$ is allowed by symmetry and it is even in $\hat{M}$.

$\chi_{2 j j 1}^{\mathrm{ICIT} 2 \mathrm{a}}$ describes the current flowing in $y$ direction, when magnetization varies in $x$ direction. $\mathcal{M}_{x z}$ flips $\hat{\boldsymbol{M}}$ and $J_{y}$, but preserves $\hat{\boldsymbol{M}} \cdot \partial^{2} \hat{\boldsymbol{M}} /(\partial x \partial t) . \mathcal{M}_{y z}$ flips $\hat{\boldsymbol{M}}$, and $\hat{\boldsymbol{M}} \cdot \partial^{2} \hat{\boldsymbol{M}} /(\partial x \partial t)$, but preserves $J_{y}$. c2 rotation flips $\hat{\boldsymbol{M}} \cdot \partial^{2} \hat{\boldsymbol{M}} /(\partial x \partial t)$ and $J_{y}$, but preserves $\hat{\boldsymbol{M}}$. Consequently, $\chi_{2 j j 1}^{\mathrm{ICIT} 2 \mathrm{a}}$ is allowed by symmetry and it is odd in $\hat{M}$.

Similarly, one can show that $\chi_{1 j j 2}^{\mathrm{ICIT} 2 \mathrm{a}}$ is odd in $\hat{\boldsymbol{M}}$ and that $\chi_{2 j j 2}^{\mathrm{ICIT} 2 \mathrm{a}}$ is even in $\hat{\boldsymbol{M}}$.

Analogously, one can investigate the symmetry properties of $\chi_{i j j l}^{\mathrm{TT} 2 \mathrm{a}}$. We find that $\chi_{1 j j 1}^{\mathrm{TT} 2 \mathrm{a}}$ and $\chi_{2 j j 2}^{\mathrm{TT} 2 \mathrm{a}}$ are odd in $\hat{\boldsymbol{M}}$, while $\chi_{2 j j 1}^{\mathrm{TT} 2 \mathrm{a}}$ and $\chi_{1 j j 2}^{\mathrm{TT} 2 \mathrm{a}}$ are even in $\hat{\boldsymbol{M}}$.

\section{RESULTS}

In the following sections we discuss the results for the direct and inverse chiral CIT and for the chiral torquetorque correlation in the two-dimensional (2d) Rashba model Eq. (83), and in the one-dimensional (1d) Rashba model [38]

$$
H_{k_{x}}(x)=\frac{\hbar^{2}}{2 m_{e}} k_{x}^{2}-\alpha k_{x} \sigma_{y}+\frac{\Delta V}{2} \boldsymbol{\sigma} \cdot \hat{\boldsymbol{M}}(x) .
$$

Additionally, we discuss the contributions of the timedependent magnetization gradients, and of DDMI, DOM and CIDMI to these effects.

While vertex corrections to the chiral CIT and to the chiral torque-torque correlation are important in the Rashba model [38], the purpose of this work is to show the importance of the contributions from time-dependent magnetization gradients, DDMI, DOM and CIDMI. We therefore consider only the intrinsic contributions here, i.e., we set

$$
G_{\boldsymbol{k}}^{\mathrm{R}}(\mathcal{E})=\hbar\left[\mathcal{E}-H_{\boldsymbol{k}}+i \Gamma\right]^{-1},
$$

where $\Gamma$ is a constant broadening, and we leave the study of vertex corrections for future work.

The results shown in the following sections are obtained for the model parameters $\Delta V=1 \mathrm{eV}, \alpha=2 \mathrm{eV} \AA$, and $\Gamma=0.1 \mathrm{Ry}=1.361 \mathrm{eV}$, when the magnetization points in $z$ direction, i.e., $\hat{\boldsymbol{M}}=\hat{\boldsymbol{e}}_{z}$. The unit of $\chi_{i j k l}^{\mathrm{CIT} 2}$ is charge times length in the $1 \mathrm{~d}$ case and charge in the $2 \mathrm{~d}$ case. Therefore, in the $1 \mathrm{~d}$ case we discuss the chiral torkance in units of $e a_{0}$, where $a_{0}$ is Bohr's radius. In the $2 \mathrm{~d}$ case we discuss the chiral torkance in units of $e$. The unit of $\chi_{i j k l}^{\mathrm{TT} 2}$ is angular momentum in the $1 \mathrm{~d}$ case and angular momentum per length in the $2 \mathrm{~d}$ case. Therefore, we discuss $\chi_{i j k l}^{\mathrm{TT} 2}$ in units of $\hbar$ in the $1 \mathrm{~d}$ case, and in units of $\hbar / a_{0}$ in the $2 \mathrm{~d}$ case.

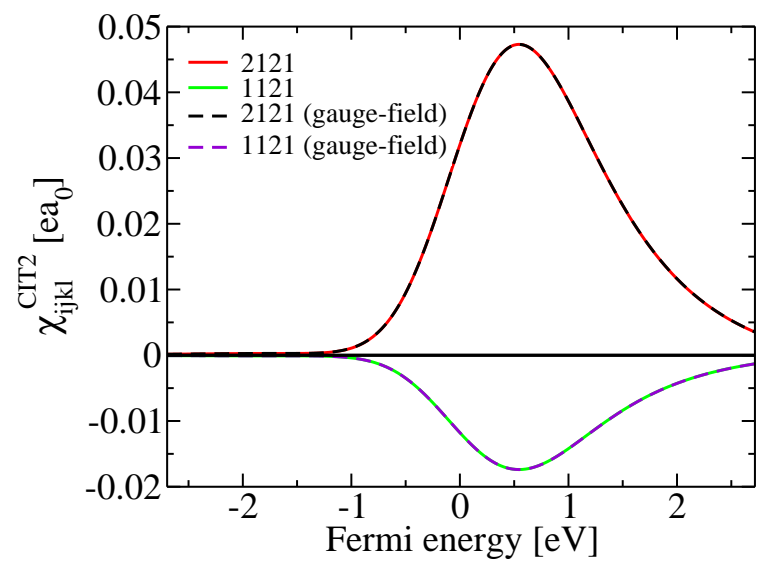

FIG. 3: Chiral CIT in the 1d Rashba model for cycloidal gradients vs. Fermi energy. General perturbation theory (solid lines) agrees to the gauge-field approach (dashed lines).

\section{A. Direct and inverse chiral CIT}

In Fig. 3 we show the chiral CIT as a function of the Fermi energy for cycloidal magnetization gradients in the 1d Rashba model. The components $\chi_{2121}^{\mathrm{CIT} 2}$ and $\chi_{1121}^{\mathrm{CIT2}}$ are labelled by 2121 and 1121, respectively. The component 2121 of CIT describes the non-adiabatic torque, while the component 1121 describes the adiabatic STT (modified by SOI). In the one-dimensional Rashba model, the contributions $\chi_{2121}^{\mathrm{CIT} 2 \mathrm{~b}}$ and $\chi_{1121}^{\mathrm{CIT} 2 \mathrm{~b}}$ (Eq. (56) ) from the CIDMI are zero when $\hat{\boldsymbol{M}}=\hat{\boldsymbol{e}}_{z}$ (not shown in the figure). For cycloidal spin spirals, it is possible to solve the 1d Rashba model by a gauge-field approach [38], which allows us to test the perturbation theory, Eq. (66). For comparison we show in Fig. 3 the results obtained from the gaugefield approach, which agree to the perturbation theory, Eq. (66). This demonstrates the validity of Eq. (66).

In Fig. 4 we show the chiral ICIT in the 1d Rashba model. The components $\chi_{1221}^{\mathrm{ICIT} 2}$ and $\chi_{1121}^{\mathrm{ICIT} 2}$ are labelled by 1221 and 1121, respectively. The contribution $\chi_{1221}^{\mathrm{ICIT2a}}$ from the time-dependent gradient is of the same order of magnitude as the total $\chi_{1221}^{\mathrm{ICIT} 2}$. Comparison of Fig. 3 and Fig. 4 shows that CIT and ICIT satisfy the reciprocity relations Eq. (5), that $\chi_{1121}^{\mathrm{CIT} 2}$ is odd in $\hat{\boldsymbol{M}}$, and that $\chi_{2121}^{\mathrm{CIT} 2}$ is even in $\hat{\boldsymbol{M}}$, i.e., $\chi_{2121}^{\mathrm{CIT} 2}=\chi_{1221}^{\mathrm{ICIT} 2}$ and $\chi_{1121}^{\mathrm{CIT} 2}=-\chi_{1121}^{\mathrm{ICIT} 2}$. The contribution $\chi_{1221}^{\mathrm{ICI} I \mathrm{La}}$ from the time-dependent gradients is crucial to satisfy the reciprocity relations between $\chi_{2121}^{\mathrm{CIT} 2}$ and $\chi_{1221}^{\mathrm{ICIT} 2}$.

In Fig. 5] and Fig. 6 we show the CIT and the ICIT, respectively, for helical gradients in the 1d Rashba model. The components $\chi_{2111}^{\mathrm{CIT} 2}$ and $\chi_{1111}^{\mathrm{CIT} 2}$ are labelled 2111 and 1111, respectively, in Fig. 5, while $\chi_{1211}^{\mathrm{ICIT} 2}$ and $\chi_{1111}^{\mathrm{ICIT} 2}$ are labelled 1211 and 1111, respectively, in Fig. 6. The contributions $\chi_{2111}^{\mathrm{CIT} 2 \mathrm{~b}}$ and $\chi_{1111}^{\mathrm{CIT} 2 \mathrm{~b}}$ from CIDMI are of the 


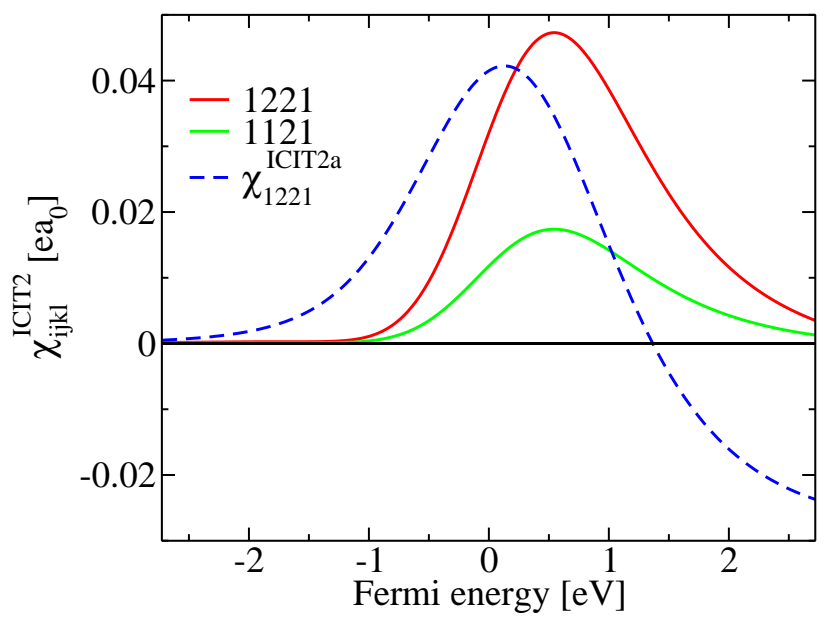

FIG. 4: Chiral ICIT in the 1d Rashba model for cycloidal gradients vs. Fermi energy. Dashed line: Contribution from the time-dependent gradient.

same order of magnitude as the total $\chi_{2111}^{\mathrm{CIT} 2}$ and $\chi_{1111}^{\mathrm{CIT} 2}$. Similarly, the contributions $\chi_{1211}^{\mathrm{ICIT} 2 \mathrm{c}}$ and $\chi_{1111}^{\mathrm{ICIT} 2 \mathrm{c}}$ from DOM are of the same order of magnitude as the total $\chi_{1211}^{\mathrm{ICIT} 2}$ and $\chi_{1111}^{\mathrm{ICIT} 2}$. Additionally, the contribution $\chi_{1111}^{\mathrm{ICIT} 2 \mathrm{a}}$ from the time-dependent gradient is substantial. Comparison of Fig. [5 and Fig. [6 shows that CIT and ICIT satisfy the reciprocity relation Eq. (5), that $\chi_{2111}^{\mathrm{CIT} 2}$ is odd in $\hat{\boldsymbol{M}}$, and that $\chi_{1111}^{\mathrm{CIT} 2}$ is even in $\hat{\boldsymbol{M}}$, i.e., $\chi_{1111}^{\mathrm{CIT} 2}=\chi_{1111}^{\mathrm{ICIT} 2}$ and $\chi_{2111}^{\mathrm{CIT} 2}=-\chi_{1211}^{\mathrm{ICIT} 2}$. These reciprocity relations between CIT and ICIT are only satisfied when CIDMI, DOM, and the response to time-dependent magnetization gradients are included. Additionally, the comparison between Fig. 5 and Fig. 6 shows that the contributions of CIDMI to CIT $\left(\chi_{1111}^{\mathrm{CIT} 2 \mathrm{~b}}\right.$ and $\left.\chi_{2111}^{\mathrm{CIT} 2 \mathrm{~b}}\right)$ are related to the contributions of DOM to ICIT $\left(\chi_{1111}^{\mathrm{ICIT} 2 \mathrm{c}}\right.$ and $\left.\chi_{1211}^{\text {ICIT2c }}\right)$. These relations between DOM and ICIT are expected from Table

In Fig. 7 and Fig. 8 we show the CIT and the ICIT, respectively, for cycloidal gradients in the $2 \mathrm{~d}$ Rashba model. In this case there are contributions from CIDMI and DOM in contrast to the $1 \mathrm{~d}$ case with cycloidal gradients (Fig. 3). Comparison between Fig. 7 and Fig. 8 shows that $\chi_{1121}^{\mathrm{CIT} 2}$ and $\chi_{2221}^{\mathrm{CIT} 2}$ are odd in $\hat{\boldsymbol{M}}$, that $\chi_{1221}^{\mathrm{CIT} 2}$ and $\chi_{2121}^{\mathrm{CIT} 2}$ are even in $\boldsymbol{M}$, and that CIT and ICIT satisfy the reciprocity relation Eq. (5) when the gradients of CIDMI and DOM are included, i.e., $\chi_{1121}^{\mathrm{CIT} 2}=-\chi_{1121}^{\mathrm{ICIT} 2}$, $\chi_{2221}^{\mathrm{CIT} 2}=-\chi_{2221}^{\mathrm{ICIT2}}, \chi_{1221}^{\mathrm{CIT2}}=\chi_{2121}^{\mathrm{ICIT} 2}$, and $\chi_{2121}^{\mathrm{CIT} 2}=\chi_{1221}^{\mathrm{ICIT} 2}$. $\chi_{1121}^{\mathrm{CIT2}}$ describes the adiabatic STT with SOI, while $\chi_{2121}^{\mathrm{CIT} 2}$ describes the non-adiabatic STT. Experimentally, it has been found that CITs occur also when the electric field is applied parallel to domain-walls (i.e., perpendicular to the $\boldsymbol{q}$-vector of spin spirals) 39]. In our calculations, the components $\chi_{2221}^{\mathrm{CIT} 2}$ and $\chi_{1221}^{\mathrm{CIT} 2}$ describe such a case, where the applied electric field points in $y$ direction, while the

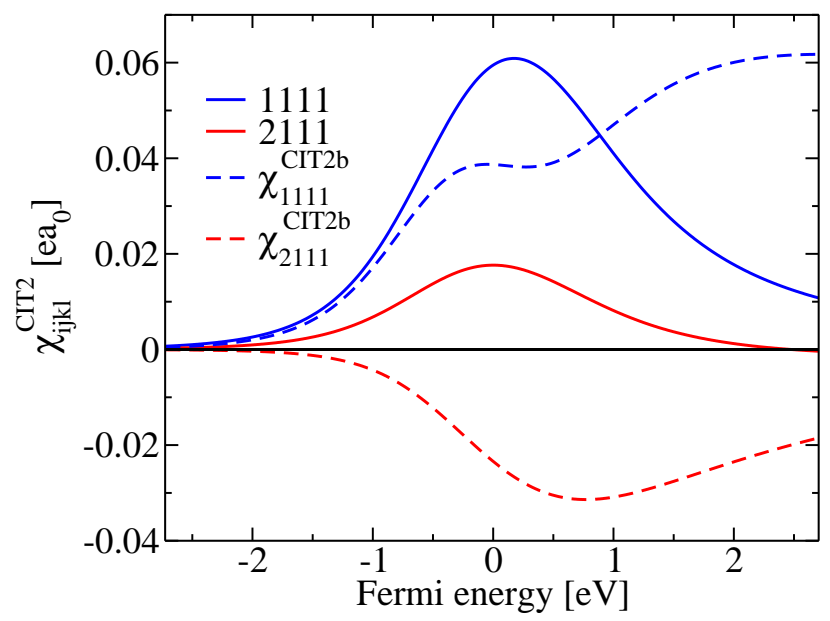

FIG. 5: Chiral CIT for helical gradients in the 1d Rashba model vs. Fermi energy. Dashed lines: Contributions from CIDMI.

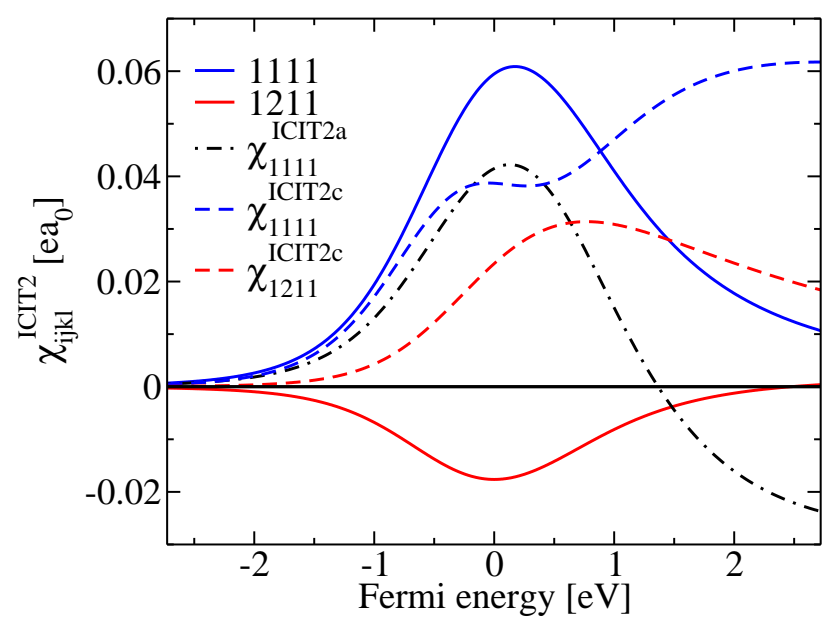

FIG. 6: Chiral ICIT for helical gradients in the 1d Rashba model vs. Fermi energy. Dashed lines: Contributions from DOM. Dashed-dotted line: Contribution from the timedependent magnetization gradient.

magnetization direction varies with the $x$ coordinate.

In Fig. 9 and Fig. 10 we show the chiral CIT and ICIT, respectively, for helical gradients in the $2 \mathrm{~d}$ Rashba model. The component $\chi_{2111}^{\mathrm{CIT} 2}$ describes the adiabatic STT with SOI and the component $\chi_{1111}^{\mathrm{CIT} 2}$ describes the non-adiabatic STT. The components $\chi_{2211}^{\mathrm{CIT} 2}$ and $\chi_{1211}^{\mathrm{CIT} 2}$ describe the case when the applied electric field points in $y$ direction, i.e., perpendicular to the direction along which the magnetization direction varies. Comparison between Fig. 9 and Fig. 10 shows that $\chi_{1111}^{\mathrm{CIT} 2}$ and $\chi_{2211}^{\mathrm{CIT} 2}$ are even in $\hat{\boldsymbol{M}}$, that $\chi_{1211}^{\mathrm{CIT} 2}$ and $\chi_{2111}^{\mathrm{CIT} 2}$ are odd in $\hat{\boldsymbol{M}}$ and that CIT and ICIT satisfy the reciprocity relation Eq. (5) when the gradients of CIDMI and DOM are included, i.e., 


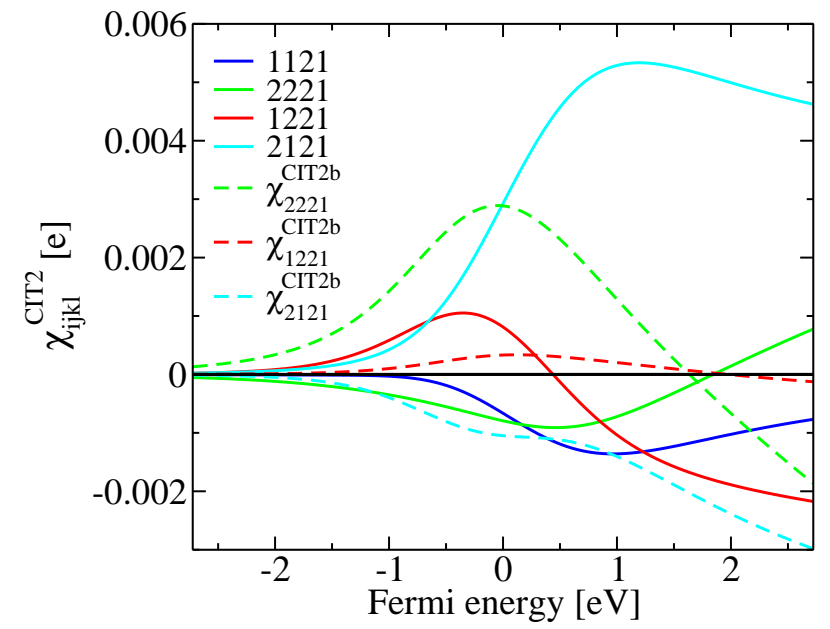

FIG. 7: Chiral CIT for cycloidal gradients in the 2d Rashba model vs. Fermi energy. Dashed lines: Contributions from CIDMI.

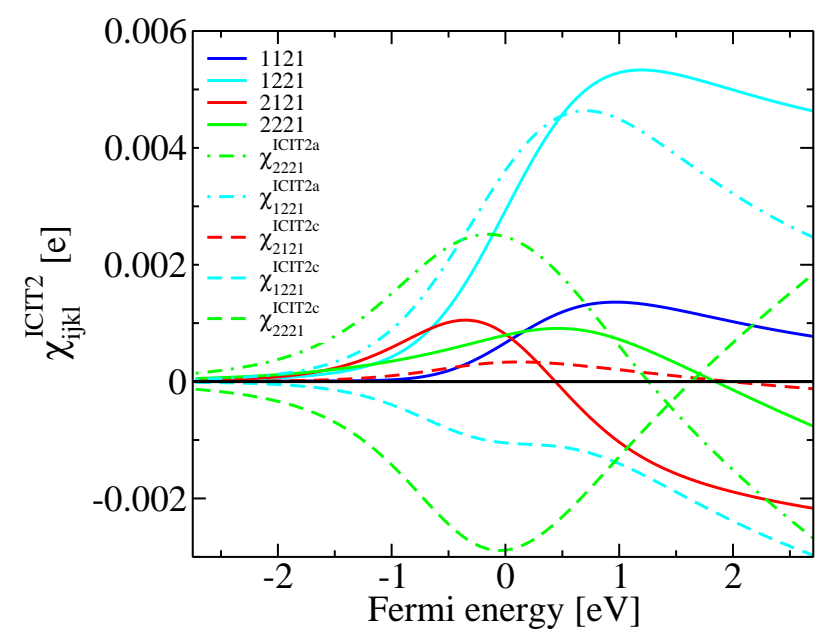

FIG. 8: Chiral ICIT for cycloidal gradients in the 2d Rashba model vs. Fermi energy. Dashed lines: Contributions from DOM. Dashed-dotted lines: Contributions from the timedependent gradients.

$\chi_{1111}^{\mathrm{CIT} 2}=\chi_{1111}^{\mathrm{ICIT2}}, \chi_{2211}^{\mathrm{CIT} 2}=\chi_{2211}^{\mathrm{ICIT2} 2}, \chi_{1211}^{\mathrm{IIT} 2}=-\chi_{2111}^{\mathrm{ICIT2}}$, and $\chi_{2111}^{\mathrm{CIT} 2}=-\chi_{1211}^{\mathrm{ICIT} 2}$.

\section{B. Chiral torque-torque correlation}

In Fig. 11 we show the chiral contribution to the torque-torque correlation in the 1d Rashba model for cycloidal gradients. We compare the perturbation theory Eq. (78) plus Eq. (82) to the gauge-field approach from Ref. [38]. This comparison shows that perturbation theory provides the correct answer only when the contribution $\chi_{i j k l}^{\mathrm{TT} 2 \mathrm{a}}(\mathrm{Eq}$. (82) ) from the time-dependent

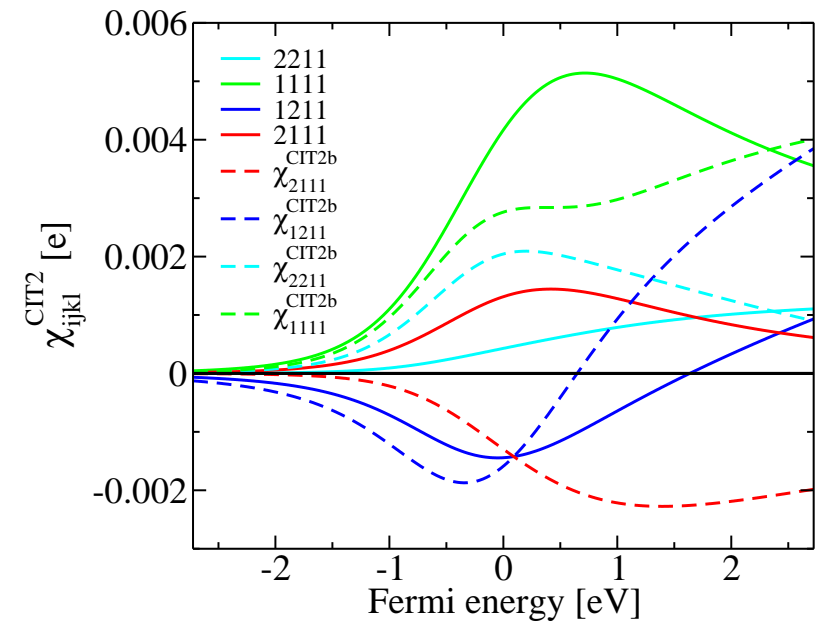

FIG. 9: Chiral CIT for helical gradients in the 2d Rashba model vs. Fermi energy. Dashed lines: Contributions from CIDMI.

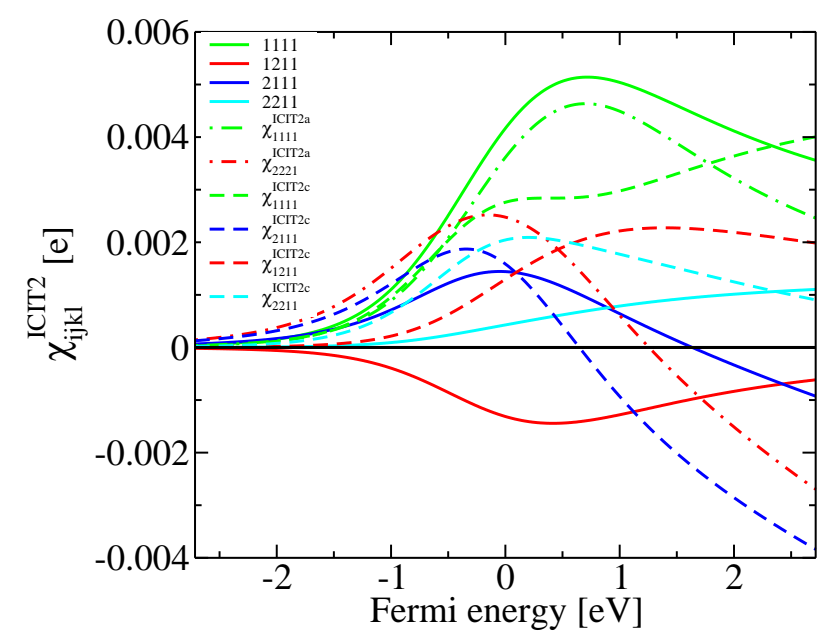

FIG. 10: Chiral ICIT for helical gradients in the 2d Rashba model vs. Fermi energy. Dashed lines: Contributions from DOM. Dashed-dotted lines: Contributions from the timedependent gradient.

gradients is taken into account. The contributions $\chi_{1221}^{\mathrm{TT} 2 \mathrm{a}}$ and $\chi_{2221}^{\mathrm{TT} 2 \mathrm{a}}$ from the time-dependent gradients are comparable in magnitude to the total values. In the 1d Rashba model the DDMI-contribution in Eq. (60) is zero for cycloidal gradients (not shown in the figure). The components $\chi_{2121}^{\text {TT2 }}$ and $\chi_{1221}^{\text {TT2 }}$ describe the chiral gyromagnetism while the components $\chi_{1121}^{\mathrm{TT} 2}$ and $\chi_{2221}^{\mathrm{TT} 2}$ describe the chiral damping [38, 40, 41]. The components $\chi_{2121}^{\mathrm{TT} 2}$ and $\chi_{1221}^{\mathrm{TT} 2}$ are odd in $\hat{\boldsymbol{M}}$ and they satisfy the Onsager relation Eq. (58), i.e., $\chi_{2121}^{\mathrm{TT} 2}=-\chi_{1221}^{\mathrm{TT} 2}$.

In Fig. 12 we show the chiral contributions to the torque-torque correlation in the 1d Rashba model for helical gradients. In contrast to the cycloidal gradients 


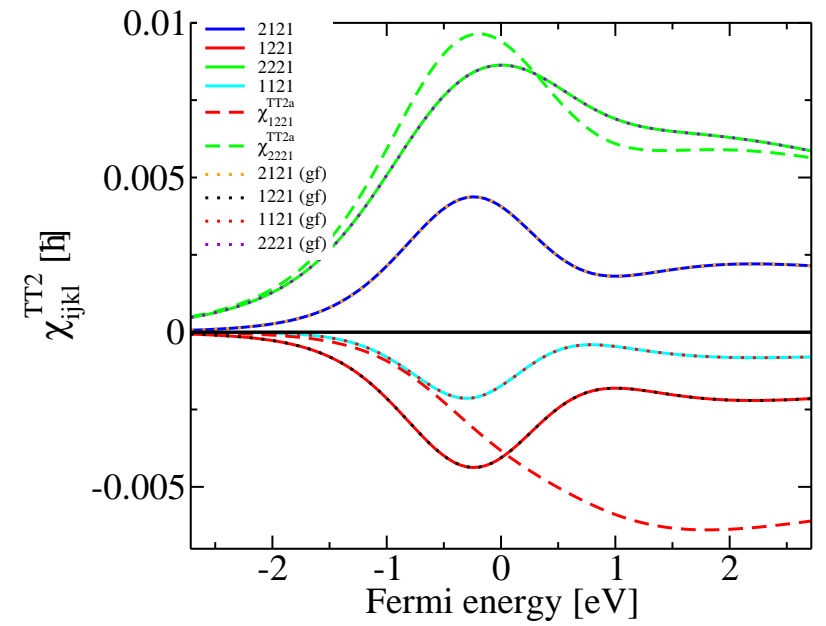

FIG. 11: Chiral contribution to the torque-torque correlation for cycloidal gradients in the 1d Rashba model vs. Fermi energy. Perturbation theory (solid lines) agrees to the gaugefield (gf) approach (dotted lines). Dashed lines: Contribution from the time-dependent gradient.

(Fig. 11) there are contributions from the spatial gradients of DDMI (Eq. (60) in this case. The Onsager relation Eq. (58) for the components $\chi_{2111}^{\mathrm{TT} 2}$ and $\chi_{1211}^{\mathrm{TT} 2}$ is satisfied only when these contributions from DDMI are taken into account, which are of the same order of magnitude as the total values. The components $\chi_{2111}^{\mathrm{TT} 2}$ and $\chi_{1211}^{\mathrm{TT} 2}$ are even in $\hat{\boldsymbol{M}}$ and describe chiral damping, while the components $\chi_{1111}^{\mathrm{TT} 2}$ and $\chi_{2211}^{\mathrm{TT} 2}$ are odd in $\hat{\boldsymbol{M}}$ and describe chiral gyromagnetism. As a consequence of the Onsager relation Eq. (58) we obtain $\chi_{1111}^{\mathrm{TT} 2}=\chi_{2211}^{\mathrm{TT} 2}=0$ for the total components: Eq. (58) shows that diagonal components of the torque-torque correlation function are zero unless they are even in $\hat{\boldsymbol{M}}$. However, $\chi_{1111}^{\mathrm{TT} 2 \mathrm{a}}, \chi_{1111}^{\mathrm{TT} 2 \mathrm{c}}$, and $\chi_{1111}^{\mathrm{TT} 2 \mathrm{~b}}=-\chi_{1111}^{\mathrm{TT} 2 \mathrm{a}}-\chi_{1111}^{\mathrm{TT} 2 \mathrm{c}}$ are individually nonzero. Interestingly, the off-diagonal components of the torquetorque correlation describe chiral damping for helical gradients, while for cycloidal gradients the off-diagonal elements describe chiral gyromagnetism and the diagonal elements describe chiral damping.

In Fig. 13 we show the chiral contributions to the torque-torque correlation in the $2 \mathrm{~d}$ Rashba model for cycloidal gradients. In contrast to the $1 \mathrm{~d}$ Rashba model with cycloidal gradients (Fig. 111) the contributions from DDMI $\chi_{i j k l}^{\mathrm{TT} 2 \mathrm{c}}(\mathrm{Eq} .(600)$ are nonzero in this case. Without these contributions from DDMI the Onsager relation (58) $\chi_{2121}^{\mathrm{TT} 2}=-\chi_{1221}^{\mathrm{TT} 2}$ is violated. The DDMI contribution is of the same order of magnitude as the total values. The components $\chi_{2121}^{\mathrm{TT} 2}$ and $\chi_{1221}^{\mathrm{TT} 2}$ are odd in $\hat{\boldsymbol{M}}$ and describe chiral gyromagnetism, while the components $\chi_{1121}^{\mathrm{TT} 2}$ and $\chi_{2221}^{\mathrm{TT} 2}$ are even in $\hat{\boldsymbol{M}}$ and describe chiral damping.

In Fig. 14 we show the chiral contributions to the torque-torque correlation in the $2 \mathrm{~d}$ Rashba model for helical gradients. The components $\chi_{1211}^{\mathrm{TT} 2}$ and $\chi_{2111}^{\mathrm{TT} 2}$ are even

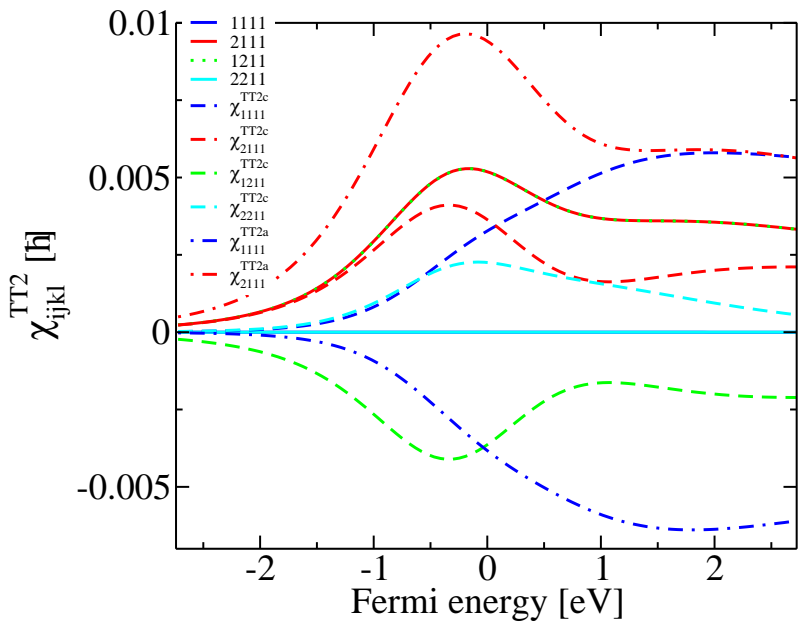

FIG. 12: Chiral contribution to the torque-torque correlation for helical gradients in the 1d Rashba model vs. Fermi energy. Dashed lines: Contributions from DDMI. Dasheddotted lines: Contributions from the time-dependent gradients.

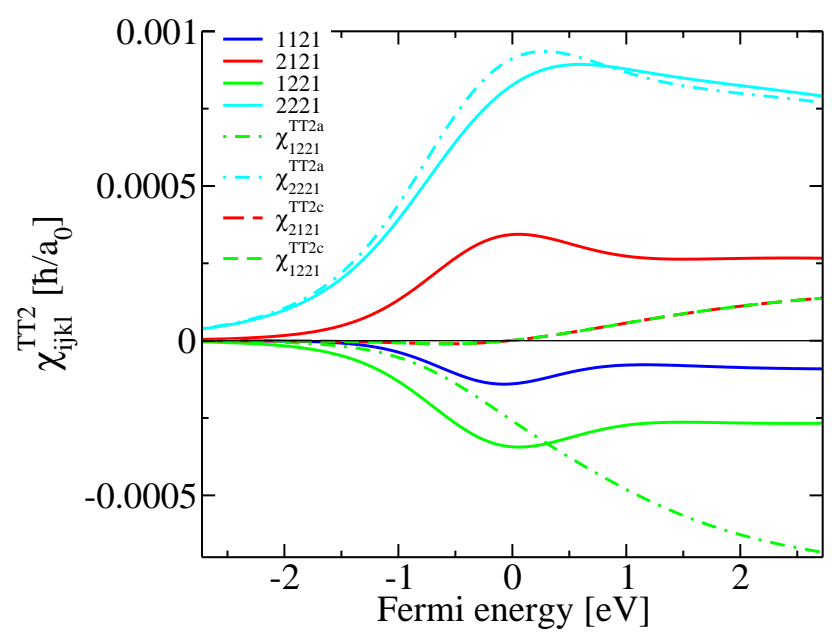

FIG. 13: Chiral contribution to the torque-torque correlation for cycloidal gradients in the 2d Rashba model vs. Fermi energy. Dashed lines: Contributions from DDMI. Dasheddotted lines: Contributions from the time-dependent gradients.

in $\hat{\boldsymbol{M}}$ and describe chiral damping, while the components $\chi_{1111}^{\mathrm{TT} 2}$ and $\chi_{2211}^{\mathrm{TT} 2}$ are odd in $\hat{\boldsymbol{M}}$ and describe chiral gyromagnetism. The Onsager relation Eq. (58) requires $\chi_{1111}^{\mathrm{TT} 2}=\chi_{2211}^{\mathrm{TT} 2}=0$ and $\chi_{2111}^{\mathrm{TT} 2}=\chi_{1211}^{\mathrm{TT} 2}$. Without the contributions from DDMI these Onsager relations are violated. 


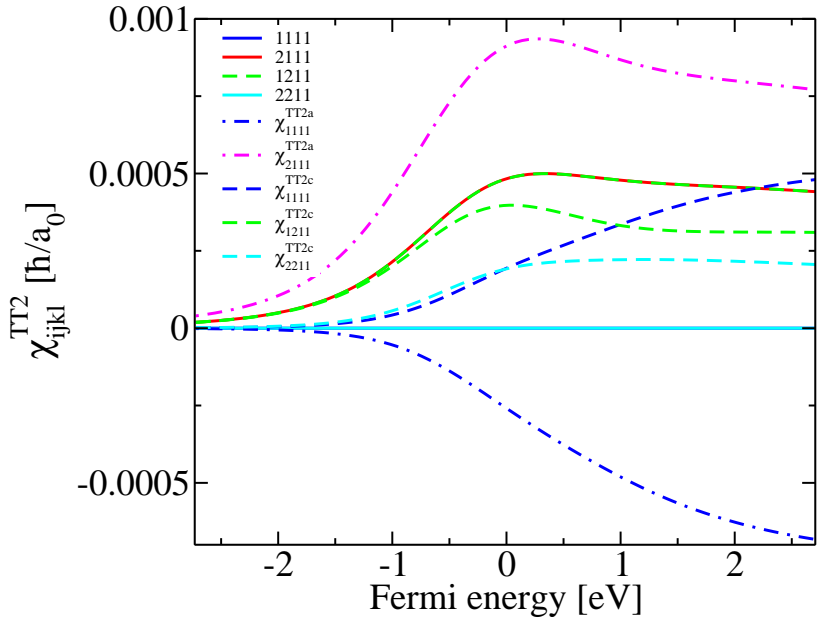

FIG. 14: Chiral contribution to the torque-torque correlation for helical gradients in the $2 \mathrm{~d}$ Rashba model vs. Fermi energy. Dashed lines: Contributions from DDMI. Dasheddotted lines: Contributions from the time-dependent gradients.

\section{SUMMARY}

Finding ways to tune the Dzyaloshinskii-Moriya interaction (DMI) by external means, such as an applied electric current, holds much promise for applications in which DMI determines the magnetic texture of domain walls or skyrmions. In order to derive an expression for currentinduced Dzyaloshinskii-Moriya interaction (CIDMI) we first identify its inverse effect: When magnetic textures vary as a function of time, electric currents are driven by various mechanisms, which can be distinguished according to their different dependence on the time-derivative of magnetization, $\partial \hat{\boldsymbol{M}}(\boldsymbol{r}, t) / \partial t$, and on the spatial derivative $\partial \hat{\boldsymbol{M}}(\boldsymbol{r}, t) / \partial \boldsymbol{r}$ : One group of effects is proportional to $\partial \hat{\boldsymbol{M}}(\boldsymbol{r}, t) / \partial t$, a second group of effects is proportional to the product $\partial \hat{\boldsymbol{M}}(\boldsymbol{r}, t) / \partial t \partial \hat{\boldsymbol{M}}(\boldsymbol{r}, t) / \partial \boldsymbol{r}$, and a third group is proportional to the second derivative $\partial^{2} \hat{\boldsymbol{M}}(\boldsymbol{r}, t) / \partial \boldsymbol{r} \partial t$. We show that the response of the electric current to the time-dependent magnetization gradient $\partial^{2} \hat{\boldsymbol{M}}(\boldsymbol{r}, t) / \partial \boldsymbol{r} \partial t$ contais the inverse of CIDMI. We establish the reciprocity relation between inverse and direct CIDMI and thereby obtain an expression for CIDMI. We find that CIDMI is related to the modification of orbital magnetism induced by magnetization dynamics, which we call dynamical orbital magnetism (DOM). We show that torques are generated by time-dependent gradients of magnetization as well. The inverse effect consists in the modification of DMI by magnetization dynamics, which we call dynamical DMI (DDMI).

Additionally, we develop a formalism to calculate the chiral contributions to the direct and inverse currentinduced torques (CITs) and to the torque-torque correla- tion in noncollinear magnets. We show that the response to time-dependent magnetization gradients contributes substantially to these effects and that the Onsager reciprocity relations are violated when it is not taken into account. In noncollinear magnets CIDMI, DDMI and DOM depend on the local magnetization direction. We show that the resulting spatial gradients of CIDMI, DDMI and DOM have to be subtracted from the CIT, from the torque-torque correlation, and from the inverse CIT, respectively.

We apply our formalism to study CITs and the torquetorque correlation in textured Rashba ferromagnets. We find that the contribution of CIDMI to the chiral CIT is of the order of magnitude of the total effect. Similarly, we find that the contribution of DDMI to the chiral torquetorque correlation is of the order of magnitude of the total effect.

\section{Acknowledgments}

We acknowledge financial support from Leibniz Collaborative Excellence project OptiSPIN - Optical Control of Nanoscale Spin Textures. We acknowledge funding under SPP 2137 "Skyrmionics" of the DFG. We gratefully acknowledge financial support from the European Research Council (ERC) under the European Union's Horizon 2020 research and innovation program (Grant No. 856538, project "3D MAGiC"). The work was also supported by the Deutsche Forschungsgemeinschaft (DFG, German Research Foundation) - TRR 173 - 268565370 (project A11). We gratefully acknowledge the Jülich Supercomputing Centre and RWTH Aachen University for providing computational resources under project No. jiff40.

\section{Appendix A: Response to time-dependent gradients}

In this appendix we derive Eq. (18), Eq. (20), Eq. (41), and Eq. (82), which describe the response to timedependent magnetization gradients, and Eq. (50), which describes the response to time-dependent magnetic fields. We consider perturbations of the form

$$
\delta H(\boldsymbol{r}, t)=\mathfrak{B} b \frac{1}{q \omega} \sin (\boldsymbol{q} \cdot \boldsymbol{r}) \sin (\omega t) .
$$

When we set $\mathfrak{B}=\frac{\partial H}{\partial \hat{M}_{k}}$ and $b=\frac{\partial^{2} \hat{M}_{k}}{\partial r_{i} \partial t}$, Eq. (A1) turns into Eq. (17), while when we set $\mathfrak{B}=-e v_{i}$ and $b=\frac{1}{2} \epsilon_{i j k} \frac{\partial B_{k}}{\partial t}$ we obtain Eq. (48). We need to derive an expression for the response $\delta A(\boldsymbol{r}, t)$ of an observable $\mathfrak{A}$ to this perturbation, which varies in time like $\cos (\omega t)$ and in space like $\cos (\boldsymbol{q} \cdot \boldsymbol{r})$, because $\frac{\partial^{2} \hat{M}(\boldsymbol{r}, t)}{\partial r_{i} \partial t} \propto \cos (\boldsymbol{q} \cdot \boldsymbol{r}) \cos (\omega t)$. Therefore, we use the Kubo linear response formalism to obtain 
the coefficient $\chi$ in

$$
\delta A(\boldsymbol{r}, t)=\chi \cos (\boldsymbol{q} \cdot \boldsymbol{r}) \cos (\omega t),
$$

which is given by

$$
\begin{aligned}
& \chi=\frac{i}{\hbar q \omega V} {\left[\langle\langle\mathfrak{A} \cos (\boldsymbol{q} \cdot \boldsymbol{r}), \mathfrak{B} \sin (\boldsymbol{q} \cdot \boldsymbol{r})\rangle\rangle^{\mathrm{R}}(\hbar \omega)\right.} \\
&\left.-\langle\langle\mathfrak{A} \cos (\boldsymbol{q} \cdot \boldsymbol{r}), \mathfrak{B} \sin (\boldsymbol{q} \cdot \boldsymbol{r})\rangle\rangle^{\mathrm{R}}(-\hbar \omega)\right],
\end{aligned}
$$

where $\langle\langle\mathfrak{A} \cos (\boldsymbol{q} \cdot \boldsymbol{r}), \mathfrak{B} \sin (\boldsymbol{q} \cdot \boldsymbol{r})\rangle\rangle^{\mathrm{R}}(\hbar \omega)$ is the retarded function at frequency $\omega$ and $V$ is the volume of the unit cell.

The operator $\mathfrak{B} \sin (\boldsymbol{q} \cdot \boldsymbol{r})$ can be written as

$\mathfrak{B} \sin (\boldsymbol{q} \cdot \boldsymbol{r})=\frac{1}{2 i} \sum_{\boldsymbol{k n m}}\left[\mathfrak{B}_{\boldsymbol{k n m}}^{(1)} c_{\boldsymbol{k}_{+} n}^{\dagger} c_{\boldsymbol{k}_{-} m}-\mathfrak{B}_{\boldsymbol{k} n m}^{(2)} c_{\boldsymbol{k}_{-} n}^{\dagger} c_{\boldsymbol{k}_{+} m}\right]$,

where $\boldsymbol{k}_{+}=\boldsymbol{k}+\boldsymbol{q} / 2, \boldsymbol{k}_{-}=\boldsymbol{k}-\boldsymbol{q} / 2, c_{\boldsymbol{k}_{+} n}^{\dagger}$ is the creation operator of an electron in state $\left|u_{\boldsymbol{k}_{+} n}\right\rangle, c_{\boldsymbol{k}_{-} m}$ is the annihilation operator of an electron in state $\left|u_{\boldsymbol{k}_{-} m}\right\rangle$,

$$
\mathfrak{B}_{\boldsymbol{k} n m}^{(1)}=\frac{1}{2}\left\langle u_{\boldsymbol{k}_{+} n}\left|\left[\mathfrak{B}_{\boldsymbol{k}_{+}}+\mathfrak{B}_{\boldsymbol{k}_{-}}\right]\right| u_{\boldsymbol{k}_{-}}\right\rangle
$$

and

$$
\mathfrak{B}_{\boldsymbol{k} n m}^{(2)}=\frac{1}{2}\left\langle u_{\boldsymbol{k}_{-} n}\left|\left[\mathfrak{B}_{\boldsymbol{k}_{+}}+\mathfrak{B}_{\boldsymbol{k}_{-}}\right]\right| u_{\boldsymbol{k}_{+} m}\right\rangle .
$$

Similarly,

$\mathfrak{A} \cos (\boldsymbol{q} \cdot \boldsymbol{r})=\frac{1}{2} \sum_{\boldsymbol{k n m}}\left[\mathfrak{A}_{\boldsymbol{k n m}}^{(1)} c_{\boldsymbol{k}_{+} n}^{\dagger} c_{\boldsymbol{k}_{-m}}+\mathfrak{A}_{\boldsymbol{k n m}}^{(2)} c_{\boldsymbol{k}_{-} n}^{\dagger} c_{\boldsymbol{k}_{+} m}\right]$,

where

$$
\mathfrak{A}_{\boldsymbol{k} n m}^{(1)}=\frac{1}{2}\left\langle u_{\boldsymbol{k}_{+} n}\left|\left[\mathfrak{A}_{\boldsymbol{k}_{+}}+\mathfrak{A}_{\boldsymbol{k}_{-}}\right]\right| u_{\boldsymbol{k}_{-}}\right\rangle
$$

and

$$
\mathfrak{A}_{\boldsymbol{k} n m}^{(2)}=\frac{1}{2}\left\langle u_{\boldsymbol{k}_{-} n}\left|\left[\mathfrak{A}_{\boldsymbol{k}_{+}}+\mathfrak{A}_{\boldsymbol{k}_{-}}\right]\right| u_{\boldsymbol{k}_{+} m}\right\rangle .
$$

It is convenient to obtain the retarded response function in Eq. (A3) from the corresponding Matsubara function in imaginary time $\tau$

$$
\begin{aligned}
& \frac{1}{V}\langle\langle\mathfrak{A} \cos (\boldsymbol{q} \cdot \boldsymbol{r}), \mathfrak{B} \sin (\boldsymbol{q} \cdot \boldsymbol{r})\rangle\rangle^{\mathrm{M}}(\tau)= \\
& =\frac{1}{4 i} \int \frac{\mathrm{d}^{d} k}{(2 \pi)^{d}} \sum_{n m} \sum_{n^{\prime} m^{\prime}}\left[\mathfrak{A}_{\boldsymbol{k} n m^{\prime}}^{(1)} \mathfrak{B}_{\boldsymbol{k} n^{\prime} m^{\prime}}^{(2)} \mathscr{Z}_{\boldsymbol{k} n m n^{\prime} m^{\prime}}^{(1)}(\tau)\right. \\
& \left.-\mathfrak{A}_{\boldsymbol{k} n m}^{(2)} \mathfrak{B}_{\boldsymbol{k} n^{\prime} m^{\prime}}^{(1)} \mathscr{Z}_{\boldsymbol{k} n m n^{\prime} m^{\prime}}^{(2)}(\tau)\right],
\end{aligned}
$$

where $d=1,2$ or 3 is the dimension,

$$
\begin{aligned}
\mathscr{Z}_{\boldsymbol{k} n m n^{\prime} m^{\prime}}^{(1)}(\tau) & =\left\langle T_{\tau} c_{\boldsymbol{k}_{+} n}^{\dagger}(\tau) c_{\boldsymbol{k}_{-} m}(\tau) c_{\boldsymbol{k}_{-} n^{\prime}}^{\dagger}(0) c_{\boldsymbol{k}_{+} m^{\prime}}(0)\right\rangle \\
& =-G_{m^{\prime} n}^{\mathrm{M}}\left(\boldsymbol{k}_{+},-\tau\right) G_{m n^{\prime}}^{\mathrm{M}}\left(\boldsymbol{k}_{-}, \tau\right)
\end{aligned}
$$

$$
\begin{aligned}
\mathscr{Z}_{\boldsymbol{k} n m n^{\prime} m^{\prime}}^{(2)}(\tau) & =\left\langle T_{\tau} c_{\boldsymbol{k}_{-} n}^{\dagger}(\tau) c_{\boldsymbol{k}_{+} m}(\tau) c_{\boldsymbol{k}_{+} n^{\prime}}^{\dagger}(0) c_{\boldsymbol{k}_{-} m^{\prime}}(0)\right\rangle \\
& =-G_{m^{\prime} n}^{\mathrm{M}}\left(\boldsymbol{k}_{-},-\tau\right) G_{m n^{\prime}}^{\mathrm{M}}\left(\boldsymbol{k}_{+}, \tau\right),
\end{aligned}
$$

and

$$
G_{m n^{\prime}}^{\mathrm{M}}\left(\boldsymbol{k}_{+}, \tau\right)=-\left\langle T_{\tau} c_{\boldsymbol{k}_{+} m}(\tau) c_{\boldsymbol{k}_{+} n^{\prime}}^{\dagger}(0)\right\rangle
$$

is the single-particle Matsubara function. The Fourier transform of Eq. A10 is given by

$$
\begin{aligned}
& \frac{1}{V}\langle\langle\mathfrak{A} \cos (\boldsymbol{q} \cdot \boldsymbol{r}), \mathfrak{B} \sin (\boldsymbol{q} \cdot \boldsymbol{r})\rangle\rangle^{\mathrm{M}}\left(i \mathcal{E}_{N}\right)= \\
& =\frac{i}{4 \hbar \beta} \int \frac{\mathrm{d}^{d} k}{(2 \pi)^{d}} \sum_{n m} \sum_{n^{\prime} m^{\prime}} \sum_{p}[ \\
& \mathfrak{A}_{\boldsymbol{k} n m}^{(1)} \mathfrak{B}_{\boldsymbol{k} n^{\prime} m^{\prime}}^{(2)} G_{m^{\prime} n}^{\mathrm{M}}\left(\boldsymbol{k}_{+}, i \mathcal{E}_{p}\right) G_{m n^{\prime}}^{\mathrm{M}}\left(\boldsymbol{k}_{-}, i \mathcal{E}_{p}+i \mathcal{E}_{N}\right) \\
& \left.-\mathfrak{A}_{\boldsymbol{k} n m}^{(2)} \mathfrak{B}_{\boldsymbol{k} n^{\prime} m^{\prime}}^{(1)} G_{m^{\prime} n}^{\mathrm{M}}\left(\boldsymbol{k}_{-}, i \mathcal{E}_{p}\right) G_{m n^{\prime}}^{\mathrm{M}}\left(\boldsymbol{k}_{+}, i \mathcal{E}_{p}+i \mathcal{E}_{N}\right)\right],
\end{aligned}
$$

where $\mathcal{E}_{N}=2 \pi N / \beta$ and $\mathcal{E}_{p}=(2 p+1) \pi / \beta$ are bosonic and fermionic Matsubara energy points, respectively, and $\beta=1 /\left(k_{\mathrm{B}} T\right)$ is the inverse temperature.

In order to carry out the Matsubara summation over $\mathcal{E}_{p}$ we make use of

$$
\begin{aligned}
& \frac{1}{\beta} \sum_{p} G_{m n^{\prime}}^{\mathrm{M}}\left(i \mathcal{E}_{p}+i \mathcal{E}_{N}\right) G_{m^{\prime} n}^{\mathrm{M}}\left(i \mathcal{E}_{p}\right)= \\
& =\frac{i}{2 \pi} \int d \mathcal{E}^{\prime} f\left(\mathcal{E}^{\prime}\right) G_{m n^{\prime}}^{\mathrm{M}}\left(\mathcal{E}^{\prime}+i \mathcal{E}_{N}\right) G_{m^{\prime} n}^{\mathrm{M}}\left(\mathcal{E}^{\prime}+i \delta\right) \\
& +\frac{i}{2 \pi} \int d \mathcal{E}^{\prime} f\left(\mathcal{E}^{\prime}\right) G_{m n^{\prime}}^{\mathrm{M}}\left(\mathcal{E}^{\prime}+i \delta\right) G_{m^{\prime} n}^{\mathrm{M}}\left(\mathcal{E}^{\prime}-i \mathcal{E}_{N}\right) \\
& -\frac{i}{2 \pi} \int d \mathcal{E}^{\prime} f\left(\mathcal{E}^{\prime}\right) G_{m n^{\prime}}^{\mathrm{M}}\left(\mathcal{E}^{\prime}+i \mathcal{E}_{N}\right) G_{m^{\prime} n}^{\mathrm{M}}\left(\mathcal{E}^{\prime}-i \delta\right) \\
& -\frac{i}{2 \pi} \int d \mathcal{E}^{\prime} f\left(\mathcal{E}^{\prime}\right) G_{m n^{\prime}}^{\mathrm{M}}\left(\mathcal{E}^{\prime}-i \delta\right) G_{m^{\prime} n}^{\mathrm{M}}\left(\mathcal{E}^{\prime}-i \mathcal{E}_{N}\right),
\end{aligned}
$$

where $\delta$ is a positive infinitesimal. The retarded function $\langle\langle\mathfrak{A} \cos (\boldsymbol{q} \cdot \boldsymbol{r}), \mathfrak{B} \sin (\boldsymbol{q} \cdot \boldsymbol{r})\rangle\rangle^{\mathrm{R}}(\omega)$ is obtained from the Matsubara function $\langle\langle\mathfrak{A} \cos (\boldsymbol{q} \cdot \boldsymbol{r}), \mathfrak{B} \sin (\boldsymbol{q} \cdot \boldsymbol{r})\rangle\rangle^{\mathrm{M}}\left(i \mathcal{E}_{N}\right)$ by the analytic continuation $i \mathcal{E}_{N} \rightarrow \hbar \omega$ to real frequencies. The right-hand side of Eq. A15 has the following analytic continuation to real frequencies:

$$
\begin{gathered}
\frac{i}{2 \pi} \int d \mathcal{E}^{\prime} f\left(\mathcal{E}^{\prime}\right) G_{m n^{\prime}}^{\mathrm{R}}\left(\mathcal{E}^{\prime}+\hbar \omega\right) G_{m^{\prime} n}^{\mathrm{R}}\left(\mathcal{E}^{\prime}\right) \\
+\frac{i}{2 \pi} \int d \mathcal{E}^{\prime} f\left(\mathcal{E}^{\prime}\right) G_{m n^{\prime}}^{\mathrm{R}}\left(\mathcal{E}^{\prime}\right) G_{m^{\prime} n}^{\mathrm{A}}\left(\mathcal{E}^{\prime}-\hbar \omega\right) \\
-\frac{i}{2 \pi} \int d \mathcal{E}^{\prime} f\left(\mathcal{E}^{\prime}\right) G_{m n^{\prime}}^{\mathrm{R}}\left(\mathcal{E}^{\prime}+\hbar \omega\right) G_{m^{\prime} n}^{\mathrm{A}}\left(\mathcal{E}^{\prime}\right) \\
-\frac{i}{2 \pi} \int d \mathcal{E}^{\prime} f\left(\mathcal{E}^{\prime}\right) G_{m n^{\prime}}^{\mathrm{A}}\left(\mathcal{E}^{\prime}\right) G_{m^{\prime} n}^{\mathrm{A}}\left(\mathcal{E}^{\prime}-\hbar \omega\right) .
\end{gathered}
$$

Therefore, we obtain

$$
\begin{aligned}
& \chi=\frac{-i}{8 \pi \hbar^{2} q \omega} \int \frac{\mathrm{d}^{d} k}{(2 \pi)^{d}} {\left[Z_{\boldsymbol{k}}(q, \omega)-Z_{\boldsymbol{k}}(-q, \omega)\right.} \\
&\left.-Z_{\boldsymbol{k}}(q,-\omega)+Z_{\boldsymbol{k}}(-q,-\omega)\right],
\end{aligned}
$$


where

$$
\begin{aligned}
& Z_{\boldsymbol{k}}(q, \omega)= \\
& =\int d \mathcal{E}^{\prime} f\left(\mathcal{E}^{\prime}\right) \operatorname{Tr}\left[\mathfrak{A}_{\boldsymbol{k}} G_{\boldsymbol{k}_{-}}^{\mathrm{R}}\left(\mathcal{E}^{\prime}+\hbar \omega\right) \mathfrak{B}_{k} G_{\boldsymbol{k}_{+}}^{\mathrm{R}}\left(\mathcal{E}^{\prime}\right)\right] \\
& +\int d \mathcal{E}^{\prime} f\left(\mathcal{E}^{\prime}\right) \operatorname{Tr}\left[\mathfrak{A}_{\boldsymbol{k}} G_{\boldsymbol{k}_{-}}^{\mathrm{R}}\left(\mathcal{E}^{\prime}\right) \mathfrak{B}_{\boldsymbol{k}} G_{\boldsymbol{k}_{+}}^{\mathrm{A}}\left(\mathcal{E}^{\prime}-\hbar \omega\right)\right] \\
& -\int d \mathcal{E}^{\prime} f\left(\mathcal{E}^{\prime}\right) \operatorname{Tr}\left[\mathfrak{A}_{\boldsymbol{k}} G_{\boldsymbol{k}_{-}}^{\mathrm{R}}\left(\mathcal{E}^{\prime}+\hbar \omega\right) \mathfrak{B}_{\boldsymbol{k}} G_{\boldsymbol{k}_{+}}^{\mathrm{A}}\left(\mathcal{E}^{\prime}\right)\right] \\
& -\int d \mathcal{E}^{\prime} f\left(\mathcal{E}^{\prime}\right) \operatorname{Tr}\left[\mathfrak{A}_{\boldsymbol{k}} G_{\boldsymbol{k}_{-}}^{\mathrm{A}}\left(\mathcal{E}^{\prime}\right) \mathfrak{B}_{\boldsymbol{k}} G_{\boldsymbol{k}_{+}}^{\mathrm{A}}\left(\mathcal{E}^{\prime}-\hbar \omega\right)\right] .
\end{aligned}
$$

We consider the limit $\lim _{q \rightarrow 0} \lim _{\omega \rightarrow 0} \chi$. In this limit Eq. (A17) may be rewritten as

$$
\chi=\left.\frac{-i}{2 \pi \hbar^{2}} \int \frac{\mathrm{d}^{d} k}{(2 \pi)^{d}} \frac{\partial^{2} Z_{\boldsymbol{k}}(q, \omega)}{\partial q \partial \omega}\right|_{q=\omega=0} .
$$

The frequency derivative of $Z_{\boldsymbol{k}}(q, \omega)$ is given by

$$
\begin{aligned}
\left.\frac{1}{\hbar} \frac{\partial Z_{\boldsymbol{k}}}{\partial \omega}\right|_{\omega=0} & =\int d \mathcal{E}^{\prime} f\left(\mathcal{E}^{\prime}\right) \operatorname{Tr}\left[\mathfrak{A}_{k} \frac{\partial G_{\boldsymbol{k}_{-}}^{\mathrm{R}}\left(\mathcal{E}^{\prime}\right)}{\partial \mathcal{E}^{\prime}} \mathfrak{B}_{k} G_{\boldsymbol{k}_{+}}^{\mathrm{R}}\left(\mathcal{E}^{\prime}\right)\right] \\
& -\int d \mathcal{E}^{\prime} f\left(\mathcal{E}^{\prime}\right) \operatorname{Tr}\left[\mathfrak{A}_{k} G_{\boldsymbol{k}_{-}}^{\mathrm{R}}\left(\mathcal{E}^{\prime}\right) \mathfrak{B}_{k} \frac{\partial G_{\boldsymbol{k}_{+}}^{\mathrm{A}}\left(\mathcal{E}^{\prime}\right)}{\partial \mathcal{E}^{\prime}}\right] \\
& -\int d \mathcal{E}^{\prime} f\left(\mathcal{E}^{\prime}\right) \operatorname{Tr}\left[\mathfrak{A}_{k} \frac{\partial G_{\boldsymbol{k}_{-}}^{\mathrm{R}}\left(\mathcal{E}^{\prime}\right)}{\partial \mathcal{E}^{\prime}} \mathfrak{B}_{k} G_{\boldsymbol{k}_{+}}^{\mathrm{A}}\left(\mathcal{E}^{\prime}\right)\right] \\
& +\int d \mathcal{E}^{\prime} f\left(\mathcal{E}^{\prime}\right) \operatorname{Tr}\left[\mathfrak{A}_{k} G_{\boldsymbol{k}_{-}}^{\mathrm{A}}\left(\mathcal{E}^{\prime}\right) \mathfrak{B}_{k} \frac{\partial G_{\boldsymbol{k}_{+}}^{\mathrm{A}}\left(\mathcal{E}^{\prime}\right)}{\partial \mathcal{E}^{\prime}}\right]
\end{aligned}
$$

Using $\partial G^{\mathrm{R}}(\mathcal{E}) / \partial \mathcal{E}=-G^{\mathrm{R}}(\mathcal{E}) G^{\mathrm{R}}(\mathcal{E}) / \hbar$ we obtain

$$
\begin{aligned}
\left.\frac{\partial Z_{\boldsymbol{k}}}{\partial \omega}\right|_{\omega=0}= & -\int d \mathcal{E}^{\prime} f\left(\mathcal{E}^{\prime}\right) \operatorname{Tr}\left[\mathfrak{A}_{\boldsymbol{k}} G_{\boldsymbol{k}_{-}}^{\mathrm{R}} G_{\boldsymbol{k}_{-}}^{\mathrm{R}} \mathfrak{B}_{\boldsymbol{k}} G_{\boldsymbol{k}_{+}}^{\mathrm{R}}\right] \\
& +\int d \mathcal{E}^{\prime} f\left(\mathcal{E}^{\prime}\right) \operatorname{Tr}\left[\mathfrak{A}_{\boldsymbol{k}} G_{\boldsymbol{k}_{-}}^{\mathrm{R}} \mathfrak{B}_{\boldsymbol{k}} G_{\boldsymbol{k}_{+}}^{\mathrm{A}} G_{\boldsymbol{k}_{+}}^{\mathrm{A}}\right] \\
& +\int d \mathcal{E}^{\prime} f\left(\mathcal{E}^{\prime}\right) \operatorname{Tr}\left[\mathfrak{A}_{\boldsymbol{k}} G_{\boldsymbol{k}_{-}}^{\mathrm{R}} G_{\boldsymbol{k}_{-}}^{\mathrm{R}} \mathfrak{B}_{\boldsymbol{k}} G_{\boldsymbol{k}_{+}}^{\mathrm{A}}\right] \\
& -\int d \mathcal{E}^{\prime} f\left(\mathcal{E}^{\prime}\right) \operatorname{Tr}\left[\mathfrak{A}_{\boldsymbol{k}} G_{\boldsymbol{k}_{-}}^{\mathrm{A}} \mathfrak{B}_{\boldsymbol{k}} G_{\boldsymbol{k}_{+}}^{\mathrm{A}} G_{\boldsymbol{k}_{+}}^{\mathrm{A}}\right]
\end{aligned}
$$

Making use of

$$
\lim _{q \rightarrow 0} \frac{\partial G_{\boldsymbol{k}_{+}}^{\mathrm{R}}}{\partial q}=\frac{1}{2} G_{\boldsymbol{k}}^{\mathrm{R}} \frac{\boldsymbol{v} \cdot \boldsymbol{q}}{q} G_{\boldsymbol{k}}^{\mathrm{R}}
$$

we finally obtain

$$
\begin{aligned}
\chi= & \frac{-i}{2 \pi \hbar^{2}} \int \frac{\mathrm{d}^{d} k}{(2 \pi)^{d}} \lim _{q \rightarrow 0} \lim _{\omega \rightarrow 0} \frac{\partial^{2} Z(q, \omega)}{\partial q \partial \omega}= \\
= & \frac{-i}{4 \pi \hbar^{2}} \frac{q}{q} \cdot \int \frac{\mathrm{d}^{d} k}{(2 \pi)^{d}} \int d \mathcal{E} f(\mathcal{E}) \operatorname{Tr}[ \\
& \mathfrak{A}_{\boldsymbol{k}} R \boldsymbol{v} R R \mathfrak{B}_{\boldsymbol{k}} R+\mathfrak{A}_{\boldsymbol{k}} R R \boldsymbol{v} R \mathfrak{B}_{\boldsymbol{k}} R \\
& -\mathfrak{A}_{\boldsymbol{k}} R R \mathfrak{B}_{\boldsymbol{k}} R \boldsymbol{v} R-\mathfrak{A}_{\boldsymbol{k}} R \boldsymbol{v} R \mathfrak{B}_{\boldsymbol{k}} A A \\
& +\mathfrak{A}_{\boldsymbol{k}} R \mathfrak{B}_{\boldsymbol{k}} A \boldsymbol{v} A A+\mathfrak{A}_{\boldsymbol{k}} R \mathfrak{B}_{\boldsymbol{k}} A A \boldsymbol{v} A \\
& -\mathfrak{A}_{\boldsymbol{k}} R \boldsymbol{v} R R \mathfrak{B}_{\boldsymbol{k}} A-\mathfrak{A}_{\boldsymbol{k}} R R \boldsymbol{v} R \mathfrak{B}_{\boldsymbol{k}} A \\
& +\mathfrak{A}_{\boldsymbol{k}} R R \mathfrak{B}_{\boldsymbol{k}} A \boldsymbol{v} A \\
& +\mathfrak{A}_{\boldsymbol{k}} A \boldsymbol{v} A \mathfrak{B}_{\boldsymbol{k}} A A-\mathfrak{A}_{\boldsymbol{k}} A \mathfrak{B}_{\boldsymbol{k}} A \boldsymbol{v} A A \\
& \left.-\mathfrak{A}_{\boldsymbol{k}} A \mathfrak{B}_{\boldsymbol{k}} A A \boldsymbol{v} A\right],
\end{aligned}
$$

where we use the abbreviations $R=G_{\boldsymbol{k}}^{\mathrm{R}}(\mathcal{E})$ and $A=$ $G_{\boldsymbol{k}}^{\mathrm{A}}(\mathcal{E})$. When we substitute $\mathfrak{B}=\frac{\partial H}{\partial \dot{M}_{j}}, \mathfrak{A}=-e v_{i}$, and $\boldsymbol{q}=q_{k} \hat{\boldsymbol{e}}_{k}$, we obtain Eq. (18). When we substitute $\mathfrak{B}=$ $\mathcal{T}_{j}, \mathfrak{A}=-e v_{i}$, and $\boldsymbol{q}=q_{k} \hat{\boldsymbol{e}}_{k}$, we obtain Eq. (20). When we substitute $\mathfrak{A}=-\mathcal{T}_{i}, \mathfrak{B}=\mathcal{T}_{j}$, and $\boldsymbol{q}=q_{k} \hat{\boldsymbol{e}}_{k}$, we obtain Eq. (41). When we substitute $\mathfrak{B}=-e v_{j}, \mathfrak{A}=-\mathcal{T}_{i}$, and $\boldsymbol{q}=q_{k} \hat{\boldsymbol{e}}_{k}$, we obtain Eq. (50). When we substitute $\mathfrak{B}=\frac{\partial H}{\partial \hat{M}_{j}}, \mathfrak{A}=-\mathcal{T}_{i}$, and $\boldsymbol{q}=q_{k} \hat{\boldsymbol{e}}_{k}$, we obtain Eq. (82).

\section{Appendix B: Perturbation theory for the chiral contributions to CIT and to the torque-torque correlation}

In this appendix we derive expressions for the retarded function

$$
\langle\langle\mathfrak{A} \cos (\boldsymbol{q} \cdot \boldsymbol{r}) ; \mathfrak{C}\rangle\rangle^{\mathrm{R}}(\hbar \omega)
$$

within first-order perturbation theory with respect to the perturbation

$$
\delta H=\mathfrak{B} \eta \sin (\boldsymbol{q} \cdot \boldsymbol{r}),
$$

which may arise e.g. from the spatial oscillation of the magnetization direction. As usual, it is convenient to obtain the retarded response function from the corresponding Matsubara function

$$
\langle\langle\cos (\boldsymbol{q} \cdot \boldsymbol{r}) \mathfrak{A} ; \mathfrak{C}\rangle\rangle^{\mathrm{M}}(\tau)=-\left\langle T_{\tau} \cos (\boldsymbol{q} \cdot \boldsymbol{r}) \mathfrak{A}(\tau) \mathfrak{C}(0)\right\rangle .
$$

The starting point for the perturbative expansion is the equation

$$
\begin{aligned}
& -\left\langle T_{\tau} \cos (\boldsymbol{q} \cdot \boldsymbol{r}) \mathfrak{A}\left(\tau_{1}\right) \mathfrak{C}(0)\right\rangle= \\
& =-\frac{\operatorname{Tr}\left[e^{-\beta H} T_{\tau} \cos (\boldsymbol{q} \cdot \boldsymbol{r}) \mathfrak{A}\left(\tau_{1}\right) \mathfrak{C}(0)\right]}{\operatorname{Tr}\left[e^{-\beta H}\right]}= \\
& =-\frac{\operatorname{Tr}\left\{e^{-\beta H_{0}} T_{\tau}\left[U \cos (\boldsymbol{q} \cdot \boldsymbol{r}) \mathfrak{A}\left(\tau_{1}\right) \mathfrak{C}(0)\right]\right\}}{\operatorname{Tr}\left[e^{-\beta H_{0}} U\right]},
\end{aligned}
$$


where $H_{0}$ is the unperturbed Hamiltonian and we consider the first order in the perturbation $\delta H$ :

$$
U^{(1)}=-\frac{1}{\hbar} \int_{0}^{\hbar \beta} d \tau_{1} \mathrm{~T}_{\tau}\left\{e^{\tau_{1} H_{0} / \hbar} \delta H e^{-\tau_{1} H_{0} / \hbar}\right\} .
$$

The essential difference between Eq. (A3) and Eq. (B4) is that in Eq. (A3) the operator $\mathfrak{B}$ enters together with the factor $\sin (\boldsymbol{q} \cdot \boldsymbol{r}) \sin (\omega t)$ (see Eq. (A1)), while in Eq. (B4) only the factor $\sin (\boldsymbol{q} \cdot \boldsymbol{r})$ is connected to $\mathfrak{B}$ in Eq. (B2), while the factor $\sin (\omega t)$ is coupled to the additional operator $\mathfrak{C}$.

We use Eq. (A4) and Eq. (A7) in order to express $\mathfrak{A} \cos (\boldsymbol{q} \cdot \boldsymbol{r})$ and $\mathfrak{B} \sin (\boldsymbol{q} \cdot \boldsymbol{r})$ in terms of annihilation and creation operators. In terms of the correlators

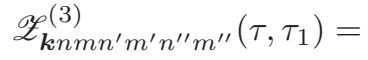

$$
\begin{aligned}
& \left\langle T_{\tau} c_{\boldsymbol{k}_{-} n}^{\dagger}(\tau) c_{\boldsymbol{k}_{+} m}(\tau) c_{\boldsymbol{k}_{+} n^{\prime}}^{\dagger}\left(\tau_{1}\right) c_{\boldsymbol{k}_{-} m^{\prime}}\left(\tau_{1}\right) c_{\boldsymbol{k}_{-} n^{\prime \prime}}^{\dagger} c_{\boldsymbol{k}_{-} m^{\prime \prime}}\right\rangle
\end{aligned}
$$

and

$$
\begin{aligned}
& \mathscr{Z}_{\boldsymbol{k n m n ^ { \prime } m ^ { \prime } n ^ { \prime \prime } m ^ { \prime \prime }}}^{(4)}\left(\tau, \tau_{1}\right)= \\
& \left\langle T_{\tau} c_{\boldsymbol{k}_{-} n}^{\dagger}(\tau) c_{\boldsymbol{k}_{+} m}(\tau) c_{\boldsymbol{k}_{+} n^{\prime}}^{\dagger}\left(\tau_{1}\right) c_{\boldsymbol{k}_{-} m^{\prime}}\left(\tau_{1}\right) c_{\boldsymbol{k}_{+} n^{\prime \prime}}^{\dagger} c_{\boldsymbol{k}_{+} m^{\prime \prime}}\right\rangle
\end{aligned}
$$

and

$$
\begin{aligned}
& \mathscr{Z}_{\boldsymbol{k n m n ^ { \prime } m ^ { \prime } n ^ { \prime \prime } m ^ { \prime \prime }}}^{(5)}\left(\tau, \tau_{1}\right)= \\
& \left\langle T_{\tau} c_{\boldsymbol{k}_{+} n}^{\dagger}(\tau) c_{\boldsymbol{k}_{-} m}(\tau) c_{\boldsymbol{k}_{-} n^{\prime}}^{\dagger}\left(\tau_{1}\right) c_{\boldsymbol{k}_{+} m^{\prime}}\left(\tau_{1}\right) c_{\boldsymbol{k}_{+} n^{\prime \prime}}^{\dagger} c_{\boldsymbol{k}_{+} m^{\prime \prime}}\right\rangle
\end{aligned}
$$

and

$$
\begin{aligned}
& \mathscr{Z}_{\boldsymbol{k n m} m n^{\prime} m^{\prime} n^{\prime \prime} m^{\prime \prime}}^{(6)}\left(\tau, \tau_{1}\right)= \\
& \left\langle T_{\tau} c_{\boldsymbol{k}_{+} n}^{\dagger}(\tau) c_{\boldsymbol{k}_{-} m}(\tau) c_{\boldsymbol{k}_{-} n^{\prime}}^{\dagger}\left(\tau_{1}\right) c_{\boldsymbol{k}_{+} m^{\prime}}\left(\tau_{1}\right) c_{\boldsymbol{k}_{-} n^{\prime \prime}}^{\dagger} c_{\boldsymbol{k}_{-} m^{\prime \prime}}\right\rangle
\end{aligned}
$$

Eq. (B4) can be written as

$$
\begin{aligned}
& \langle\langle\cos (\boldsymbol{q} \cdot \boldsymbol{r}) \mathfrak{A} ; \mathfrak{C}\rangle\rangle^{\mathrm{M}}\left(\tau_{1}\right)= \\
& =\frac{\eta V}{4 i \hbar} \int \frac{\mathrm{d}^{d} k}{(2 \pi)^{d}} \int_{0}^{\hbar \beta} d \tau \sum_{n m} \sum_{n^{\prime} m^{\prime}} \sum_{n^{\prime \prime} m^{\prime \prime}}[ \\
& -\mathfrak{B}_{\boldsymbol{k n m}}^{(2)} \mathfrak{A}_{\boldsymbol{k n n ^ { \prime } \boldsymbol { m } ^ { \prime }}}^{(1)} \mathfrak{C}_{\boldsymbol{k}_{-} n^{\prime \prime} m^{\prime \prime}} \mathscr{Z}_{\boldsymbol{k n m} n^{\prime} m^{\prime} n^{\prime \prime} m^{\prime \prime}}^{(3)}\left(\tau, \tau_{1}\right) \\
& -\mathfrak{B}_{\boldsymbol{k n m}}^{(2)} \mathfrak{A}_{\boldsymbol{k} n^{\prime} \boldsymbol{m}^{\prime}}^{(1)} \mathfrak{C}_{\boldsymbol{k}_{+} n^{\prime \prime} m^{\prime \prime}} \mathscr{Z}_{\boldsymbol{k n m} n^{\prime} m^{\prime} n^{\prime \prime} m^{\prime \prime}}^{(4)}\left(\tau, \tau_{1}\right) \\
& +\mathfrak{B}_{\boldsymbol{k n m}}^{(1)} \mathfrak{A}_{\boldsymbol{k} n^{\prime} \boldsymbol{m}^{\prime}}^{(2)} \mathfrak{C}_{\boldsymbol{k}_{+} n^{\prime \prime} m^{\prime \prime}} \mathscr{Z}_{\boldsymbol{k} n m n^{\prime} m^{\prime} n^{\prime \prime} m^{\prime \prime}}^{(5)}\left(\tau, \tau_{1}\right) \\
& \left.+\mathfrak{B}_{\boldsymbol{k n} n}^{(1)} \mathfrak{A}_{\boldsymbol{k} n^{\prime} m^{\prime}}^{(2)} \mathfrak{C}_{\boldsymbol{k}_{-} n^{\prime \prime} m^{\prime \prime}} \mathscr{Z}_{\boldsymbol{k n} n n^{\prime} m^{\prime} n^{\prime \prime} m^{\prime \prime}}^{(6)}\left(\tau, \tau_{1}\right)\right]
\end{aligned}
$$

within first-order perturbation theory, where we defined $\mathfrak{C}_{\boldsymbol{k}_{-} n^{\prime \prime} m^{\prime \prime}}=\left\langle u_{\boldsymbol{k}_{-} n^{\prime \prime}}|\mathfrak{C}| u_{\boldsymbol{k}_{-} m^{\prime \prime}}\right\rangle$ and $\mathfrak{C}_{\boldsymbol{k}_{+} n^{\prime \prime} m^{\prime \prime}}=$ $\left\langle u_{\boldsymbol{k}_{+} n^{\prime \prime}}|\mathfrak{C}| u_{\boldsymbol{k}_{+} m^{\prime \prime}}\right\rangle$.

Note that $\mathscr{Z}^{(5)}$ can be obtained from $\mathscr{Z}^{(3)}$ by replacing $\boldsymbol{k}_{-}$by $\boldsymbol{k}_{+}$and $\boldsymbol{k}_{+}$by $\boldsymbol{k}_{-}$. Similarly, $\mathscr{Z}^{(6)}$ can be obtained from $\mathscr{Z}^{(4)}$ by replacing $\boldsymbol{k}_{-}$by $\boldsymbol{k}_{+}$and $\boldsymbol{k}_{+}$by $\boldsymbol{k}_{-}$. Therefore, we write down only the equations for
$\mathscr{Z}^{(3)}$ and $\mathscr{Z}^{(4)}$ in the following. Using Wick's theorem we find

$$
\begin{aligned}
& \mathscr{Z}_{\boldsymbol{k} n m n^{\prime} m^{\prime} n^{\prime \prime} m^{\prime \prime}}^{(3)}\left(\tau, \tau_{1}\right)= \\
=- & G_{m^{\prime} n}^{\mathrm{M}}\left(\boldsymbol{k}_{-}, \tau_{1}-\tau\right) G_{m n^{\prime}}^{\mathrm{M}}\left(\boldsymbol{k}_{+}, \tau-\tau_{1}\right) G_{m^{\prime \prime} n^{\prime \prime}}^{\mathrm{M}}\left(\boldsymbol{k}_{-}, 0\right) \\
+ & G_{m n^{\prime}}^{\mathrm{M}}\left(\boldsymbol{k}_{+}, \tau-\tau_{1}\right) G_{m^{\prime \prime} n}^{\mathrm{M}}\left(\boldsymbol{k}_{-},-\tau\right) G_{m^{\prime} n^{\prime \prime}}^{\mathrm{M}}\left(\boldsymbol{k}_{-}, \tau_{1}\right)
\end{aligned}
$$

and

$$
\begin{aligned}
& \mathscr{Z}_{\boldsymbol{k} n m n^{\prime} m^{\prime} n^{\prime \prime} m^{\prime \prime}}^{(4)}\left(\tau, \tau_{1}\right)= \\
& =-G_{m n^{\prime}}^{\mathrm{M}}\left(\boldsymbol{k}_{+}, \tau-\tau_{1}\right) G_{m^{\prime} n}^{\mathrm{M}}\left(\boldsymbol{k}_{-}, \tau_{1}-\tau\right) G_{m^{\prime \prime} n^{\prime \prime}}^{\mathrm{M}}\left(\boldsymbol{k}_{+}, 0\right) \\
& +G_{m n^{\prime \prime}}^{\mathrm{M}}\left(\boldsymbol{k}_{+}, \tau\right) G_{m^{\prime} n}^{\mathrm{M}}\left(\boldsymbol{k}_{-}, \tau_{1}-\tau\right) G_{m^{\prime \prime} n^{\prime}}^{\mathrm{M}}\left(\boldsymbol{k}_{+},-\tau_{1}\right) \text {. }
\end{aligned}
$$

The Fourier transform

$$
\begin{aligned}
& \langle\langle\cos (\boldsymbol{q} \cdot \boldsymbol{r}) \mathfrak{A} ; \mathfrak{C}\rangle\rangle^{\mathrm{M}}\left(i \mathcal{E}_{N}\right)= \\
= & \int_{0}^{\hbar \beta} d \tau_{1} e^{\frac{i}{\hbar} \mathcal{E}_{N} \tau_{1}}\langle\langle\cos (\boldsymbol{q} \cdot \boldsymbol{r}) \mathfrak{A} ; \mathfrak{C}\rangle\rangle^{\mathrm{M}}\left(\tau_{1}\right)
\end{aligned}
$$

of Eq. (B10) can be written as

$$
\begin{aligned}
& \langle\langle\cos (\boldsymbol{q} \cdot \boldsymbol{r}) \mathfrak{A} ; \mathfrak{C}\rangle\rangle^{\mathrm{M}}\left(i \mathcal{E}_{N}\right)= \\
& =\frac{\eta V}{4 i \hbar} \int \frac{\mathrm{d}^{d} k}{(2 \pi)^{d}} \sum_{n m} \sum_{n^{\prime} m^{\prime}} \sum_{n^{\prime \prime} m^{\prime \prime}}[ \\
& -\mathfrak{B}_{\boldsymbol{k n m}}^{(2)} \mathfrak{A}_{\boldsymbol{k} n^{\prime} m^{\prime}}^{(1)} \mathfrak{C}_{\boldsymbol{k}_{-} n^{\prime \prime} m^{\prime \prime}} \mathscr{Z}_{\boldsymbol{k n m} n n^{\prime} m^{\prime} n^{\prime \prime} m^{\prime \prime}}^{(3 a)}\left(i \mathcal{E}_{N}\right) \\
& -\mathfrak{B}_{\boldsymbol{k n m}}^{(2)} \mathfrak{A}_{\boldsymbol{k} n^{\prime} m^{\prime}}^{(1)} \mathfrak{C}_{\boldsymbol{k}_{+} n^{\prime \prime} m^{\prime \prime}} \mathscr{Z}_{\boldsymbol{k} n m n^{\prime} m^{\prime} n^{\prime \prime} m^{\prime \prime}}^{(4 a)}\left(i \mathcal{E}_{N}\right) \\
& +\mathfrak{B}_{\boldsymbol{k n m}}^{(1)} \mathfrak{A}_{\boldsymbol{k n} n^{\prime} m^{\prime}}^{(2)} \mathfrak{C}_{\boldsymbol{k}_{+} n^{\prime \prime} m^{\prime \prime}} \mathscr{Z}_{\boldsymbol{k} n m n^{\prime} m^{\prime} n^{\prime \prime} m^{\prime \prime}}^{(5 a)}\left(i \mathcal{E}_{N}\right) \\
& \left.+\mathfrak{B}_{\boldsymbol{k n m}}^{(1)} \mathfrak{A}_{\boldsymbol{k} n^{\prime} \boldsymbol{m}^{\prime}}^{(2)} \mathfrak{C}_{\boldsymbol{k}_{-} n^{\prime \prime} \boldsymbol{m}^{\prime \prime}} \mathscr{Z}_{\boldsymbol{k} n m n^{\prime} \boldsymbol{m}^{\prime} \boldsymbol{n}^{\prime \prime} \boldsymbol{m}^{\prime \prime}}^{(6 a)}\left(i \mathcal{E}_{N}\right)\right]
\end{aligned}
$$

in terms of the integrals

$$
\begin{aligned}
& \mathscr{Z}_{\boldsymbol{k} n m n^{\prime} m^{\prime} n^{\prime \prime} m^{\prime \prime}}^{(3 a)}\left(i \mathcal{E}_{N}\right)=\int_{0}^{\hbar \beta} d \tau \int_{0}^{\hbar \beta} d \tau_{1} e^{\frac{i}{\hbar} \mathcal{E}_{N} \tau_{1}} \times \\
& \times G_{m n^{\prime}}^{\mathrm{M}}\left(\boldsymbol{k}_{+}, \tau-\tau_{1}\right) G_{m^{\prime \prime} n}^{\mathrm{M}}\left(\boldsymbol{k}_{-},-\tau\right) G_{m^{\prime} n^{\prime \prime}}^{\mathrm{M}}\left(\boldsymbol{k}_{-}, \tau_{1}\right)= \\
& =\frac{1}{\hbar \beta} \sum_{p} G_{\boldsymbol{k}_{+} m n^{\prime}}^{\mathrm{M}}\left(i \mathcal{E}_{p}\right) G_{\boldsymbol{k}_{-} m^{\prime \prime} n}^{\mathrm{M}}\left(i \mathcal{E}_{p}\right) G_{\boldsymbol{k}_{-} m^{\prime} n^{\prime \prime}}^{\mathrm{M}}\left(i \mathcal{E}_{p}+i \mathcal{E}_{N}\right)
\end{aligned}
$$

and

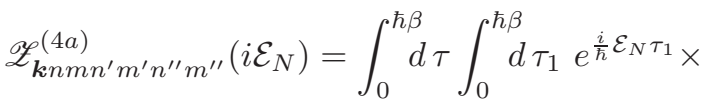

$$
\begin{aligned}
& \times G_{m n^{\prime \prime}}^{\mathrm{M}}\left(\boldsymbol{k}_{+}, \tau\right) G_{m^{\prime} n}^{\mathrm{M}}\left(\boldsymbol{k}_{-}, \tau_{1}-\tau\right) G_{m^{\prime \prime} n^{\prime}}^{\mathrm{M}}\left(\boldsymbol{k}_{+},-\tau_{1}\right)= \\
& =\frac{1}{\hbar \beta} \sum_{p} G_{\boldsymbol{k}_{+} m n^{\prime \prime}}^{\mathrm{M}}\left(i \mathcal{E}_{p}\right) G_{\boldsymbol{k}_{-} m^{\prime} n}^{\mathrm{M}}\left(i \mathcal{E}_{p}\right) G_{\boldsymbol{k}_{+} m^{\prime \prime} n^{\prime}}^{\mathrm{M}}\left(i \mathcal{E}_{p}-i \mathcal{E}_{N}\right) \text {, }
\end{aligned}
$$

where $\mathcal{E}_{N}=2 \pi N / \beta$ is a bosonic Matsubara energy point and we used

$$
G^{\mathrm{M}}(\tau)=\frac{1}{\hbar \beta} \sum_{p=-\infty}^{\infty} e^{-i \mathcal{E}_{p} \tau / \hbar} G^{\mathrm{M}}\left(i \mathcal{E}_{p}\right),
$$


where $\mathcal{E}_{p}=(2 p+1) \pi / \beta$ is a fermionic Matsubara point. Again $\mathscr{Z}^{(5 a)}$ is obtained from $\mathscr{Z}^{(3 a)}$ by replacing $\boldsymbol{k}_{-}$by $\boldsymbol{k}_{+}$and $\boldsymbol{k}_{+}$by $\boldsymbol{k}_{-}$and $\mathscr{Z}^{(6 a)}$ is obtained from $\mathscr{Z}^{(4 a)}$ in the same way.

Summation over Matsubara points $\mathcal{E}_{p}$ in Eq. (B15) and in Eq. (B16) and analytic continuation $i \mathcal{E}_{N} \rightarrow \hbar \omega$ yields

$$
\begin{aligned}
& 2 \pi i \hbar \mathscr{Z}_{\boldsymbol{k n m} n^{\prime} m^{\prime} n^{\prime \prime} m^{\prime \prime}}^{(3 a)}(\hbar \omega)= \\
& -\int d \mathcal{E} f(\mathcal{E}) G_{\boldsymbol{k}_{+} m n^{\prime}}^{\mathrm{R}}(\mathcal{E}) G_{\boldsymbol{k}_{-} m^{\prime \prime} n}^{\mathrm{R}}(\mathcal{E}) G_{\boldsymbol{k}_{-} m^{\prime} n^{\prime \prime}}^{\mathrm{R}}(\mathcal{E}+\hbar \omega) \\
& +\int d \mathcal{E} f(\mathcal{E}) G_{\boldsymbol{k}_{+} m n^{\prime}}^{\mathrm{A}}(\mathcal{E}) G_{\boldsymbol{k}_{-} m^{\prime \prime} n}^{\mathrm{A}}(\mathcal{E}) G_{\boldsymbol{k}_{-} m^{\prime} n^{\prime \prime}}^{\mathrm{R}}(\mathcal{E}+\hbar \omega) \\
& -\int d \mathcal{E} f(\mathcal{E}) G_{\boldsymbol{k}_{+} m n^{\prime}}^{\mathrm{A}}(\mathcal{E}-\hbar \omega) G_{\boldsymbol{k}_{-} m^{\prime \prime} n}^{\mathrm{A}}(\mathcal{E}-\hbar \omega) G_{\boldsymbol{k}_{-} m^{\prime} n^{\prime \prime}}^{\mathrm{R}}(\mathcal{E}) \\
& +\int d \mathcal{E} f(\mathcal{E}) G_{\boldsymbol{k}_{+} m n^{\prime}}^{\mathrm{A}}(\mathcal{E}-\hbar \omega) G_{\boldsymbol{k}_{-} m^{\prime \prime} n}^{\mathrm{A}}(\mathcal{E}-\hbar \omega) G_{\boldsymbol{k}_{-} m^{\prime} n^{\prime \prime}}^{\mathrm{A}}(\mathcal{E})
\end{aligned}
$$

and

$$
\begin{aligned}
& 2 \pi i \hbar \mathscr{Z}_{\boldsymbol{k} n m n^{\prime} m^{\prime} n^{\prime \prime} m^{\prime \prime}}^{(4 a)}(\hbar \omega)= \\
& -\int d \mathcal{E} f(\mathcal{E}) G_{\boldsymbol{k}_{+} m n^{\prime \prime}}^{\mathrm{R}}(\mathcal{E}) G_{\boldsymbol{k}_{-} m^{\prime} n}^{\mathrm{R}}(\mathcal{E}) G_{\boldsymbol{k}_{+} m^{\prime \prime} n^{\prime}}^{\mathrm{A}}(\mathcal{E}-\hbar \omega) \\
& +\int d \mathcal{E} f(\mathcal{E}) G_{\boldsymbol{k}_{+} m n^{\prime \prime}}^{\mathrm{A}}(\mathcal{E}) G_{\boldsymbol{k}_{-} m^{\prime} n}^{\mathrm{A}}(\mathcal{E}) G_{\boldsymbol{k}_{+} m^{\prime \prime} n^{\prime}}^{\mathrm{A}}(\mathcal{E}-\hbar \omega) \\
& -\int d \mathcal{E} f(\mathcal{E}) G_{\boldsymbol{k}_{+} m n^{\prime \prime}}^{\mathrm{R}}(\mathcal{E}+\hbar \omega) G_{\boldsymbol{k}_{-} m^{\prime} n}^{\mathrm{R}}(\mathcal{E}+\hbar \omega) G_{\boldsymbol{k}_{+} m^{\prime \prime} n^{\prime}}^{\mathrm{R}}(\mathcal{E}) \\
& +\int d \mathcal{E} f(\mathcal{E}) G_{\boldsymbol{k}_{+} m n^{\prime \prime}}^{\mathrm{R}}(\mathcal{E}+\hbar \omega) G_{\boldsymbol{k}_{-} m^{\prime} n}^{\mathrm{R}}(\mathcal{E}+\hbar \omega) G_{\boldsymbol{k}_{+} m^{\prime \prime} n^{\prime}}^{\mathrm{A}}(\mathcal{E}) .
\end{aligned}
$$

In the next step we take the limit $\omega \rightarrow 0$ (see Eq. (64), Eq. (70), and Eq. (77)):

$$
\begin{aligned}
& -\frac{1}{V} \lim _{\omega \rightarrow 0} \frac{\operatorname{Im}\langle\langle\mathcal{A} \cos (\boldsymbol{q} \cdot \boldsymbol{r}) ; \mathfrak{C}\rangle\rangle^{\mathrm{R}}(\hbar \omega)}{\hbar \omega}= \\
& =\frac{\eta}{4 \hbar} \operatorname{Im}\left[\mathscr{Y}^{(3)}+\mathscr{Y}^{(4)}-\mathscr{Y}^{(5)}-\mathscr{Y}^{(6)}\right],
\end{aligned}
$$

where we defined

$$
\begin{aligned}
& \mathscr{Y}^{(3)}=\frac{1}{i \hbar} \int \frac{\mathrm{d}^{d} k}{(2 \pi)^{d}} \sum_{n m} \sum_{n^{\prime} m^{\prime}} \sum_{n^{\prime \prime} m^{\prime \prime}} \mathfrak{B}_{\boldsymbol{k} n m}^{(2)} \mathfrak{A}_{\boldsymbol{k} n^{\prime} m^{\prime}}^{(1)} \mathfrak{C}_{\boldsymbol{k}_{-} n^{\prime \prime} m^{\prime \prime}} \times \\
& \times\left.\frac{\partial \mathscr{Z}_{\boldsymbol{k} n m n^{\prime} m^{\prime} n^{\prime \prime} m^{\prime \prime}}^{(3 a)}(\hbar \omega)}{\partial \omega}\right|_{\omega=0}, \\
& \mathscr{Y}^{(4)}=\frac{1}{i \hbar} \int \frac{\mathrm{d}^{d} k}{(2 \pi)^{d}} \sum_{n m} \sum_{n^{\prime} m^{\prime}} \sum_{n^{\prime \prime} m^{\prime \prime}} \mathfrak{B}_{\boldsymbol{k} n}^{(2)} \mathfrak{A}_{\boldsymbol{k} n^{\prime} m^{\prime}}^{(1)} \mathfrak{C}_{\boldsymbol{k}_{+} n^{\prime \prime} m^{\prime \prime}} \times
\end{aligned}
$$

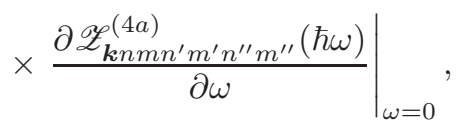

$$
\begin{aligned}
& \mathscr{Y}^{(5)}=\frac{1}{i \hbar} \int \frac{\mathrm{d}^{d} k}{(2 \pi)^{d}} \sum_{n m} \sum_{n^{\prime} m^{\prime}} \sum_{n^{\prime \prime} m^{\prime \prime}} \mathfrak{B}_{\boldsymbol{k} n}^{(1)} \mathfrak{A}_{\boldsymbol{k} n^{\prime} m^{\prime}}^{(2)} \mathfrak{C}_{\boldsymbol{k}_{+} n^{\prime \prime} m^{\prime \prime}} \times \\
& \times\left.\frac{\partial \mathscr{Z}_{k n m n^{\prime} m^{\prime} n^{\prime \prime} m^{\prime \prime}}^{(5 a)}}{\partial \omega}\right|_{\omega=0}, \\
& \mathscr{Y}^{(6)}=\frac{1}{i \hbar} \int \frac{\mathrm{d}^{d} k}{(2 \pi)^{d}} \sum_{n m} \sum_{n^{\prime} m^{\prime}} \sum_{n^{\prime \prime} m^{\prime \prime}} \mathfrak{B}_{\boldsymbol{k} n m^{(1)}}^{(1)} \mathfrak{A}_{\boldsymbol{k} n^{\prime} m^{\prime}}^{(2)} \mathfrak{C}_{\boldsymbol{k}_{-} n^{\prime \prime} m^{\prime \prime}} \times \\
& \times\left.\frac{\partial \mathscr{Z}_{\boldsymbol{k n m} n^{\prime} m^{\prime} n^{\prime \prime} m^{\prime \prime}}^{(6 a)}}{\partial \omega}\right|_{\omega=0},
\end{aligned}
$$

which can be expressed as $\mathscr{Y}^{(3)}=\mathscr{Y}^{(3 a)}+\mathscr{Y}^{(3 b)}$ and $\mathscr{Y}^{(4)}=\mathscr{Y}^{(4 a)}+\mathscr{Y}^{(4 b)}$, where

$$
\begin{aligned}
2 \pi \hbar \mathscr{Y}^{(3 a)} & =\frac{1}{\hbar} \int \frac{\mathrm{d}^{d} k}{(2 \pi)^{d}} \int d \mathcal{E} f(\mathcal{E}) \times \\
\times \operatorname{Tr}[ & \mathfrak{A}_{\boldsymbol{k}} G_{\boldsymbol{k}_{-}}^{\mathrm{R}}(\mathcal{E}) \mathfrak{C}_{\boldsymbol{k}_{-}} G_{\boldsymbol{k}_{-}}^{\mathrm{A}}(\mathcal{E}) \mathfrak{B}_{\boldsymbol{k}} G_{\boldsymbol{k}_{+}}^{\mathrm{A}}(\mathcal{E}) G_{\boldsymbol{k}_{+}}^{\mathrm{A}}(\mathcal{E}) \\
+ & \mathfrak{A}_{\boldsymbol{k}} G_{\boldsymbol{k}_{-}}^{\mathrm{R}}(\mathcal{E}) G_{\boldsymbol{k}_{-}}^{\mathrm{R}}(\mathcal{E}) \mathfrak{C}_{\boldsymbol{k}_{-}} G_{\boldsymbol{k}_{-}}^{\mathrm{A}}(\mathcal{E}) \mathfrak{B}_{k} G_{\boldsymbol{k}_{+}}^{\mathrm{A}}(\mathcal{E}) \\
+ & \left.\mathfrak{A}_{\boldsymbol{k}} G_{\boldsymbol{k}_{-}}^{\mathrm{R}}(\mathcal{E}) \mathfrak{C}_{\boldsymbol{k}_{-}} G_{\boldsymbol{k}_{-}}^{\mathrm{A}}(\mathcal{E}) G_{\boldsymbol{k}_{-}}^{\mathrm{A}}(\mathcal{E}) \mathfrak{B}_{\boldsymbol{k}} G_{\boldsymbol{k}_{+}}^{\mathrm{A}}(\mathcal{E})\right] \\
= & \int \frac{\mathrm{d}^{d} k}{(2 \pi)^{d}} \int d \mathcal{E} f^{\prime}(\mathcal{E}) \times \\
& \times \operatorname{Tr}\left[\mathfrak{A}_{\boldsymbol{k}} G_{\boldsymbol{k}_{-}}^{\mathrm{R}}(\mathcal{E}) \mathfrak{C}_{\boldsymbol{k}_{-}} G_{\boldsymbol{k}_{-}}^{\mathrm{A}}(\mathcal{E}) \mathfrak{B}_{\boldsymbol{k}} G_{\boldsymbol{k}_{+}}^{\mathrm{A}}(\mathcal{E})\right]
\end{aligned}
$$

and

$$
\begin{gathered}
2 \pi \hbar \mathscr{Y}^{(3 b)}=-\frac{1}{\hbar} \int \frac{\mathrm{d}^{d} k}{(2 \pi)^{d}} \int d \mathcal{E} f(\mathcal{E}) \times \\
\times \operatorname{Tr}\left[\mathfrak{A}_{\boldsymbol{k}} G_{\boldsymbol{k}_{-}}^{\mathrm{A}}(\mathcal{E}) \mathfrak{C}_{\boldsymbol{k}_{-}} G_{\boldsymbol{k}_{-}}^{\mathrm{A}}(\mathcal{E}) \mathfrak{B}_{\boldsymbol{k}} G_{\boldsymbol{k}_{+}}^{\mathrm{A}}(\mathcal{E}) G_{\boldsymbol{k}_{+}}^{\mathrm{A}}(\mathcal{E})\right. \\
+\mathfrak{A}_{\boldsymbol{k}} G_{\boldsymbol{k}_{-}}^{\mathrm{R}}(\mathcal{E}) G_{\boldsymbol{k}_{-}}^{\mathrm{R}}(\mathcal{E}) \mathfrak{C}_{\boldsymbol{k}_{-}} G_{\boldsymbol{k}_{-}}^{\mathrm{R}}(\mathcal{E}) \mathfrak{B}_{\boldsymbol{k}} G_{\boldsymbol{k}_{+}}^{\mathrm{R}}(\mathcal{E}) \\
\left.+\mathfrak{A}_{\boldsymbol{k}} G_{\boldsymbol{k}_{-}}^{\mathrm{A}}(\mathcal{E}) \mathfrak{C}_{\boldsymbol{k}_{-}} G_{\boldsymbol{k}_{-}}^{\mathrm{A}}(\mathcal{E}) G_{\boldsymbol{k}_{-}}^{\mathrm{A}}(\mathcal{E}) \mathfrak{B}_{\boldsymbol{k}} G_{\boldsymbol{k}_{+}}^{\mathrm{A}}(\mathcal{E})\right] .
\end{gathered}
$$


Similarly,

$$
\begin{aligned}
& 2 \pi \hbar \mathscr{Y}^{(4 a)}= \frac{1}{\hbar} \int \frac{\mathrm{d}^{d} k}{(2 \pi)^{d}} \int d \mathcal{E} f(\mathcal{E}) \times \\
& \times \operatorname{Tr}\left[\mathfrak{A}_{\boldsymbol{k}} G_{\boldsymbol{k}_{-}}^{\mathrm{R}}(\mathcal{E}) \mathfrak{B}_{\boldsymbol{k}} G_{\boldsymbol{k}_{+}}^{\mathrm{R}}(\mathcal{E}) \mathfrak{C}_{\boldsymbol{k}_{+}} G_{\boldsymbol{k}_{+}}^{\mathrm{A}}(\mathcal{E}) G_{\boldsymbol{k}_{+}}^{\mathrm{A}}(\mathcal{E})\right. \\
& \quad-\mathfrak{A}_{\boldsymbol{k}} G_{\boldsymbol{k}_{-}}^{\mathrm{R}}(\mathcal{E}) G_{\boldsymbol{k}_{-}}^{\mathrm{R}}(\mathcal{E}) \mathfrak{B}_{k} G_{\boldsymbol{k}_{+}}^{\mathrm{R}}(\mathcal{E}) \mathfrak{C}_{\boldsymbol{k}_{+}} G_{\boldsymbol{k}_{+}}^{\mathrm{A}}(\mathcal{E}) \\
&\left.-\mathfrak{A}_{\boldsymbol{k}} G_{\boldsymbol{k}_{-}}^{\mathrm{R}}(\mathcal{E}) \mathfrak{B}_{\boldsymbol{k}} G_{\boldsymbol{k}_{+}}^{\mathrm{R}}(\mathcal{E}) G_{\boldsymbol{k}_{+}}^{\mathrm{R}}(\mathcal{E}) \mathfrak{C}_{\boldsymbol{k}_{+}} G_{\boldsymbol{k}_{+}}^{\mathrm{A}}(\mathcal{E})\right] \\
&=\int \frac{\mathrm{d}^{d} k}{(2 \pi)^{d}} \int d \mathcal{E} f^{\prime}(\mathcal{E}) \times \\
& \quad \times \operatorname{Tr}\left[\mathfrak{A}_{\boldsymbol{k}} G_{\boldsymbol{k}_{-}}^{\mathrm{R}}(\mathcal{E}) \mathfrak{B}_{\boldsymbol{k}} G_{\boldsymbol{k}_{+}}^{\mathrm{R}}(\mathcal{E}) \mathfrak{C}_{\boldsymbol{k}_{+}} G_{\boldsymbol{k}_{+}}^{\mathrm{A}}(\mathcal{E})\right]
\end{aligned}
$$

and

$$
\begin{gathered}
2 \pi \hbar \mathscr{Y}^{(4 b)}=-\frac{1}{\hbar} \int \frac{\mathrm{d}^{d} k}{(2 \pi)^{d}} \int d \mathcal{E} f(\mathcal{E}) \times \\
\times \operatorname{Tr}\left[\mathfrak{A}_{\boldsymbol{k}} G_{\boldsymbol{k}_{-}}^{\mathrm{A}}(\mathcal{E}) \mathfrak{B}_{k} G_{\boldsymbol{k}_{+}}^{\mathrm{A}}(\mathcal{E}) \mathfrak{C}_{\boldsymbol{k}_{+}} G_{\boldsymbol{k}_{+}}^{\mathrm{A}}(\mathcal{E}) G_{\boldsymbol{k}_{+}}^{\mathrm{A}}(\mathcal{E})\right. \\
+\mathfrak{A}_{\boldsymbol{k}} G_{\boldsymbol{k}_{-}}^{\mathrm{R}}(\mathcal{E}) G_{\boldsymbol{k}_{-}}^{\mathrm{R}}(\mathcal{E}) \mathfrak{B}_{\boldsymbol{k}} G_{\boldsymbol{k}_{+}}^{\mathrm{R}}(\mathcal{E}) \mathfrak{C}_{\boldsymbol{k}_{+}} G_{\boldsymbol{k}_{+}}^{\mathrm{R}}(\mathcal{E}) \\
\left.+\mathfrak{A}_{\boldsymbol{k}} G_{\boldsymbol{k}_{-}}^{\mathrm{R}}(\mathcal{E}) \mathfrak{B}_{\boldsymbol{k}} G_{\boldsymbol{k}_{+}}^{\mathrm{R}}(\mathcal{E}) G_{\boldsymbol{k}_{+}}^{\mathrm{R}}(\mathcal{E}) \mathfrak{C}_{\boldsymbol{k}_{+}} G_{\boldsymbol{k}_{+}}^{\mathrm{R}}(\mathcal{E})\right] .
\end{gathered}
$$

We call $\mathscr{Y}^{(3 a)}$ and $\mathscr{Y}^{(4 a)}$ Fermi surface terms and $\mathscr{Y}^{(3 b)}$ and $\mathscr{Y}^{(4 b)}$ Fermi sea terms. Again $\mathscr{Y}^{(5)}$ is obtained from $\mathscr{Y}^{(3)}$ by replacing $\boldsymbol{k}_{-}$by $\boldsymbol{k}_{+}$and $\boldsymbol{k}_{+}$by $\boldsymbol{k}_{-}$and $\mathscr{Y}^{(6)}$ is obtained from $\mathscr{Y}^{(4)}$ in the same way.

Finally, we take the limit $\boldsymbol{q} \rightarrow 0$ :

$$
\begin{aligned}
& \Lambda=-\frac{2}{\hbar V \eta} \operatorname{Im} \lim _{\boldsymbol{q} \rightarrow 0} \lim _{\omega \rightarrow 0} \frac{\partial}{\partial \omega} \frac{\partial}{\partial q_{i}}\langle\langle\mathcal{A} \cos (\boldsymbol{q} \cdot \boldsymbol{r}) ; \mathfrak{C}\rangle\rangle^{\mathrm{R}}(\hbar \omega) \\
& =\frac{1}{2 \hbar} \lim _{\boldsymbol{q} \rightarrow 0} \frac{\partial}{\partial q_{i}} \operatorname{Im}\left[\mathscr{Y}^{(3)}+\mathscr{Y}^{(4)}-\mathscr{Y}^{(5)}-\mathscr{Y}^{(6)}\right] \\
& =\frac{1}{2 \hbar} \operatorname{Im}\left[\mathscr{X}^{(3)}+\mathscr{X}^{(4)}-\mathscr{X}^{(5)}-\mathscr{X}^{(6)}\right]
\end{aligned}
$$

where we defined

$$
\mathscr{X}^{(j)}=\left.\frac{\partial}{\partial q_{i}}\right|_{\boldsymbol{q}=0} \mathscr{Y}^{(j)}
$$

for $j=3,4,5,6$. Since $\mathscr{Y}^{(4)}$ and $\mathscr{Y}^{(6)}$ are related by the interchange of $\boldsymbol{k}_{-}$and $\boldsymbol{k}_{+}$it follows that $\mathscr{X}^{(6)}=$ $-\mathscr{X}^{(4)}$. Similarly, since $\mathscr{Y}^{(3)}$ and $\mathscr{Y}^{(5)}$ are related by the interchange of $\boldsymbol{k}_{-}$and $\boldsymbol{k}_{+}$it follows that $\mathscr{X}^{(5)}=-\mathscr{X}^{(3)}$. Consequently, we need

$$
\Lambda=\frac{1}{\hbar} \operatorname{Im}\left[\mathscr{X}^{(3 a)}+\mathscr{X}^{(3 b)}+\mathscr{X}^{(4 a)}+\mathscr{X}^{(4 b)}\right],
$$

where $\mathscr{X}^{(3 a)}$ and $\mathscr{X}^{(4 a)}$ are the Fermi surface terms and $\mathscr{X}^{(3 b)}$ and $\mathscr{X}^{(4 b)}$ are the Fermi sea terms. The Fermi surface terms are given by

$$
\begin{array}{r}
\mathscr{X}^{(3 a)}=\frac{-1}{4 \pi \hbar} \int \frac{\mathrm{d}^{d} k}{(2 \pi)^{d}} \int d \mathcal{E} f^{\prime}(\mathcal{E}) \operatorname{Tr}[ \\
\mathfrak{A}_{k} G_{\boldsymbol{k}}^{\mathrm{R}}(\mathcal{E}) v_{\boldsymbol{k}} G_{\boldsymbol{k}}^{\mathrm{R}}(\mathcal{E}) \mathfrak{C}_{k} G_{\boldsymbol{k}}^{\mathrm{A}}(\mathcal{E}) \mathfrak{B}_{k} G_{\boldsymbol{k}}^{\mathrm{A}}(\mathcal{E}) \\
+\mathfrak{A}_{\boldsymbol{k}} G_{\boldsymbol{k}}^{\mathrm{R}}(\mathcal{E}) \mathfrak{C}_{\boldsymbol{k}} G_{\boldsymbol{k}}^{\mathrm{A}}(\mathcal{E}) v_{\boldsymbol{k}} G_{\boldsymbol{k}}^{\mathrm{A}}(\mathcal{E}) \mathfrak{B}_{k} G_{\boldsymbol{k}}^{\mathrm{A}}(\mathcal{E}) \\
-\mathfrak{A}_{\boldsymbol{k}} G_{\boldsymbol{k}}^{\mathrm{R}}(\mathcal{E}) \mathfrak{C}_{\boldsymbol{k}} G_{\boldsymbol{k}}^{\mathrm{A}}(\mathcal{E}) \mathfrak{B}_{\boldsymbol{k}} G_{\boldsymbol{k}}^{\mathrm{A}}(\mathcal{E}) v_{\boldsymbol{k}} G_{\boldsymbol{k}}^{\mathrm{A}}(\mathcal{E}) \\
\left.+\mathfrak{A}_{\boldsymbol{k}} G_{\boldsymbol{k}}^{\mathrm{R}}(\mathcal{E}) \frac{\partial \mathfrak{C}_{\boldsymbol{k}}}{\partial k} G_{\boldsymbol{k}}^{\mathrm{A}}(\mathcal{E}) \mathfrak{B}_{\boldsymbol{k}} G_{\boldsymbol{k}}^{\mathrm{A}}(\mathcal{E})\right]
\end{array}
$$

and

$$
\mathscr{X}^{(4 a)}=-\left[\mathscr{X}^{(3 a)}\right]^{*} .
$$

The Fermi sea terms are given by

$$
\begin{aligned}
\mathscr{X}^{(3 b)} & =\frac{-1}{4 \pi \hbar^{2}} \int \frac{\mathrm{d}^{d} k}{(2 \pi)^{d}} \int d \mathcal{E} f(\mathcal{E}) \operatorname{Tr}[ \\
& -(\mathfrak{A} R v R R \mathfrak{C} R \mathfrak{B} R)+(\mathfrak{A} A \mathfrak{C} A A \mathfrak{B} A v A) \\
& -(\mathfrak{A} R R v R \mathfrak{C} R \mathfrak{B} R)-(\mathfrak{A} R R \mathfrak{C} R v R \mathfrak{B} R) \\
& +(\mathfrak{A} R R \mathfrak{C} R \mathfrak{B} R v R)-(\mathfrak{A} A v A \mathfrak{C} A \mathfrak{B} A A) \\
& -(\mathfrak{A} A \mathfrak{C} A v A \mathfrak{B} A A)+(\mathfrak{A} A \mathfrak{C} A \mathfrak{B} A v A A) \\
& +(\mathfrak{A} A \mathfrak{C} A \mathfrak{B} A A v A)-(\mathfrak{A} A v A \mathfrak{C} A A \mathfrak{B} A) \\
& -(\mathfrak{A} A \mathfrak{C} A v A A \mathfrak{B} A)-(\mathfrak{A} A \mathfrak{C} A A v A \mathfrak{B} A) \\
& -\left(\mathfrak{A} R R \frac{\partial \mathfrak{C}}{\partial k} R \mathfrak{B} R\right)-\left(\mathfrak{A} A \frac{\partial \mathfrak{C}}{\partial k} A A \mathfrak{B} A\right) \\
& \left.-\left(\mathfrak{A} A \frac{\partial \mathfrak{C}}{\partial k} A \mathfrak{B} A A\right)\right]
\end{aligned}
$$

and

$$
\mathscr{X}^{(4 b)}=-\left[\mathscr{X}^{(3 b)}\right]^{*} .
$$

In Eq. (B31) we use the abbreviations $R=G_{\boldsymbol{k}}^{\mathrm{R}}(\mathcal{E}), A=$ $G_{\boldsymbol{k}}^{\mathrm{A}}(\mathcal{E}), \mathfrak{A}=\mathfrak{A}_{\boldsymbol{k}}, \mathfrak{B}=\mathfrak{B}_{\boldsymbol{k}}, \mathfrak{C}=\mathfrak{C}_{\boldsymbol{k}}$. It is important to note that $\mathfrak{C}_{\boldsymbol{k}_{-}}$and $\mathfrak{C}_{\boldsymbol{k}_{+}}$depend on $q$ through $\boldsymbol{k}_{-}=$ $\boldsymbol{k}-\boldsymbol{q} / 2$ and $\boldsymbol{k}_{+}=\boldsymbol{k}+\boldsymbol{q} / 2$. The $q$ derivative therefore generates the additional terms with $\partial \mathfrak{C}_{k} / \partial k$ in Eq. (B29) and Eq. (B31). In contrast, $\mathfrak{A}_{\boldsymbol{k}}$ and $\mathfrak{B}_{\boldsymbol{k}}$ do not depend linearly on $q$.

Eq. (B28) simplifies due to the relations Eq. (B30) and Eq. (B32) as follows:

$$
\Lambda=\frac{2}{\hbar} \operatorname{Im}\left[\mathscr{X}^{(3 a)}+\mathscr{X}^{(3 b)}\right] .
$$

In order to obtain the expression for the chiral contribution to the torque-torque correlation we choose the operators as follows:

$$
\begin{aligned}
& \mathfrak{B} \rightarrow \mathcal{T}_{k} \\
& \mathfrak{A} \rightarrow-\mathcal{T}_{i} \\
& \mathfrak{C} \rightarrow \mathcal{T}_{j} \\
& \frac{\partial \mathfrak{C}}{\partial k}=0 \\
& v \rightarrow v_{l} .
\end{aligned}
$$


This leads to Eq. (78), Eq. (79) and Eq. (80) of the main text.

In order to obtain the expression for the chiral contribution to the CIT, we set

$$
\begin{aligned}
& \mathfrak{B} \rightarrow \mathcal{T}_{k} \\
& \mathfrak{A} \rightarrow-\mathcal{T}_{i} \\
& \mathfrak{C} \rightarrow-e v_{j} \\
& \frac{\partial \mathfrak{C}}{\partial k} \rightarrow-\frac{e \hbar}{m} \delta_{j l} \\
& v \rightarrow v_{l} .
\end{aligned}
$$

This leads to Eq. (66), Eq. (67) and Eq. (68).

In order to obtain the expression for the chiral contribution to the ICIT, we set

$$
\begin{aligned}
& \mathfrak{B} \rightarrow \mathcal{T}_{k} \\
& \mathfrak{A} \rightarrow-e v_{i} \\
& \mathfrak{C} \rightarrow \mathcal{T}_{j} \\
& \frac{\partial \mathfrak{C}}{\partial k} \rightarrow 0 \\
& v \rightarrow v_{l} .
\end{aligned}
$$

This leads to Eq. (71), Eq. (72) and Eq. (73).

* Corresp. author: f.freimuth@fz-juelich.de

[1] K. Nawaoka, S. Miwa, Y. Shiota, N. Mizuochi, and Y. Suzuki, Applied Physics Express 8, 063004 (2015).

[2] H. Yang, O. Boulle, V. Cros, A. Fert, and M. Chshiev, Scientific Reports 8, 12356 (2018).

[3] T. Srivastava, M. Schott, R. Juge, V. Křižáková, M. Belmeguenai, Y. Roussigné, A. Bernand-Mantel, L. Ranno, S. Pizzini, S.-M. Chérif, et al., Nano Letters 18, 4871 (2018).

[4] R. V. Mikhaylovskiy, E. Hendry, A. Secchi, J. H. Mentink, M. Eckstein, A. Wu, R. V. Pisarev, V. V. Kruglyak, M. I. Katsnelson, T. Rasing, et al., Nature Communications 6, 8190 (2015).

[5] F. Freimuth, S. Blügel, and Y. Mokrousov, Phys. Rev. B 98, 024419 (2018).

[6] F. Freimuth, S. Blügel, and Y. Mokrousov, Phys. Rev. B 96, 054403 (2017).

[7] T. Kikuchi, T. Koretsune, R. Arita, and G. Tatara, Phys. Rev. Lett. 116, 247201 (2016).

[8] G. V. Karnad, F. Freimuth, E. Martinez, R. Lo Conte, G. Gubbiotti, T. Schulz, S. Senz, B. Ocker, Y. Mokrousov, and M. Kläui, Phys. Rev. Lett. 121, 147203 (2018).

[9] N. Kato, M. Kawaguchi, Y.-C. Lau, T. Kikuchi, Y. Nakatani, and M. Hayashi, Phys. Rev. Lett. 122, 257205 (2019).

[10] F. Freimuth, S. Blügel, and Y. Mokrousov, Journal of physics: Condensed matter 26, 104202 (2014).

[11] F. Freimuth, R. Bamler, Y. Mokrousov, and A. Rosch, Phys. Rev. B 88, 214409 (2013).

[12] J. Shi, G. Vignale, D. Xiao, and Q. Niu, Phys. Rev. Lett. 99, 197202 (2007).
[13] T. Thonhauser, D. Ceresoli, D. Vanderbilt, and R. Resta, Phys. Rev. Lett. 95, 137205 (2005).

[14] D. Ceresoli, T. Thonhauser, D. Vanderbilt, and R. Resta, Phys. Rev. B 74, 024408 (2006).

[15] A. Malashevich, I. Souza, S. Coh, and D. Vanderbilt, New Journal of Physics 12, 053032 (2010).

[16] S. Zhong, J. E. Moore, and I. Souza, Phys. Rev. Lett. 116, 077201 (2016).

[17] A. Manchon, J. Železný, I. M. Miron, T. Jungwirth, J. Sinova, A. Thiaville, K. Garello, and P. Gambardella, Rev. Mod. Phys. 91, 035004 (2019).

[18] F. Freimuth, S. Blügel, and Y. Mokrousov, J. Phys.: Condens. matter 28, 316001 (2016).

[19] T. Qin, Q. Niu, and J. Shi, Phys. Rev. Lett. 107, 236601 (2011).

[20] D. Xiao, Y. Yao, Z. Fang, and Q. Niu, Phys. Rev. Lett. 97, 026603 (2006).

[21] N. R. Cooper, B. I. Halperin, and I. M. Ruzin, Phys. Rev. B 55, 2344 (1997).

[22] K. M. D. Hals and A. Brataas, Phys. Rev. B 91, 214401 (2015).

[23] F. Freimuth, S. Blügel, and Y. Mokrousov, Phys. Rev. B 90, 174423 (2014).

[24] F. Freimuth, S. Blügel, and Y. Mokrousov, Phys. Rev. B 92, 064415 (2015).

[25] C. Ciccarelli, K. M. D. Hals, A. Irvine, V. Novak, Y. Tserkovnyak, H. Kurebayashi, A. Brataas, and A. Ferguson, Nature nanotechnology 10, 50 (2014).

[26] O. Mosendz, J. E. Pearson, F. Y. Fradin, G. E. W. Bauer, S. D. Bader, and A. Hoffmann, Phys. Rev. Lett. 104, 046601 (2010).

[27] F. D. Czeschka, L. Dreher, M. S. Brandt, M. Weiler, M. Althammer, I.-M. Imort, G. Reiss, A. Thomas, W. Schoch, W. Limmer, et al., Phys. Rev. Lett. 107, 046601 (2011).

[28] D. Ralph and M. Stiles, Journal of Magnetism and Magnetic Materials 320, 1190 (2008).

[29] S. Nakatsuji, N. Kiyohara, and T. Higo, Nature 527, 212 (2015).

[30] I. Garate, K. Gilmore, M. D. Stiles, and A. H. MacDonald, Phys. Rev. B 79, 104416 (2009).

[31] E. van der Bijl and R. A. Duine, Phys. Rev. B 86, 094406 (2012).

[32] S. A. Yang, G. S. D. Beach, C. Knutson, D. Xiao, Q. Niu, M. Tsoi, and J. L. Erskine, Phys. Rev. Lett. 102, 067201 (2009).

[33] S. E. Barnes and S. Maekawa, Phys. Rev. Lett. 98, 246601 (2007).

[34] F. Freimuth, S. Blügel, and Y. Mokrousov, Phys. Rev. B 95, 094434 (2017).

[35] S. Y. Savrasov, Phys. Rev. Lett. 81, 2570 (1998).

[36] Y. Gao, D. Vanderbilt, and D. Xiao, Phys. Rev. B 97, 134423 (2018).

[37] C. Xiao and Q. Niu, Phys. Rev. B 101, 235430 (2020).

[38] F. Freimuth, S. Blügel, and Y. Mokrousov, Phys. Rev. B 96, 104418 (2017).

[39] T. Schulz, O. Alejos, E. Martinez, K. M. D. Hals, K. Garcia, L. Vila, K. Lee, R. Lo Conte, G. V. Karnad, S. Moretti, et al., Applied Physics Letters 107, 122405 (2015).

[40] E. Jué, C. K. Safeer, M. Drouard, A. Lopez, P. Balint, L. Buda-Prejbeanu, O. Boulle, S. Auffret, A. Schuhl, A. Manchon, et al., Nature materials 15, 272 (2016).

[41] C. A. Akosa, I. M. Miron, G. Gaudin, and A. Manchon, 
Phys. Rev. B 93, 214429 (2016). 